\title{
A summary of WIND magnetic clouds for years 1995-2003: model-fitted parameters, associated errors and classifications
}

\author{
R. P. Lepping ${ }^{1}$, D. B. Berdichevsky ${ }^{1,2}$, C.-C. Wu ${ }^{1,3}$, A. Szabo ${ }^{1}$, T. Narock ${ }^{1,2}$, F. Mariani ${ }^{4}$, A. J. Lazarus ${ }^{5}$, and \\ A. J. Quivers ${ }^{6}$ \\ ${ }^{1}$ Laboratory for Solar and Space Physics NASA-Goddard Space Flight Center Greenbelt, MD 20771, USA \\ ${ }^{2}$ L-3 Government Services, Inc., 1801 McCormick Dr., Suite 170, Largo, MD 20774, USA \\ ${ }^{3}$ University of Alabama in Huntsville, AL 35899, USA \\ ${ }^{4}$ Dipartimento di Fisica Universita degli Studi di Roma Tor Vergata 00133 Rome, Italy \\ ${ }^{5}$ Center for Space Research Mass. Institute of Technology Cambridge, MA 02139, USA \\ ${ }^{6}$ Woodlawn Magnet High School Woodlawn MD 21228, USA
}

Received: 6 May 2005 - Revised: 19 October 2005 - Accepted: 26 October 2005 - Published: 7 March 2006

\begin{abstract}
Interplanetary magnetic clouds (MCs) have been identified for the first 8.6 years of the WIND mission, and their magnetic field structures have been parameter-fitted by a static, force free, cylindrically-symmetric model (Lepping et al., 1990) with various levels of success. This paper summarizes various aspects of the results of the model fitting by providing: seven estimated model fit-parameter values for each of the $82 \mathrm{MCs}$ found, their objectively determined quality estimates, closest approach vectors (in two coordinate frames), fit-parameter errors for the cases of acceptable quality (50 cases, or $61 \%$ ), axial magnetic fluxes, axial current densities, and total axial current - as well as some examples of MC profiles for various conditions and "categories" for each case (e.g. $\mathrm{B}_{z}: \mathrm{N} \rightarrow \mathrm{S}$ or $\mathrm{S} \rightarrow \mathrm{N}$, etc.). MC quality is estimated from a quantitative consideration of a large set of parameters, such as the chi-squared of the model fit, degree of asymmetry of the B profile, and a comparison of two means of estimating radius. This set of MCs was initially identified by visual inspection of relevant field and plasma data. Each resulting MC candidate is then tested through the use of the MC parameter model, for various adjusted durations to determine the best fit, which helps to refine the boundarytimes. The resulting MC set is called Set 1. Another, larger, set (Set 2) of MCs is identified through an automated program whose criteria are based on general MC plasma and field characteristics at $1 \mathrm{AU}$ determined through past experience. Set 1 is almost fully contained within Set 2, whose frequency of occurrence better matches that of the sunspot cycle than Set 1 . The difference-set (Set 2-Set 1 ) is referred to as the magnetic cloud-like (MCL) set, whose members do not very well represent good flux ropes through modeling. We present a discussion of how a MC's front boundary is

Correspondence to: R. P. Lepping

(ronald.p.lepping@nasa.gov)
\end{abstract}

specifically identified in terms of multi-parameter considerations (i.e. any one or more of: increase in $\mathrm{B}$, directional discontinuity, magnetic hole in $\mathrm{B}$, drop in proton plasma beta, B-fluctuation level change, proton temperature drop, etc.), as well as through the application of the flux rope model. Also presented are examples of unusual MCs, as well as some commonly occurring relationships, such as the existence and frequency (approx. 1/2 the time) of upstream interplanetary shocks, and less frequent internal shocks.

Keywords. Interplanetary physics (Interplanetary magnetic fields; Solar wind plasma) - Solar physics, astrophysics and astronomy (Flares and mass ejections)

\section{Introduction}

This paper provides a comprehensive summary of the results of a WIND magnetic cloud (MC) study covering 8.6 years of interplanetary magnetic field (Lepping et al., 1995) and solar wind plasma (Ogilvie et al., 1995) observations, starting in early 1995. Except for a few cases, MCs are revealed to be generally large magnetic flux ropes (e.g. Priest, 1990; Gosling, 1990; Lepping et al., 1990; Burlaga, 1995; Kumar and Rust, 1996) in the solar wind, i.e. plasma embedded strong magnetic fields of approximately helical structure. A MC was originally defined empirically in terms of in-situ spacecraft measurements of magnetic fields and particles in the interplanetary medium, viz., it is a region in the solar wind having: 1) enhanced magnetic field strength, 2) a smooth change in field direction as observed by a spacecraft passing through the $\mathrm{MC}$, and 3) low proton temperature (and low proton plasma beta) compared to the ambient proton temperature (Burlaga et al., 1981; Klein and Burlaga, 1982; Burlaga, 1988, 1995). 

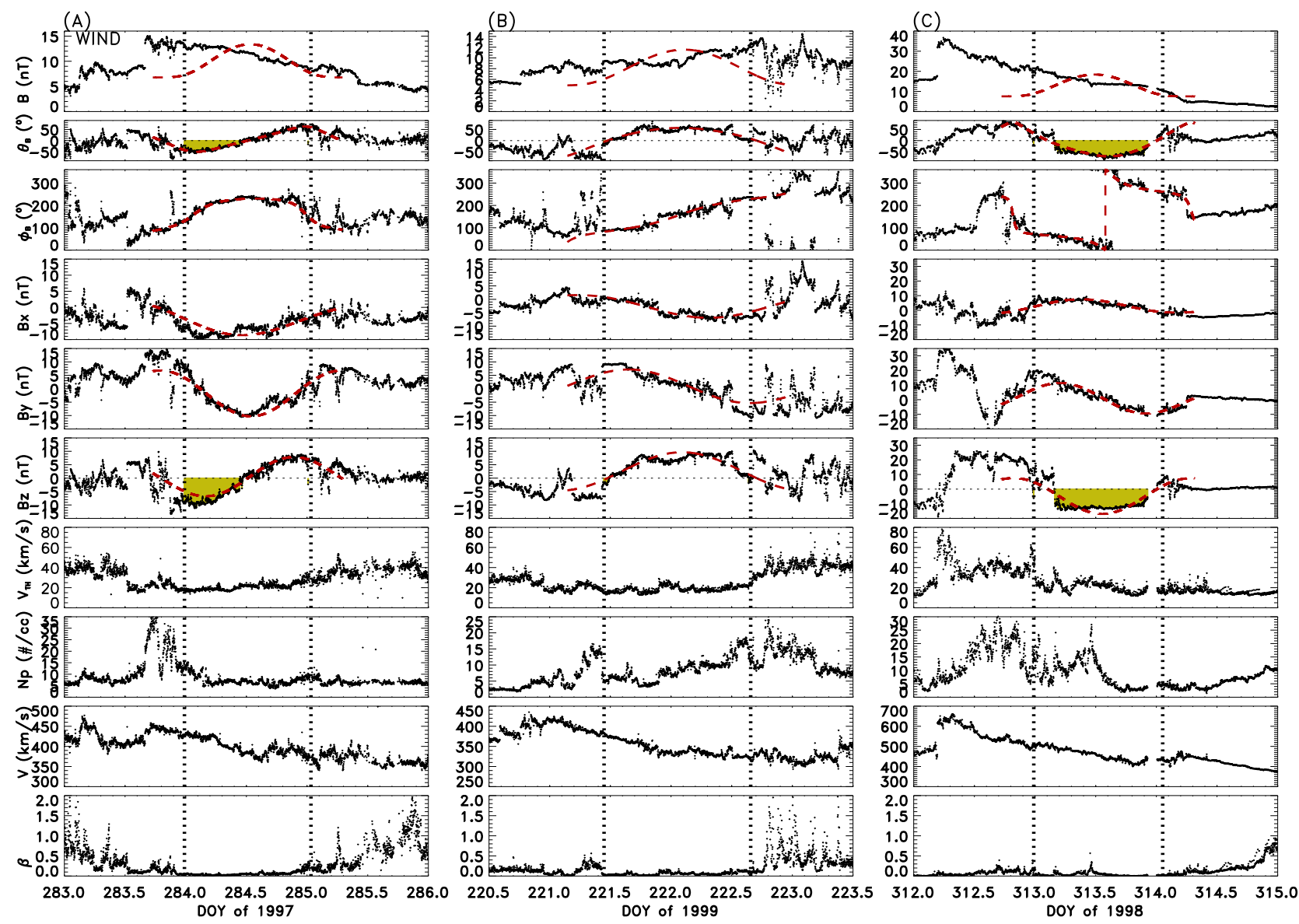

Fig. 1. Typical magnetic cloud (MC) field and plasma profiles in terms of magnetic field magnitude $(\mathbf{B})$, latitude $\left(\theta_{B}\right)$, and longitude $\left(\phi_{B}\right)$, $\mathrm{B}_{X, Y, Z}(\mathrm{GSE})$ components, thermal speed $\left(\mathrm{V}_{T h}\right)$, bulk speed $(\mathrm{V})$, proton number density $\left(\mathrm{N}_{P}\right)$, and proton plasma beta $\left(\beta_{P}\right)$ - in that order from top to bottom. The vertical lines indicate start and end times of the MC, and the (red) dashed curves are model-fits to observations, to be explained below. The regions shaded in yellow are where the observed $\mathrm{B}_{z}$ components are southward. (A) A case of a MC (starting on 10 October 1997 (Day 283) at 23.80 UT, with duration $\Delta \mathrm{T}$ of $25.0 \mathrm{~h}$ ) estimated to be closely aligned with the $\mathrm{Y}_{G S E}$-axis within about $2 \mathrm{E}$ (this is a $\rightarrow \mathrm{N}$ type, i.e. Category $11 \mathrm{MC}$ as described in Table 3), (B) another MC (starting on 9 August 1999 (Day 221) at 10:45 UT, with a duration of $29.0 \mathrm{~h}$ ) estimated to be strongly inclined (positively) with respect to the ecliptic plane by $76^{\circ}$, and finally a third (C) starting on 8 November 1998 \{Day 312\} at 23:45 UT, with a duration of 25.5 h) estimated to be strongly inclined (negatively) with respect to the ecliptic plane by $-71^{\circ}$. All three of these $\mathrm{MCs}$ are classified as $\mathrm{Q}_{0}=1$ cases, to be described below. Notice that case (A) has a uniform proton density, case (B) has an increase in density at the end of the MC, and case (C) has an increase in density in the early portion of the MC. This irregularity of density generally in MCs is the reason for not including density in the definition of a MC.

Magnetic clouds are also understood tacitly to be large structures, so that their durations are long, usually between about 7 and $48 \mathrm{~h}$ at $1 \mathrm{AU}$, averaging about $20 \mathrm{~h}$ in duration for the better examples; this feature is to be part of our definition of an interplanetary MC. MCs have been observed at distances other than at $1 \mathrm{AU}$, for example by the Helios (e.g. Bothmer and Schwenn, 1998) and Voyager spacecraft (e.g. Skoug et al., 2000; Burlaga et al., 2001). See Fig. 1 for examples of field and plasma profiles of fairly typical MCs at $1 \mathrm{AU}$ : (A) one (starting on 10 October 1997) whose axis is estimated to be closely aligned with the $\mathrm{Y}_{G S E}$-axis, (B) another (starting on 9 August 1999) estimated to be strongly inclined (positively) with respect to the ecliptic plane, and (C) another (starting on 8 November 1998) estimated to be strongly inclined (negatively) with respect to the ecliptic plane; in this context positive and negative indicate the polarity of the field along the MC axis where, for example, positive (negative) means that the direction of the axial field is "northward" ("southward") in GSE coordinates. All three cases satisfy the above definition of a MC but differ markedly with regard to the profiles of the latitude of the magnetic fields $\left(\theta_{B}\right.$ profiles), or $\mathrm{B}_{z}$ profiles. Notice that the proton density $\left(\mathrm{N}_{P}\right)$ profiles in the three examples of Fig. 1 differ markedly: the first (A) is uniform, the second (B) shows a distinct increase at the end, shown to be not uncommon for MCs (e.g. Burlaga et al., 1998; Lepping et al., 2003c), and the third (C) shows an enhancement in the early part of the structure. The fact of no apparent consistent pattern of the $\mathrm{N}_{P}$ profile within a $\mathrm{MC}$ is the reason that $\mathrm{N}_{P}$ was not chosen to be part of the definition of a MC (Burlaga, 1988). In some cases the $\mathrm{N}_{P}$ 
profile is very irregular, as we will see later. Figure 2 shows sketches of three examples of different flux rope orientations and their associated IMF $\mathrm{B}_{z}$ profiles in GSE coordinates. In this view the flux ropes are propagating toward the viewer. The one on the left corresponds to case (A) of Fig. 1 and the one in the center corresponds to example (C) of Fig. 1. Notice that all three examples in Fig. 2 have the same handedness, right-handed. (The red dashed curves shown in Fig. 1 (and in several other figures) are MC model parameter-fits to the magnetic field observations and will be explained below. They strictly hold only between the designated MC intervals, but are shown extended outside of them for comparison, generally showing significant deviation from the observations in one or more field components in the extended regions.)

MCs are among the largest transient structures in the interplanetary medium, but are smaller in size when compared to such very large solar wind structures as the immense heliospheric current sheet, co-rotating interaction regions (CIRs), and merged interaction regions (MIRs), all being quasi-periodic structures (see, e.g. Burlaga, 1995) at a $\approx 27$ day period in an Earth-fixed frame. However, MCs are so large that, even at $1 \mathrm{AU}$, it is very likely that they originate at the Sun, and many studies have confirmed that this is indeed the case (see, e.g. Burlaga, 1995; Larson et al., 1997; Marubashi, 1997; Berdichevsky et al., 2000, 2002; Webb et al., 2000); also see Farrugia (1997) on large-scale modeling of MCs in various possible field line topologies favoring the flux rope structure. Many, but not all, examples of MCs have been shown to be related to disappearing filaments (or solar erupting prominences as seen on the limb) on the Sun's surface (Bothmer and Schwenn, 1994). It has been suggested by Gosling (1990) that the initial stage of an interplanetary flux rope results from magnetic reconnection within rising, previously sheared, coronal magnetic loops, and Vrsnak et al. (1991) have shown analytically that prominence fields should have helical structure. And see Chen (1996) for a theoretical treatment associating expected erupting prominence conditions with a typically observed magnetic field profile of a MC at $1 \mathrm{AU}$.

There is a consensus developing that MCs are usually contained within interplanetary coronal mass ejections (ICMEs) (see, e.g. Gopalswamy et al., 1998), which are one form of solar transient events, sometimes known as solar ejecta. ICMEs are the interplanetary remnants of coronal mass ejections (CMEs); see a review by Schwenn (1996). An excellent study of solar ejecta signatures at $1 \mathrm{AU}$ was given by Goldstein et al. (1998). Other reviews on CMEs/ICMEs are by Gosling $(1990,2000)$, and more recently by Zurbuchen and Richardson (2006). Frequently used indicators of solar ejecta are average internal magnetic fields that are $\geq 2$ times the strength of the background field, steady orientated (or smoothly varying) fields over a relatively extended interval of time (12 to $48 \mathrm{~h}$ or so), plasma composition abnormalities, including enhanced plasma helium to hydrogen ratio (Hirshberg et al., 1972), abnormally low proton temperatures (Gosling et al., 1973), and energetic particle intensity depression (e.g. Barden, 1972; Cane et al., 1996). More detailed
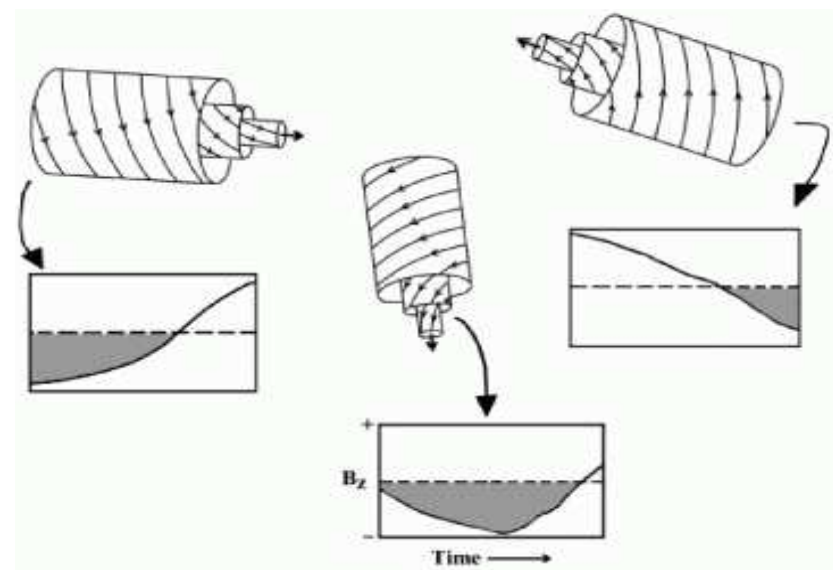

Fig. 2. The profile of the IMF $B_{z}$ within a MC can be understood in terms of the orientation of the MC as a flux rope. Here are sketches of three examples of different flux rope orientations and their associated IMF $\mathrm{B}_{z}$ (or $\theta_{B}$ ) profiles in GSE coordinates. The end-points of the boxes represent the boundaries of the MCs, and the shaded regions are where the $\mathrm{B}_{z}$ components are southward.

lists of indicators are given by Cane and Richardson (2003) and Berdichevsky et al. (2002). Abnormal plasma signatures and energetic particle shielding effects in the interplanetary medium are often more pronounced in MCs than in other less magnetically structured ejecta. The percentage of MCs contained within ICMEs apparently depends on specific conditions (e.g. Richardson and Cane (2006), who show a solar cycle dependence). But details on the relationship of MCs to ICMEs do not directly concern us here. For the most part we restrict our studies to properties of MCs per se and in some respects to their upstream shocks.

Roughly speaking, it is the reconfigurations of relatively strong, twisted, magnetic field-lines in restricted regions on the solar surface (or lower corona), with magnetic reconnection apparently participating (e.g. Antiochos and DeVore, 1999), that play a major role in the occasional expulsion of large flux rope structures and their related "cool" plasmas in the form of MCs. But the details of the solar birth and expulsion mechanisms of MCs/CMEs are still not agreed upon; see six proposed models for their origins listed by Rust (1999). However, because of the strong magnetic fields and low proton temperatures within them, MCs are regions of low (proton) plasma beta $\beta_{P}$ (where $\beta_{P}=$ thermal pressure/magnetic field pressure $\left.=2 \pi \mathrm{nkT}_{P} / \mathrm{B}^{2}\right)$. Often $\beta_{P}$ is very low $(\cong 0.12$ on average and closer to $\cong 0.08$ in the MC's central region) at $1 \mathrm{AU}$ (e.g. Lepping and Berdichevsky, 2000) and, hence, the dynamic behavior of MCs, at least out to $1 \mathrm{AU}$, and probably well beyond, is dominated primarily by their internal magnetic fields. Typically a MC's electron temperature is much higher than the proton temperature, and it is also usually not markedly different from the electron temperature of the normal solar wind. (But typically the electron and proton temperatures combined are not high enough to provide a sufficiently high thermal pressure to compete dynamically 
with the strong internal "field pressure" (Burlaga et al., 1981; Burlaga, 1995)). Therefore, the electron temperature is not as useful in helping to identify these structures. Hence, the net thermal pressure of a MC's internal plasma (from protons and electrons) is usually low compared to the magnetic field pressure, and after equilibrium is achieved the poloidal and toroidal magnetic field forces almost balance each other providing an approximately zero net force condition. This minimum energy state is referred to as "static force-free". In this state, electrical currents flow only along the magnetic field lines. This state is not unexpected for such structures in the solar wind, provided sufficient time passes from the time of their solar formation to the distant observing point/time for it to be achieved. Such a configuration theoretically yields a magnetic flux rope, which has a geometry that is almost always consistent (to a good approximation) with what has been ascertained from the actual magnetic field measurements in space at $1 \mathrm{AU}$ for most such events, i.e. those satisfying the (empirical) definition of a MC (Burlaga et al., 1981; Burlaga, 1988). Various suggested magnetic field configurations other than flux ropes, such as plasmoids, spheromaks, etc., have been introduced to account for these large regions of strong field intensity and "smoothly" changing field direction, but with much less success, as pointed out by Osherovich and Burlaga (1997).

Although there is not agreement as to the detailed nature of the solar source of a MC, it is likely that in its coronal stage (and probably even in earlier stages) it is in the form of a small flux rope, which then expands into a large flux rope of relatively cool protons, as it moves out into the interplanetary medium (Burlaga, 1995; Marubashi, 1997). But, of course, few MCs are pristine examples of flux ropes, and some considerably violate this structural form, because of various reasons, such as likely anisotropic birth conditions and subsequent expansion (common to most MCs), as well as due to their interactions with the surrounding plasmas. A MC expands until a near-equilibrium state with the surrounding pressure is established, usually beyond the distance of $1 \mathrm{AU}$ from the Sun. Evidence of MC expansion is seen clearly at $1 \mathrm{AU}$ by the observations of a (usually) slow decrease in bulk speed of the internal plasma as the MC passes the observing spacecraft; also see Gopalswamy et al. (2000) on the small degree of $\mathrm{MC}$ acceleration in the solar wind. Occasionally we observe a flat speed profile within a MC, suggesting that internal pressure equilibrium was established earlier than usual, somewhere between the Sun and Earth.

Lepping and Berdichevsky (2000) reviewed their past MC studies, using WIND data (for $\mathrm{N}=28$ cases of MCs, a much smaller sample than considered here) mainly for the solar "quiet" period, and compared the results with a MC study from an earlier "active period" of IMF data (spacecraft comprising mainly IMP-8 where there were $\mathrm{N}=18$ cases). In particular, they presented a preliminary summary and comparison of average MC characteristics for these two periods. Strictly speaking the spacecraft considered in the earlier study (solar active period) were: IMP's 1, 2, 5, 6, 7, and 8, ISEE 3, and Helios 1. This present study considerably updates the WIND part of the study, where $\mathrm{N}=82 \mathrm{MCs}$ are now considered, and presents several new associated parameters.

The goals of this paper are: 1) to provide a quantitative summary of the characteristics of the 82 MCs that have been parameter fitted by a modified version of the Lepping et al. (1990) MC fit program as applied to WIND data for the years 1995 to August 2003 (8.6 years), 2) to describe the modifications that have been added to the parameter fitprogram, 3) to describe the difficulties in identifying the boundaries of MCs, 4) to provide a scheme to quantitatively assess the "quality" of the model fitting, 5) to provide some average MC profiles as functions of various categories (e.g. by $\mathrm{B}_{z}$ orientation and flux rope handedness), 6) to estimate the errors on the fit-parameters for most of the $82 \mathrm{MCs}$, i.e. all but the ones considered of poor quality, to be quantitatively defined later, and finally 7) to briefly describe the results of a program that automatically identifies $\mathrm{MC}$ and magnetic cloud-like structures in the solar wind at $1 \mathrm{AU}$, with comparisons to actual MCs. Parts 4) and 5) address two of many ways of classifying MCs, i.e. by quality and field profile (mainly IMF- $B_{z}$ profile in this case), respectively. Recent modifications to our MC-fit program were made to provide the means of assessing objectively the quality of each fit. In the past subjective judgement of quality was made, guided only by a few quantitative measures, such as the value of a reduced $\chi^{2}$ of the fit, the degree of asymmetry, and whether or not the specific event appeared to satisfy cylindrical symmetry based on the expected global nature of MCs (to be clarified below). All of these measures are still applied, but others have been added, one based on estimating the size of the MC's cross-section in terms of the duration of the cloudpassage, and another based on considerations of the average field within the MC in "cloud" coordinates (defined below).

It is hoped that the information in this paper will be helpful to researchers in solar, interplanetary, and magnetospheric physics. In the past there have been many studies relating MCs to solar events, mostly with respect to timing or field chirality-matching, but with the extensive MC (i.e. interplanetary) results provided here, many more detailed connections should become available in relating a solar event to a specific MC. Also, it is well known that strong, usually long-lasting, negative IMF- $B_{z}$ fields (in GSE, or strictly GSM coordinates), among other quantities, play an important role in geomagnetic storm generation (e.g. Kamide et al., 1997). As first pointed out by Burlaga et al. (1981; also see Burlaga et al., 1990), a MC is a natural candidate for triggering of such a storm, because MCs always have intense internal fields (which is often also true for the region just ahead of the MC), and they almost always have extensive regions of strong negative $\mathrm{B}_{z}$ fields somewhere within their extent (see, e.g. Wilson (1990), Tsurutani and Gonzalez (1997), and Wu and Lepping (2002a,b (and references therein), 2005), Wu et al. (2004). Also see Burlaga et al. (1987), who discuss the importance of MCs in generating historically large magnetic storms, and Farrugia et al. (1997), who discuss the role of MCs in causing geomagnetic storms and various associated 
aspects, including how they facilitate the entry of solar energetic particles into the Earths magnetosphere. Webb et al. (2001) discuss the solar sources of both kinds of major geoeffective solar wind structures, i.e. transient, such as MCs and ICMEs, and recurring, such as corotating interaction regions.

\section{Magnetic cloud identification and parameter fitting model}

The MC fit-parameter values for a much smaller number of WIND MCs than are included here have appeared in an earlier publication in only a summary form (Lepping and Berdichevsky, 2000). These parameters (among others) are presented here in detail and for completeness.

2.1 Identifying magnetic clouds and their boundaries from plasma and field measurements

Shimazu and Marubashi (2000) have developed a means of detecting MCs in an automatic mode, which depends on certain characteristics of the variation of the magnetic field before checking the candidate structure with modeling. Their model also takes into consideration expansion of the MC. They find that flux ropes can be classified into two types, high and low density. In our studies we do not discriminate according to density, since we contend that it is an unreliable parameter for defining a MC (e.g. Lepping et. al., 2003c), and it was not part of the original empirical definition (Burlaga, 1988). Recently an automatic program providing a means of identifying MCs has been developed (Lepping et al., 2005) based on objective criteria following the empirical definition of a MC. Upon application it was recognized that this program provided what we refer to as many "magnetic cloud-like" structures (as well as both poor and high quality bona fide MCs, the quality of which was measured by the ability to fit them as flux ropes according to the model of Lepping et al., 1990). Hence, because of this, the automatic means of identification of MCs was not the means used to provide the more restricted set of MCs principally studied here; we say more on this in Sect. 6.

This section briefly discusses the usual difficulties in MC identification and in particular difficulty in ascertaining their exact boundary times; Sect. 6.0 extends discussion on MC identification in general. The plasma-field quantities commonly used in MC identification, and in particular MC boundary identifications, are proton plasma beta $\left(\beta_{\mathrm{p}}\right)$, proton temperature $\left(\mathrm{T}_{\mathrm{P}}\right)$, central or average speed $(\mathrm{V})$, interplanetary magnetic field (IMF) intensity, IMF-longitude and latitude angles, and sometimes the speed profile. In particular, the front boundary is easily determined if a directional discontinuity occurs in the magnetic field. This is also true for the rear boundary, but less commonly. Magnetic "holes" may appear in the B-profile at the boundaries, as pointed out by Burlaga (1995) (also see Farrugia et al. (2000), who used the presence of magnetic holes at the boundaries of the

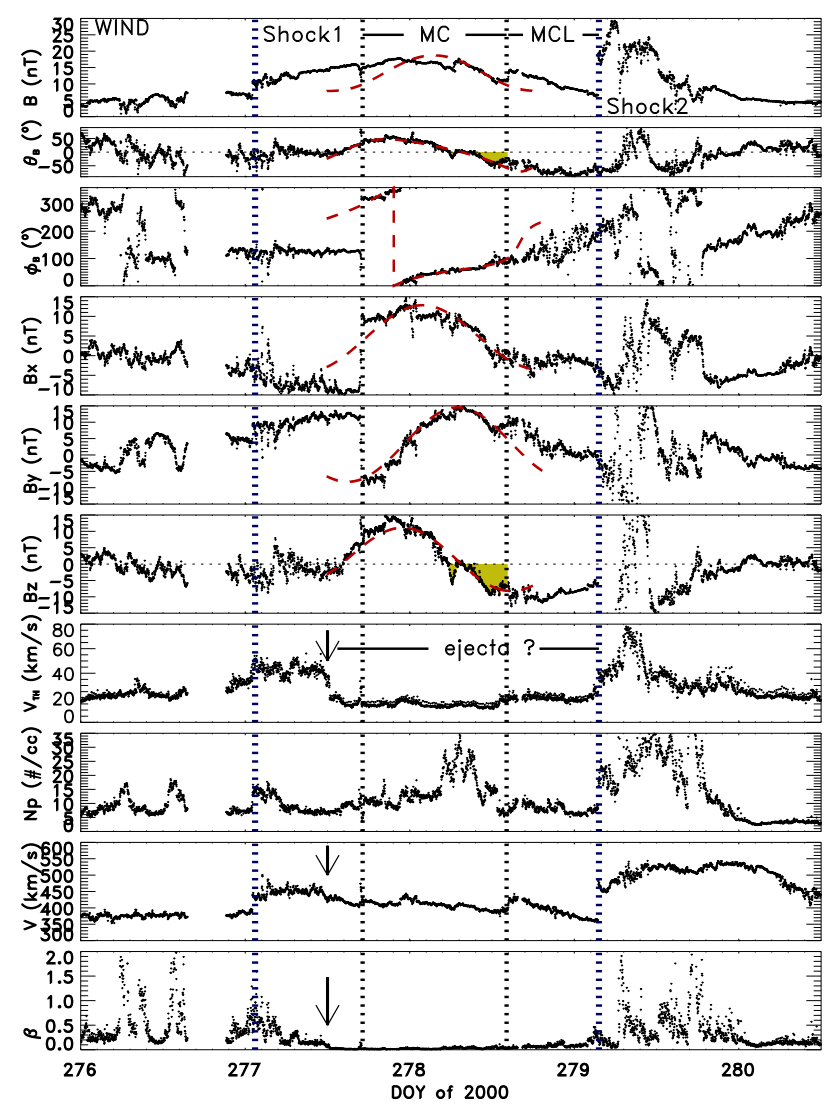

Fig. 3. An example of a MC starting on 3 October (Day 277), 2000 at hour 17.1 given in the same format as that of Fig. 1, where the magnetic field profile (and related (red dashed) model fitting curves) seems to demand one set of MC-boundaries, and the plasma quantities, especially proton temperature and proton plasma beta, require a slightly different set. We point out two forward shocks (shock1 and later shock2). The vertical arrows (on three of the bottom four panels) at about mid-day of 3 October indicate where $\mathrm{V}_{\mathrm{Th}}, \beta$, and $\mathrm{V}$ show noticeable change; in the case of $\mathrm{V}$ it is where the speed starts to drop smoothly, and somewhat slowly, at least until the start of the MC. From the time of the arrows to near shock 2 may be a region of ejected material (as denoted), delineated best by $\mathrm{V}_{\mathrm{Th}}$, and whose earliest part reaches outside of the front of the MC. MCL refers to a magnetic cloud-like region. The region shaded in yellow is where the observed $\mathrm{B}_{z}$ component is southward.

"Christmas 1996" MC, to help in its identification). The field and plasma profiles are not always in agreement with regard to boundary identification, especially when considering the basic magnetic field profile based on the fundamental definition of a MC, on the one hand, and proton plasma beta and/or proton temperature, on the other. And we must stress that the model itself is used to help constrain the estimated location of the MC boundaries, in the sense that the full set of magnetic field samples (in the form of reasonably chosen averages) throughout the MC participates in choosing the MC interval, along with the resulting model parameter-fit "quality" (defined below). In Fig. 3, we show an example of a MC (starting on 3 October 2000 at hour 17.1 and lasting $21.1 \mathrm{~h}$ ) 


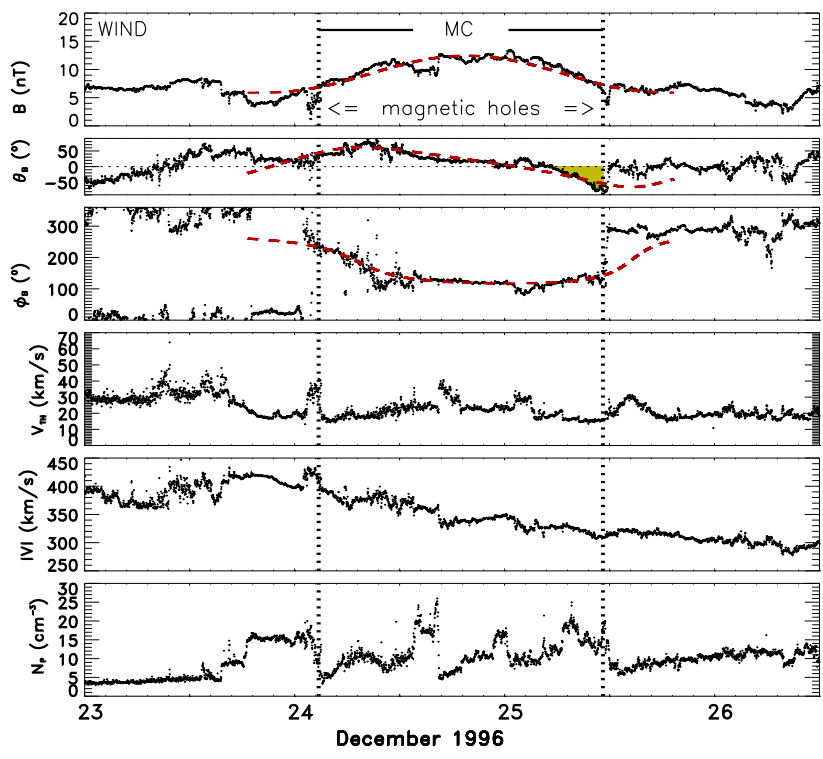

Fig. 4. Magnetic field and plasma profiles in the same format as that of Fig. 1 (but without the $\mathrm{B}_{X, Y, Z}$ components) for the case of the 24 December 1996 MC, where again the dashed curves are model-fits. Especially noticeable are: the nearly symmetrical magnetic field intensity within the MC, the magnetic holes at the MC boundaries (seen in the B-profile), and the irregular patterns in thermal speed and density. For example, the density profile differs dramatically from those in A and B of Fig. 1, and is somewhat different from that in $\mathrm{C}$ of that figure. Notice that this $\mathrm{MC}$ is in the $\mathrm{N} \rightarrow \mathrm{S}$ Category (in contrast to the MC in Fig. 1A which is a $\mathrm{S} \rightarrow \mathrm{N}$ type - see Table 3). For an $\mathrm{N} \rightarrow \mathrm{S}$ type there is the possibility of predicting the point where $B_{Z}$ is min. (usually a southward field), based on the early part of the MC, for $D_{s t}$ forecasting. Holes (when they exist) and directional discontinuities are very helpful in choosing the proper $\mathrm{MC}$ boundaries. The brief region shaded in yellow is where the observed $\mathrm{B}_{z}$ component is southward.

where the field seems to demand one set of boundaries and the plasma quantities another, especially at the front boundary. The MC's front boundary is probably well determined, because of the sharp directional discontinuity in the field occurring there. This situation might arise because the $\mathrm{MC}$ is either or both expanding and interacting with the surrounding plasma, and we stress that we are presently using a static model, so expansion is not yet considered. Other inadequacies of the MC model used are also apparently responsible for such incompatibilities; the model is highly idealized, as we will see. It is interesting that in the case of the 3 October $2000 \mathrm{MC}$ there is modest expansion, as seen by the very gradual drop in the speed V (which is somewhat irregular compared to most expanding MCs), but there is not a very strong front-side interaction in this case, as indicated by the absence of field compression in the early part of the MC. But notice that there is a driven shock (shock1) just after the start of 3 October (DOY $=277$ ) at about $16 \mathrm{~h}$ before the start of the MC. We point out another forward shock (shock2) following the MC. Between the end of the MC and this second shock there appears to be another possible MC (labeled MCL here), but after attempted MC fitting it does not show a good force free flux rope structure, so we refer to it as magnetic cloudlike. From the time of the vertical arrows (in three of the bottom four panels) to the second shock the proton thermal speed $\left(\mathrm{V}_{\mathrm{Th}}\right)$ is low, typical for MCs but also typical for ejecta generally (and for ICMEs). Hence, a possible interpretation for this region, besides those given above, may be that simply two MCs exist within a larger region of ejecta.

In Fig. 4 we show magnetic field and plasma profiles for the MC starting on 24 December 1996, in order to make a few important points. First, the field intensity B is nearly symmetrical within the MC, which is somewhat unusual (compare to typical Bs seen in Fig. 1), and, second, magnetic "holes" are seen at the MC boundaries in the B-profile, a not uncommon feature of MCs. Third, the speed (V) profile, although showing a distinct gradient, is not smoothly changing in this case, which contrasts to uniformly dropping speed profiles commonly seen within most MCs, as we saw in examples B and C of Fig. 1. Fourth, the V-gradient is of only moderate strength, which is consistent with the $\mathrm{MC}$ having a symmetric B-profile, in the sense that B is usually distorted to more intense values in the front of many MCs (see examples $\mathrm{A}$ and $\mathrm{C}$ in Fig. 1). This occurs when the plasma is relatively fast in the MC's front with respect to upstream solar wind speeds. Fifth, typically this speed-differential (i.e. the speed between the MC's front and the upstream speed) appears to play a more prominent role in the MC's B-profile distortion (from a temporally-symmetric profile) than the effect due to MC expansion (Lepping et al., 2002). Expansion is responsible for shifting the static model's central peak in B to earlier times (e.g. Osherovich, et al., 1993b). Hence, most MCs have higher B values in their fronts than in the central or rear regions.

\subsection{The cloud-fitting model and recent modifications}

As mentioned, MCs at $1 \mathrm{AU}$ are approximately force-free structures (Goldstein, 1983; Marubashi, 1986; Burlaga, 1995). The MC's geometry is ideally that of helical magnetic field (B) lines confined to a flux tube, which is curved on a scale of about $1 \mathrm{AU}$ at $1 \mathrm{AU}$ when considered globally (see bottom of Fig. 5). When examined locally, the structure is approximately cylindrically symmetric, and the pitch angle of the helical field lines increases with increasing distance from the axis of the MC, such that the field is aligned with the axis of symmetry at the position of the axis and perpendicular to it on the MC's boundary. See the top of Fig. 5 (top) where a spacecraft is shown moving through the local representation of the MC at some oblique angle. This is to be envisioned in the context of the ideal representation of the global MC (Fig. 5, bottom). A useful analytical approximation for this field configuration is the static, constant-alpha, force-free, cylindrically symmetric configuration (Burlaga, 1988), given by the Lundquist solution of

$\nabla^{2} \mathbf{B}=-\alpha^{2} \mathbf{B}$, 


\section{Magnetic Cloud}

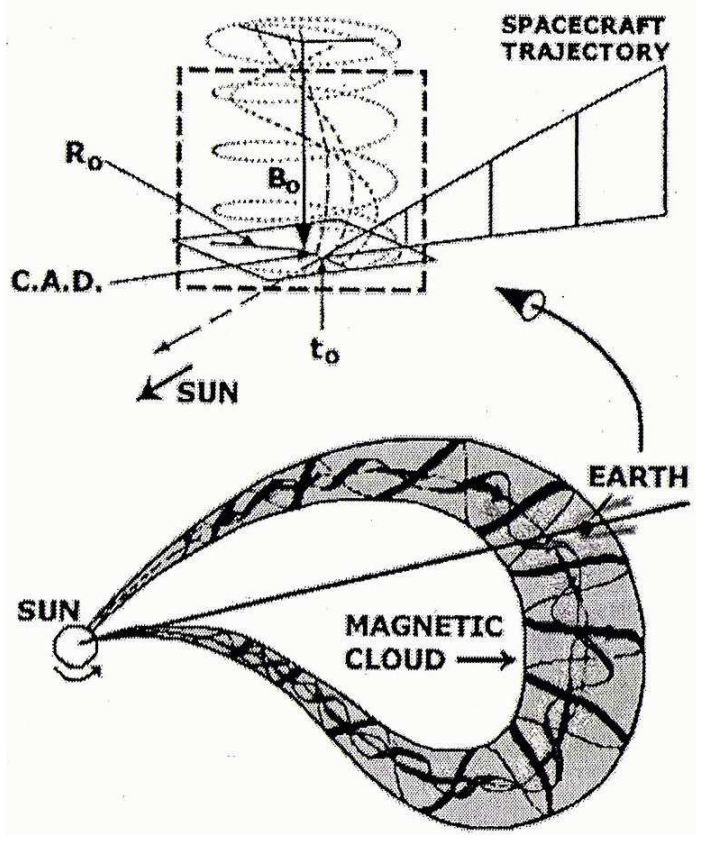

Fig. 5. (Top) A sketch of a cylindrically symmetric flux rope representing a MC locally, relative to the observing spacecrafts trajectory through it. Four model fit-parameters $\left(\mathrm{R}_{0}, \mathrm{~B}_{0}, \mathrm{t}_{0}\right.$, and closest approach distance (C.A.D.)) of the total of seven are shown; $\phi_{\mathrm{A}}, \theta_{\mathrm{A}}$, (the MCs axial longitude and latitude, respectively) are two others, and handedness $(\mathrm{H})$ is the last. In this case, if the thumb of the right hand is along the $\mathbf{B}_{0}$-direction and the fingers of that hand are parallel to the direction of the tangential field at the boundary, then the MCs handedness is right-handed. If it took the left hand to satisfy these alignments with the fields, the MC is left-handed. (Bottom) A global representation of a MC and its relationship to the local spacecraft passage (after Marabashi, 1997; also see Burlaga et al., 1990).

which results from assuming $\mathbf{J}=\alpha \mathbf{B}$ and the use of Maxwell's equations (Lundquist, 1950), which we express in the International System (SI) of units. More accurate MC models have considered the possibility that MCs expand as they move away from the Sun (Burlaga et al., 1981; Farrugia et al., 1992; Osherovich et al., 1993b, 1995; Marubashi, 1997; Berdichevsky et al., 2003) and/or the possibility of a violation of cylindrical symmetry (Lepping et al., 1998; Vandas et al., 2005a). We fit the Lundquist (1950) solution of Eq. (1) to averages of $\mathbf{B}$ (in GSE coordinates) using a modified method of Lepping et al. (1990). The Lundquist (Bessel function) solution is:

$B_{A}($ axial $)=B_{0} J_{0}(\alpha r), \quad B_{T}($ tangential $)=B_{0} H J_{1}(\alpha r)$,

and $B_{R}($ radial $)=0$,

where for any given $\mathrm{MC}$ the values for $\mathrm{B}_{0}, \alpha$, and $\mathrm{H}$ are to be determined, along with four other parameters described below. See Fig. 6 (left) for a representation of the Bessel function curves and how the full vectors are envisioned (right of the figure) in the typical case, where the radius is $R_{0}$ (single

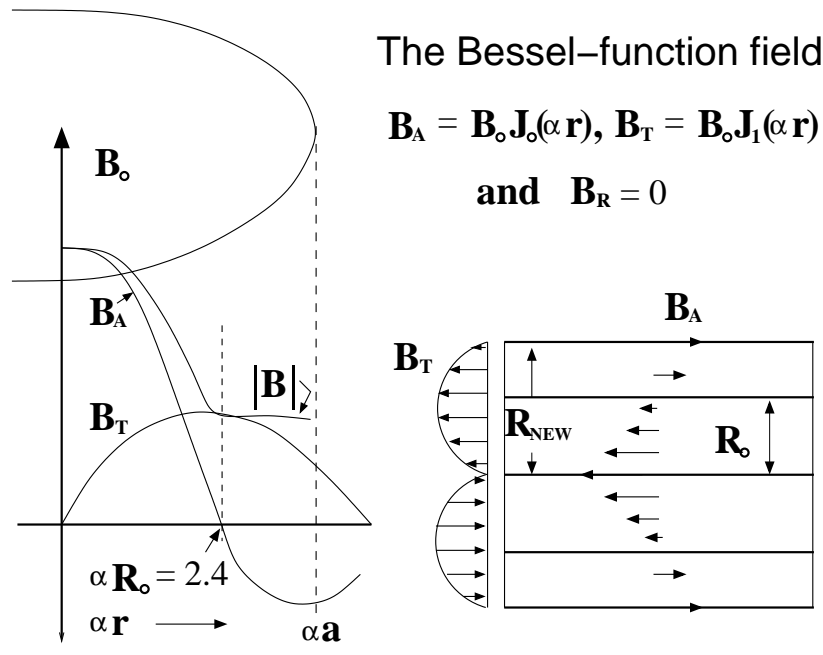

Fig. 6. (Left) The Bessel function curves resulting from the Lepping et al. (1990) model and (right) a sketch of the resulting vector fields. $\mathrm{B}_{\mathrm{A}}$ is the axial field, $\mathrm{B}_{\mathrm{T}}$ is the tangential field, and $\mathrm{B}_{\mathrm{R}}=0$ is the radial field (with respect to the flux ropes axis). The $\mathrm{B}_{\mathrm{T}}$ field (shown in the plane of the figure on the right) is in actuality normal to it. Normally the MCs radius is determined to be $\mathrm{R}_{0}$, but occasionally is shown to be $\mathrm{R}_{\mathrm{NEW}}$ (see text).

axial polarity), and in the special case where the MC possesses dual axial polarity, where the outer radius is given as $\mathrm{R}_{\mathrm{NEW}}$. That is, on (relatively rare) occasions we see examples of flux ropes with a core-annulus structure, where the inner radius, $\mathrm{R}_{0}$, separates the core from the annulus; this will be exemplified below. For most cases a MC has only a core with radius $\mathrm{R}_{0}$. The scheme was applied to 82 identified cases of MCs observed in the WIND data in the form of $15 \mathrm{~min}, 30 \mathrm{~min}$, or $1 \mathrm{~h}$ averages, depending on the duration of the specific event, such that there was an attempt to keep the number of points in the range of $\mathrm{N}$ : 20-90, but typically $\mathrm{N}=40$. For example, a $\mathrm{MC}$ of $40 \mathrm{~h}$ duration would require 1 - $\mathrm{h}$ averages for the fitting, and a $\mathrm{MC}$ of $\approx 10 \mathrm{~h} \mathrm{du}-$ ration would require 15 -min averages, but 30-min averages were used most often. In the scheme the least-squares fitting is initially carried out using unit normalized magnetic field data. That is, only the field's direction is considered at first. (A simple linear scaling of the model fields magnitude to the observed fields magnitude is done after the leastsquares fitting, as a final step.) A "reduced" chi-squared deviation to the fit, $\chi^{2} /(3 \mathrm{~N}-\mathrm{n})\left(\equiv \chi_{R}^{2}\right)$, where $\mathrm{N}$ is the number of time-averaged points and $n=5$ is the number of parameters in this part of the fitting, is calculated to measure the quality of the fit. The chi-squared quantity parameter is dimensionless since the magnetic field was unit normalized up to this point; actually $\left\{\chi^{2} /(3 \mathrm{~N}-\mathrm{n})\right\}^{1 / 2}$ (i.e. $\left.\left|\chi_{R}\right|\right)$ is displayed. The full set of 7 fitted parameters are:

- $\mathrm{B}_{0}$, the axial field intensity;

- $\mathrm{H}$, the handedness of the field twist, right-handed $(\mathrm{H}=+1)$ or left-handed $(\mathrm{H}=-1)$; 
- $\mathrm{R}_{0}$, the radius of the $\mathrm{MC}$;

- $\phi_{A}, \theta_{A}$, the longitude and latitude, respectively, of the MC's axis (GSE coordinates);

- $\mathrm{t}_{0}$, the center time (i.e. the closest approach time); and

- $\mathrm{Y}_{0}$, the closest approach (CA) distance (often called the impact parameter) and generally given below as a percentage of $\mathrm{R}_{0}$. (In that case, $\mathrm{CA} \equiv 100 \mathrm{Y}_{0} / \mathrm{R}_{0} \%$.)

The last five parameters are the $n=5$ considered in the reduced chi-squared fit process. Note that we choose the (normal, i.e. core) boundary of the MC such that the magnetic field becomes purely azimuthal there, i.e. where $r=R_{0}$; see Fig. 6. Hence, the argument of the Bessel functions at that point is $\alpha \mathrm{r}=2.40$ (i.e. $\mathrm{R}_{0}=2.40 / \alpha$ at the core boundary), to three place accuracy. However, the exact end-points are not always evident in the data, and sometimes many trial-fits are necessary. Figure 5 shows explicitly some of these fitparameters. See Appendix A of Lepping et al. (2003a) for details on how the MC parameter fitting technique is carried out. We refer to the analysis interval chosen for the fitting procedure as the "duration" [ $\Delta \mathrm{T}=($ end time - start time $)]$ of MC passage, which is not necessarily equal to twice the models estimated center time, $\mathrm{t}_{0}$. By allowing $\mathrm{t}_{0}$ to be a free parameter we are giving the model a chance to tell us a best estimate of the center time even in cases where $\Delta \mathrm{T} / 2$ may not have been well chosen. In many cases, however, any natural distortion of the $\mathrm{MC}$ will cause $\mathrm{t}_{0}$ and $\Delta \mathrm{T} / 2$ to differ.

It is convenient to define a so-called cone angle $\left(\beta_{C A}\right)$, defined as the angle between the MCs axis and the Sun-Earth line (specifically the $\mathbf{X}_{G S E}$-axis, positive toward the Sun, as usual), i.e.

$\cos \beta_{C A}=\cos \theta_{A} \cos \phi_{A}$.

Now we derive the formulae used to estimate the axial current density $\left(\mathrm{J}_{0}\right)$ and the axial magnetic flux $\left(\Phi_{0}\right)$ according to the MC model used here (Lepping et al., 1990). Since $\mathbf{J}=\alpha \mathbf{B}$, then the axial current density, $\mathbf{J}_{0}=\alpha \mathbf{B}_{0}$, and therefore, $\mathrm{J}_{0}=2.40 \mathrm{~B}_{0} / \mathrm{R}_{0}$,

according to our choice of boundary, $\alpha=2.40 / \mathrm{R}_{0}$. Since $\mathrm{B}_{0}$ and $R_{0}$ are quantities that are estimated directly by the field data fitting process, $\mathrm{J}_{0}$ is a direct by-product of the fitting process. The axial magnetic flux is defined as

$$
\begin{aligned}
\Phi_{0} & =\text { Flux }=\int \mathbf{B} \cdot d \mathbf{S}=\int B_{A} d S=B_{0} \int J_{0}(\alpha r) 2 \pi r d r \\
& =\left(2 \pi B_{O} / \alpha^{2}\right) \int J_{0}(x) x d x,
\end{aligned}
$$

over $\mathrm{x}$ : $0-2.40$, where $\mathrm{x} \equiv \alpha \mathrm{r}$ and $\mathrm{x}_{0}=\alpha \mathrm{R}_{0}=2.40$, at the MC's boundary according to the model, and where Eq. (2), for the axial field component (in terms of the zeroth-order Bessel function, $\left.J_{0}\right)$, was employed. Hence,

$\Phi_{0}=1.36 B_{0} R_{0}^{2}$,

again where $B_{0}$ and $R_{0}$ are quantities that are estimated directly. Obviously Eq. (6) does not hold for core-annulus cases, if one desires the net axial flux.

\subsection{Recent modifications to the model}

Recent modifications were added to the MC fit-model, to help us better judge the quality of the fitting. One of these is the use of the quantity check (where the closer its value gets to zero the better), and is defined as

check $=\left(\mathrm{R}_{\Delta \mathrm{T}}-\mathrm{R}_{0}\right) / \mathrm{R}_{0}$

where

$R_{\Delta T}=\sqrt{\left[Y_{O}^{2}+\left(\sin \beta_{C A} V_{C} \Delta T / 2\right)^{2}\right]}$,

and where $\Delta \mathrm{T}$ is the observed duration of MC-passage, $\mathrm{V}_{C}$ is the center speed of the $\mathrm{MC}$ (being close to the average speed across the MC) and taken at the $1 / 2$-duration point, $\beta_{C A}$ is the angle between the clouds axis and the Sun-Earth line, given by Eq. (3), and $\mathrm{Y}_{0}$ is the closest approach distance. That is, the value of the quantity "check" tests for consistency between two different means of obtaining estimates of the MC's radius: 1) directly from the models $\mathrm{R}_{0}$ and 2 ) from $\mathrm{R}_{\Delta T}$, which is based on the MC's speed and duration, and where we must account for the tilt of the MC's axis (via sin $\left.\beta_{C A}\right)$ and for the fact that the spacecrafts closest approach is usually not zero (via $\mathrm{Y}_{0}$ ). Other useful quantities are:

asf $=\left|\left(1-2 t_{0} / \Delta T\right)\right| \times 100 \%$,

called the "asymmetry factor" (where $0 \%$ is perfect symmetry), and consideration of the average field components (taken across the $\mathrm{MC}$ ) in cloud coordinates, $<\mathrm{B}_{\mathrm{X}}>_{\mathrm{Cl}}$, $<\mathrm{B}_{Y}>_{C l},<\mathrm{B}_{\mathrm{Z}}>_{C l}$ (as described in Appendix A). The following URL, within the WIND/MFI Website, explains the basis for the $\mathrm{Cl}$ coordinates and provides a matrix that will transform from GSE coordinates to $\mathrm{Cl}$ coordinates: http: //lepmfi.gsfc.nasa.gov/mfi/ecliptic.html.

In brief, in $\mathrm{Cl}$ coordinates the $\mathbf{X}_{\mathrm{Cl}}$-axis is along the MC's axis, positive in the direction of the positive polarity of the axial field, the $\mathbf{Z}_{C l}$-axis is the projection of the trajectory of the spacecraft [relative to the MC's velocity (essentially the $\mathbf{X}_{G S E}$-axis)] onto the cross-section of the $\mathrm{MC}$, and $\mathbf{Y}_{C l}=\mathbf{Z}_{C l} \times \mathbf{X}_{C l}$. Ideally then, $<\mathrm{B}_{X}>_{C l}$ should be always positive and $\left\langle\mathrm{B}_{Y}\right\rangle_{\mathrm{Cl}}$ should be zero, because of the fundamental field structure of the force free model (Eq. 1) and this definition of the $\mathrm{Cl}$ coordinate system. However, the expectation that $\left\langle\mathrm{B}_{X}>_{C l}\right.$ should be always positive is not expected to hold for the special "core-annulus" cases, as mentioned in Sect. 2.2. (Notice that the $\mathbf{Y}_{\mathrm{Cl}}$-axis is aligned with a line that passes through the closest approach point and the MC's axis. We often refer to this as the closest approach axis.) These considerations will enter into evaluation of the model fits quality $\left(\mathrm{Q}_{0}\right)$, along with some other fundamental aspects of the fit, discussed below in Sect. 3.0.

Finally, in the MC parameter least-squares fitting procedure it is imperative to provide flags to warn us of: 1) lack of convergence (or at least poor convergence), denoted by the F-flag, and 2) the inability to accurately estimate MC handedness $(\mathrm{H})$, indicated by the f-flag. In particular, the f-flag depends on examination of the sign of the $\mathrm{Y}_{\mathrm{Cl}}$-component 
of the average magnetic field of the $\mathrm{MC}$ in $\mathrm{Cl}$ coordinates, separately for the first and second halves of the $\mathrm{MC}$, where the half-way point is assumed equal to the mid-point of the estimated duration. From simple symmetry the average $\mathrm{Y}_{\mathrm{Cl}^{-}}$ field component in these two halves of the MC should be of opposite sign (and ideally of equal magnitude), and this signphasing is independent of which side of the $\mathrm{MC}$ the observer passes first. When these two average fields are shown, in fact, to have opposite signs, the f-flag is set equal to "OK" (no error), but if they are of the same sign, the f-flag is set equal to "NOT OK" (giving a warning). [As we will see, 6 out of $82 \mathrm{MCs}$ (i.e. 7\%) will receive this warning.] Note that, according to our MC model, the axial field component $\left(\mathrm{B}_{X, C l}\right)$ is always positive in the $\mathrm{Cl}$ coordinate system allowing us to ascertain $\mathrm{H}$ from $\mathrm{B}_{Y, C l}$ alone.

Concerning the F-flag, in the least-squares iteration process of fitting the field of the MC we step-wise adjust the direction of the MCs axis by $\Delta \theta_{\mathrm{i}}$ (change of axial latitude) and $\Delta \phi_{\mathrm{i}}$ (change of axial longitude), finally taking note of the size of these changes for the last iteration (see Appendix A of Lepping et al., 2003a). Usually these final angle-changes are very small, such as a few degrees, indicating proper convergence, but we arbitrarily allow angle-changes as large as $10^{\circ}$. If the last iteration required a change larger than $10^{\circ}$ for either angle, we designate the process as non-convergent, and the program shows an F-flag="NOT OK". [As we will see, 3 of the $82 \mathrm{MCs}$ will receive NOT OK designations (i.e. $4 \%$ ) for the F-flag.]

\section{Means of judging quality and some peculiarities}

The quantities that are chosen for estimating the "quality" (designated $\mathrm{Q}_{0}$ ) of $\mathrm{MC}$ model parameter fitting are: Diameter $\left(\equiv 2 \mathrm{R}_{0}\right), \chi_{R}\left(\equiv \sqrt{\left(\chi_{R}^{2}\right)}\right), \mathrm{CA}\left(\equiv Y_{0} / R_{0}\right)$, asf, check, $<\mathrm{B}_{X}>_{C l},<\mathrm{B}_{Y}>_{C l},<\mathrm{B}_{Z}>_{C l}$, the f-flag (for ability to determine handedness: OK or NOT OK), the F-flag (for convergence: OK or NOT OK), and the cone angle $\left(\beta_{C A}\right)$. $\mathrm{Q}_{0}$ can take on the values of $1,2,3$, where 1 is good and 3 is poor. By quality we mean a measure of the MC models ability to fit the magnetic field data for any given event period, as well as to satisfy some important consistency constraints. For example, one such constraint is that the two different kinds of estimates of the flux rope radius agree, i.e. that $\mid$ check| is small (see Eq. 7). Specifically, the $\mathrm{Q}_{0}$ value is assigned according the criteria based on magnetic field quantities, determined empirically and described in Appendix A. In Sect. 6, in another context, we extend our concept of MC quality (through automatic identifications of MCs) by including plasma quantities, i.e. proton temperature and proton plasma beta. As mentioned above, we often tried various types of field averages in the model fitting. The results of these various attempted fittings for a given MC were compared, for some trial runs, to see if a particular type of average provided higher quality fits than the others, in which case the highest quality case would be the trial that was accepted.

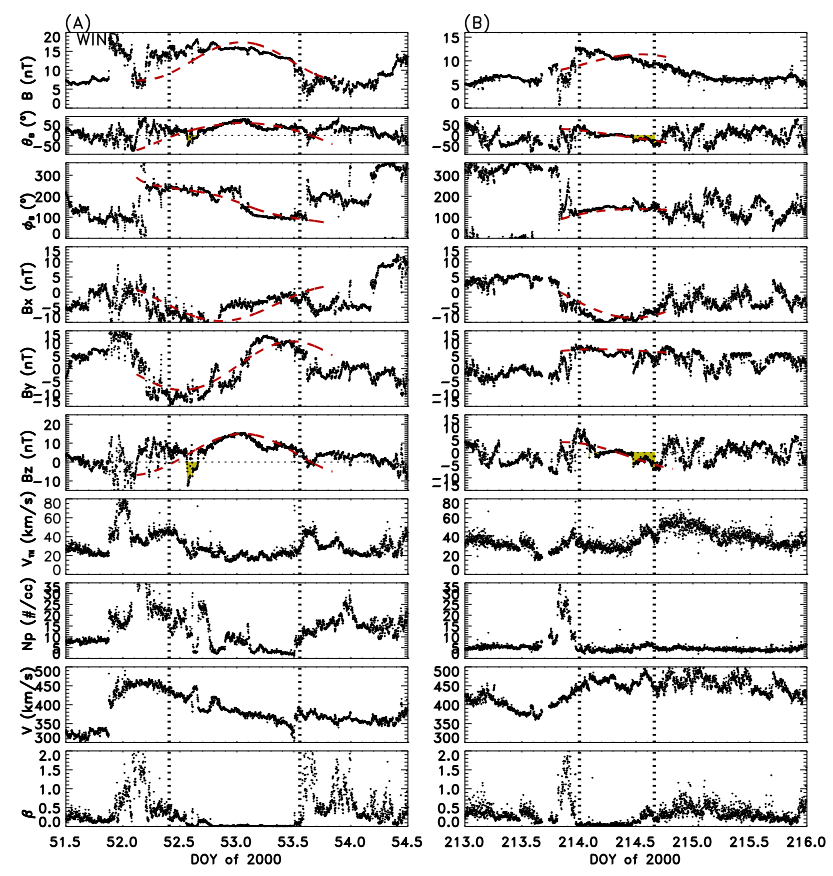

Fig. 7. Two examples of poor quality $\left(\mathrm{Q}_{0}=3\right) \mathrm{MCs}$, both from the year 2000: (A) the event of 21-22 February Days 52 and 53 and (B) that of 1 August Day 214, presented in the same format as that of Fig. 1. In the first one (A) the quality is poor, because of the poor parameter fit, where $\chi_{R}$ was high, 0.215 , and in the second one (B) $\chi_{R}$ was acceptable (0.122), but the asymmetry factor (asf)

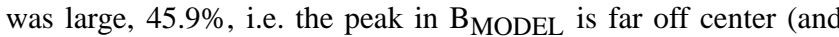
notice that there was little change in the latitude of the field within this MC making MC parameter fitting difficult). The regions shaded in yellow in both cases are where the observed $\mathrm{B}_{z}$ components are briefly southward.

In Fig. 7 we show two examples of $\mathrm{Q}_{0}=3 \mathrm{MCs}$. For the event in Fig. 7A, that of 21-22 February 2000, the quality is poor, because of the poor parameter fitting, i.e. where $\chi_{R}$ was high $(0.223)$. Also, although not related to quality, $\theta_{A}$ was unusually large, $75^{\circ}$, in this case. In Fig. $7 \mathrm{~B}$, showing the MC of 1 August 2000, the $\chi_{R}$ was acceptable (0.121), but the asf was large $(60.9 \%)$. Both MCs had moderate length durations, 27.5 and $15.9 \mathrm{~h}$, respectively. Notice that the $\theta_{B}$ curve of Fig. 7B varies little across the MC indicating that the observing spacecraft was quite distant from the MCs axis at time of closest approach agreeing with our estimate of $\mathrm{CA}=-82 \%$ for this case. By comparison with these low quality cases (where $\mathrm{Q}_{0}=3$ ), all three examples in Fig. 1 are $\mathrm{Q}_{0}=1$ cases, where we see that the parameter fittings and symmetries are rather good.

We have shown that $\mathrm{Q}_{0}$ is not strongly dominated by any one of the values of its input quantities. Quality's strongest dependence, however, is on asf (correlation coefficient (c.c.) of 0.42) with the criteria related to $\left\langle\mathrm{B}_{Y}\right\rangle_{C l}$ (for separating $\mathrm{Q}_{0}=1$ from $\mathrm{Q}_{0}=2$ cases), $\left\langle\mathrm{B}_{X}\right\rangle_{C l}, \chi_{R}$, |check $\mid$, $\beta_{C A}$, and $|\mathrm{CA}|$ being of intermediate importance; they have |c.c.|s from only 0.35 to 0.22 , dropping in the order shown. 

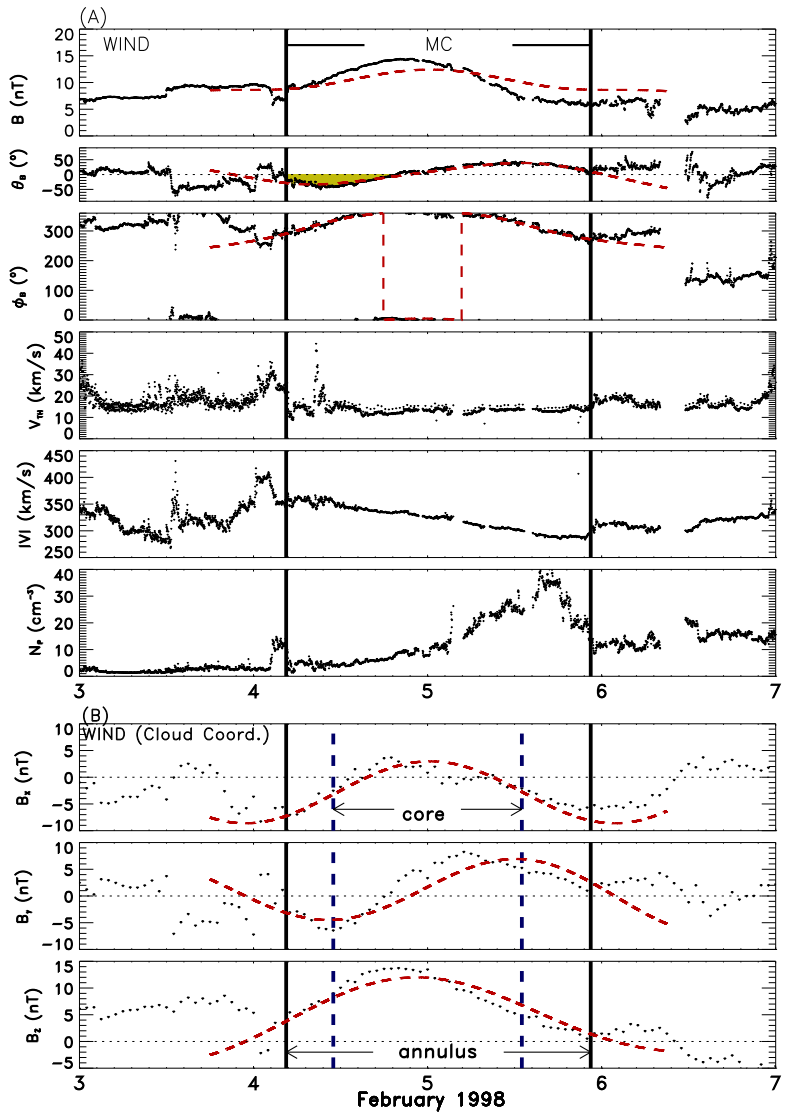

Fig. 8. (A) The magnetic field profile (in GSE coordinates) of a socalled core-annulus MC, in this case occurring on 4 and 5 February Days 35 and 36, 1998, where the (outer) annulus fitting interval is shown by two vertical solid lines, in the same format as Fig. 4 where the red dashed curves are model-fits. The region shaded in yellow is where the observed $\mathrm{B}_{z}$ component is southward. (B) The same MC shown for the magnetic field only and in Cloud coordinates in

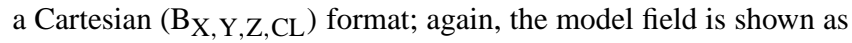
smooth red dashed curves. Now we show two sets of boundaries, the inner (core) boundary and again the outer (annulus) boundary. The max and min in $\mathrm{B}_{\mathrm{Y}, \mathrm{CL}}$ (at the dashed vertical lines, which determine the boundaries of the core) should be in phase with that $\mathrm{B}_{\mathrm{X}, \mathrm{CL}}$-polarity change - as we see they approximately are. There were four examples of this type of MC among the $82 \mathrm{MCs}$; see the asterisks $(*)$ on check $(\mathrm{ck} \%)$ in Table 1.

"Diameter" as a criterion simply addresses size-plausibility (where very large MC diameters, e.g. $\geq 0.45 \mathrm{AU}$, are not believable at $1 \mathrm{AU}$ ) but can be shown to have essentially no correlation with Quality otherwise. The handedness flag (f) and the convergence flag $(\mathrm{F})$ when equal to NOT OK means that all fit-parameters cannot be trusted. It will turn out that 32 out of the full $82 \mathrm{MCs}$ had $\mathrm{Q}_{0}=3$. Of these, 15 violated two or more " $\mathrm{Q}_{0}=3$ " criteria. There were three cases that violated four criteria of Appendix A. For the $32 \mathrm{Q}_{0}=3$ cases, consideration of asf was the most important of the nine criteria listed; 13 violations fell into this category. Consideration of $\chi_{R}$ was second. There were also a lot of MC "checks" violated, but as we shall see, some of them were due to the fact that a MC can have a "core-annulus" structure that we discuss below, and they should not be expected to have a small check value. We now consider an unusual MC structure.

Figure 8A shows the magnetic field and plasma profiles for the MC occurring on 4 and 5 February (Days 35 and 36), 1998. This is a special case of a core-annulus MC, where the solid vertical lines in Fig. 8A denote its outer boundaries. In Fig. 8B we show the MC's magnetic field profile in $\mathrm{Cl}$ coordinates and its model fit (shown by the red dashed curves). Again, two vertical solid lines indicate the outer boundaries, and the traditional fitting interval (enclosing the "core") is given by dashed vertical lines. This structure is compatible with a solution of Eq. (1), but now the outer boundary extends beyond $\mathrm{R}_{0}=2.40 / \alpha$, which is the radius of the core. On the right side of Fig. 6 there is a sketch of the field structure of a core-annulus flux rope approximated by the MC profile of Fig. 8, where the outer radius is taken to be $\mathrm{R}_{\mathrm{NEW}}$. In this case we cannot interpret "check" in the same manner as a MC consisting of only a core. That is, $\mathrm{R}_{\Delta \mathrm{T}}$ in check $\left[=\left(\mathrm{R}_{\Delta \mathrm{T}}-\mathrm{R}_{0}\right) / \mathrm{R}_{0}\right]$ depends on MC duration $(\Delta \mathrm{T})$, which is compatible with the outer boundary of the annulus, but the model- $\mathrm{R}_{0}$ does not depend on $\Delta \mathrm{T}$ and is not directly related to it. However, we have found very few of such core-annulus cases. Possibly only four such cases exist in the WIND data, including one that is apparently an annulus-only-passage (that of 24 June 2000). Table 1 (to be discussed below) denotes which cases fall into the coreannulus (or annulus only) category, by an asterisk (*) marked on the "check" (ck\%) value for that MC.

We point out a characteristic property of the Lepping et al. (1990) fitting program that is most helpful at times, i.e. its ability to attempt to provide the correct output MC parameter estimates, even though the wrong analysis interval (i.e. wrong start- or end-point) is chosen for the fitting. This is more likely to happen when one of the boundaries is chosen correctly. For example, see Fig. 9 which shows such a case, the MC of 27 May 1996. At the top of Fig. 9A the full (apparently correct) interval of the MC was employed, where the duration was a long $40 \mathrm{~h}$ (at a slow average speed of $370 \mathrm{~km} / \mathrm{s}$ ), and this lead to a successful fit. At the bottom of Fig. 9 (B) only part of the MC was used for the fitting with the same model-program, where the duration was incorrectly assumed to be $21 \mathrm{~h}$. It is interesting that trial B provides fit-parameters that are very similar to those of trial $\mathrm{A}$. That is, for interval $\mathrm{A}, \mathrm{R}_{0}=0.17 \mathrm{AU},\left|\mathrm{Y}_{0} / \mathrm{R}_{0}\right|=10.5 \%, \mathrm{~B}_{0}=11.9 \mathrm{nT}$, $\left(\phi_{A}, \theta_{A}\right)=\left(120^{\circ}, 32^{\circ}\right), \quad \beta_{C A}=115^{\circ}$, and $\left|\chi_{R}\right|=0.181$, and for interval $\mathrm{B}$, these were $\mathrm{R}_{0}=0.15 \mathrm{AU},\left|\mathrm{Y}_{0} / \mathrm{R}_{0}\right|=4.5 \%$, $\mathrm{B}_{0}=12.2 \mathrm{nT}, \phi_{A}, \theta_{A}=\left(108^{\circ}, 40^{\circ}\right), \beta_{C A}=103^{\circ}$, and $\left|\chi_{R}\right|=0.121$, showing relatively small differences from trial $A$, even though the input "analysis interval" was almost halved from trial A to B. And it is obvious that both trials give very similar field profiles for the shorter interval, and they continue to agree upon extrapolation of trial-B's model field (not shown) over the full length of trials interval. However, as should be expected, "check" was vastly different between the two trials: it was -0.07 (acceptable) for vs. -0.40 (very poor) for B. 
- $\quad$ NmNNMN-MNN

"

"

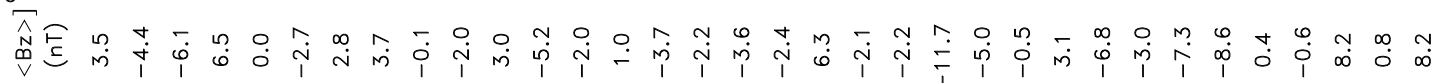

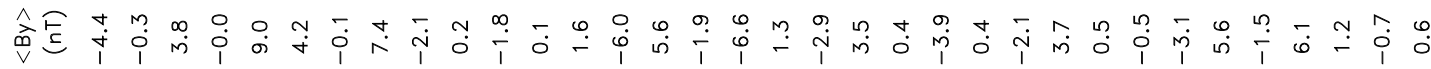

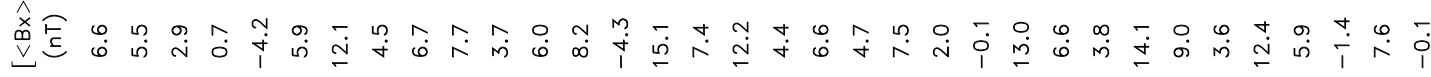

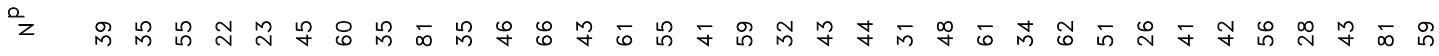

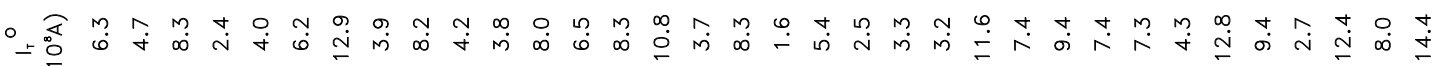

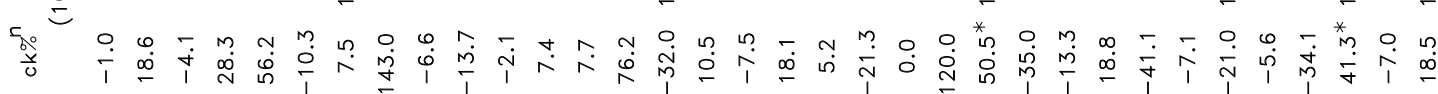

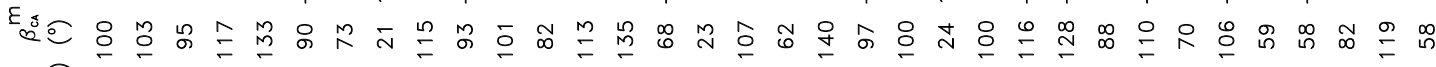

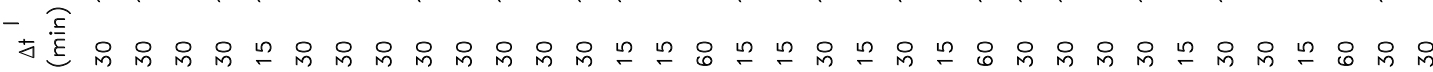

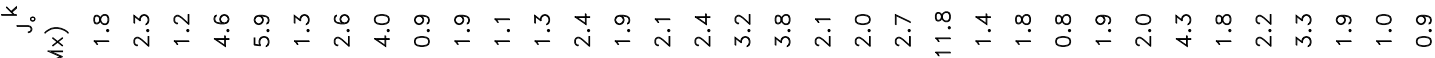

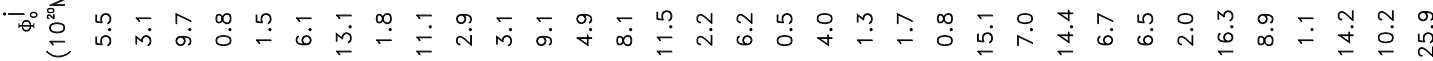

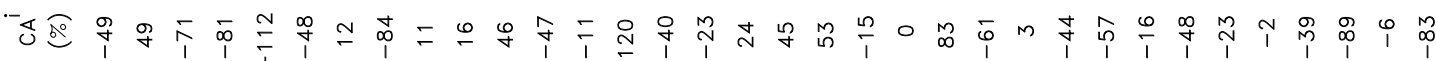

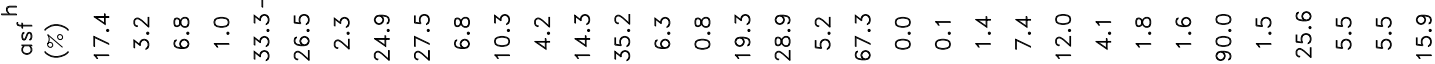

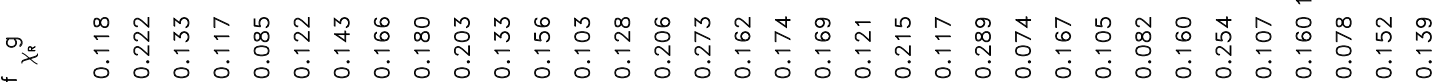

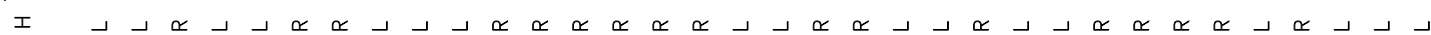

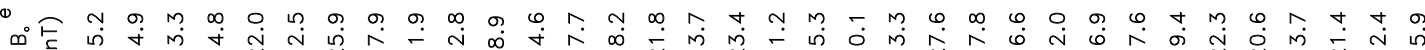
。

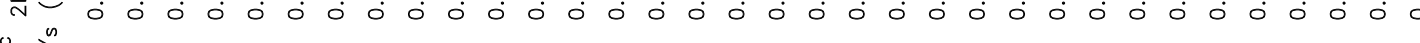

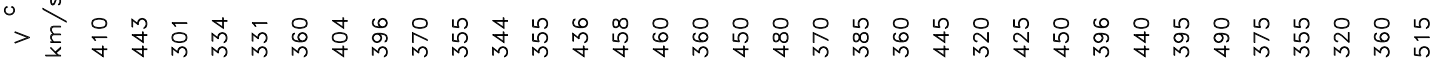

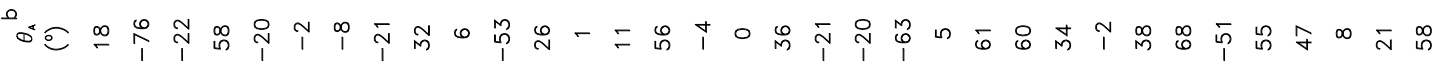

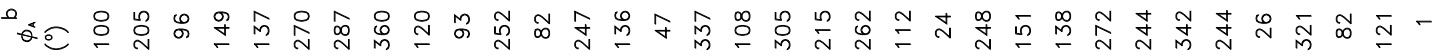

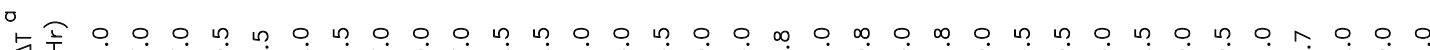

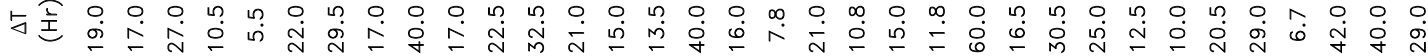

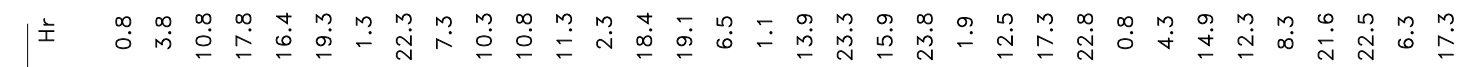

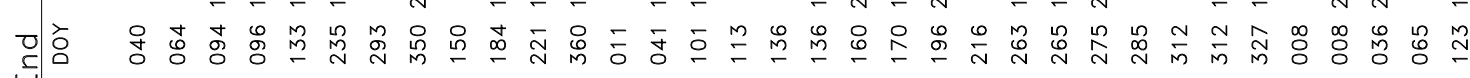
山ै

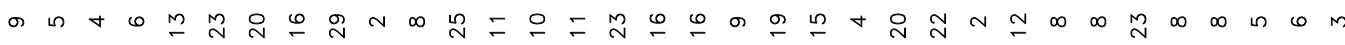

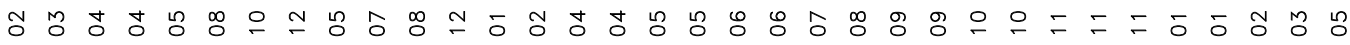

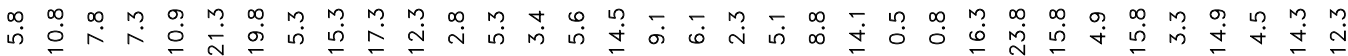

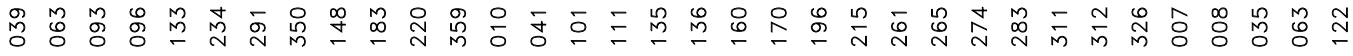

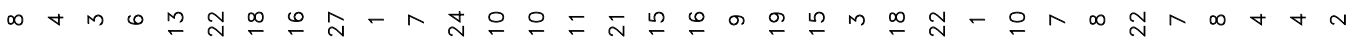

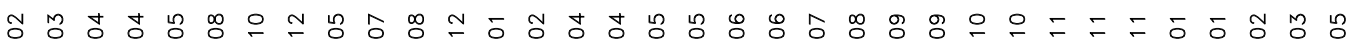

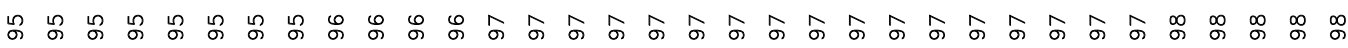


$+\infty$

$\omega_{L}$ 。 "

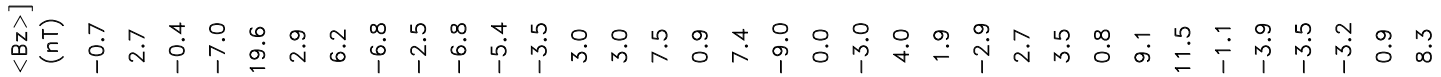

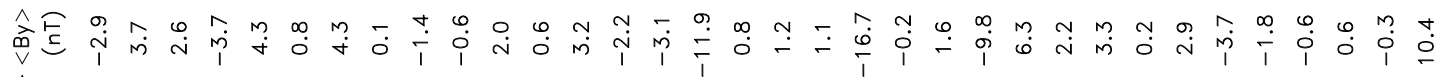

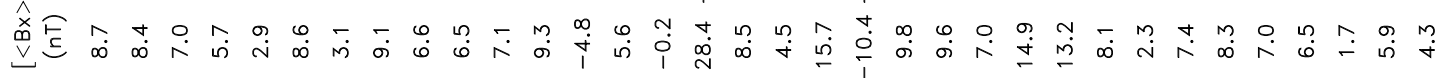

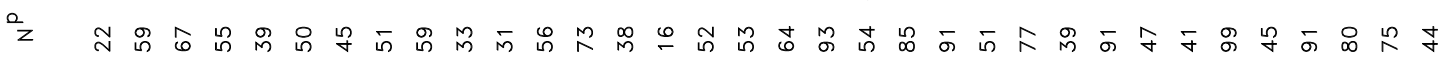

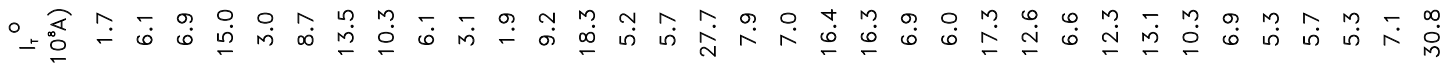

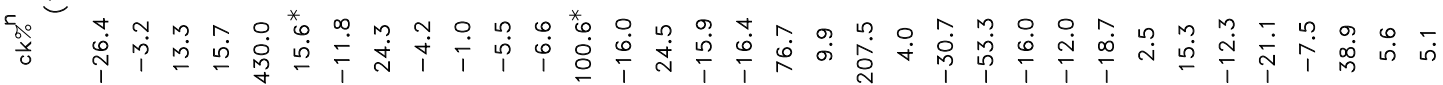

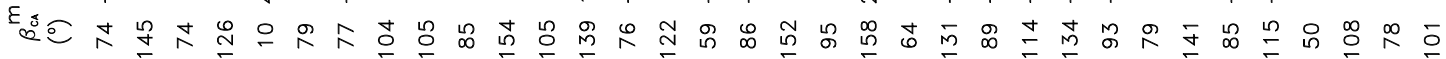
- $\frac{\widehat{c}}{\bar{\varepsilon}}$

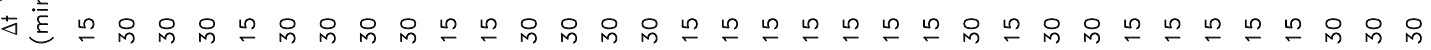

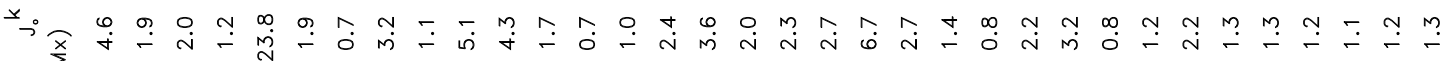

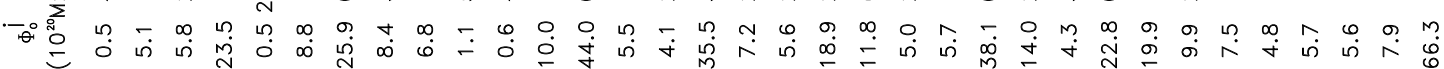

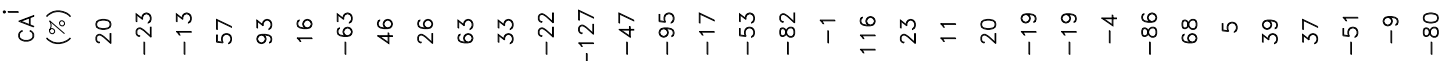

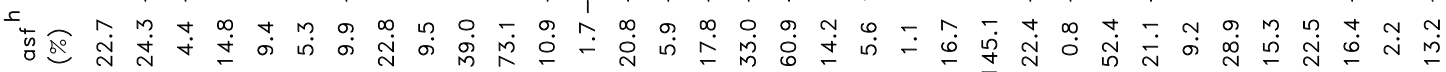
$\sigma_{x} \quad$ o ※ I $\lrcorner\lrcorner \propto\lrcorner \propto \propto\lrcorner\lrcorner\lrcorner\lrcorner\lrcorner \propto\lrcorner\lrcorner\lrcorner\lrcorner\lrcorner \propto\lrcorner\lrcorner \propto \propto\lrcorner\lrcorner\lrcorner\lrcorner\lrcorner \propto\lrcorner\lrcorner\lrcorner \propto\lrcorner\lrcorner$ o மீ结

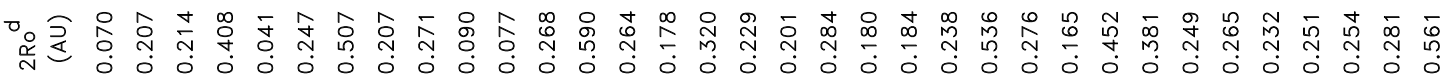

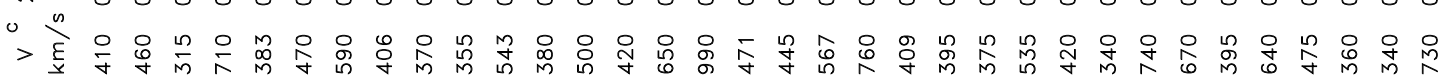

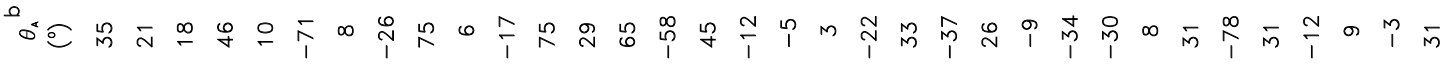

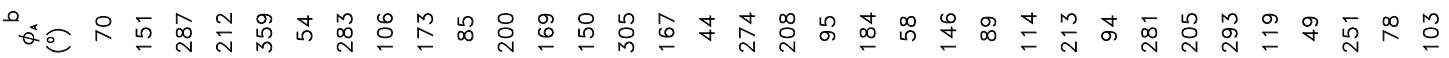

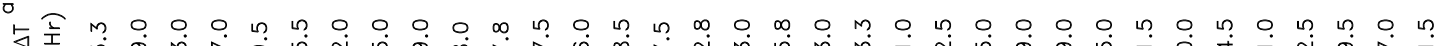
$\checkmark$ ம

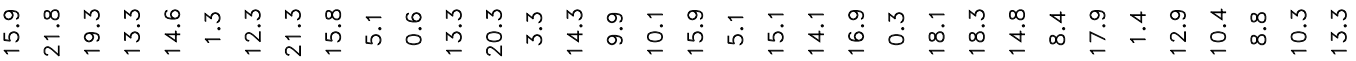

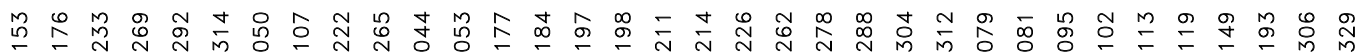
N N 용

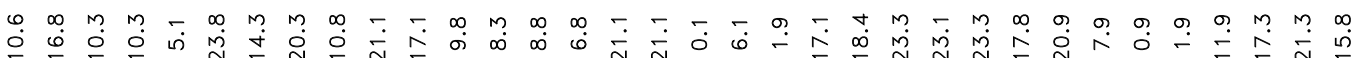
员 ⿱ㅗㄷ N

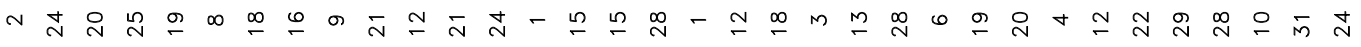

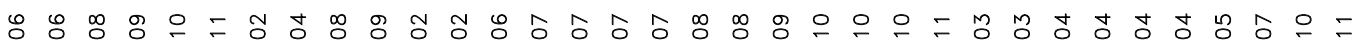

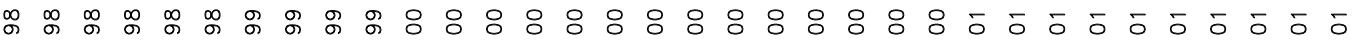


$+0 \quad n N-m-m m n N m-m m N$

$n$ ¿

"

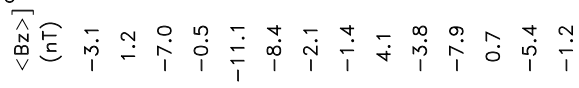

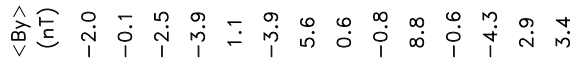

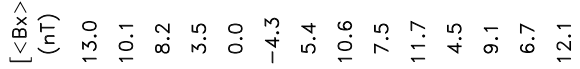

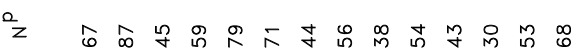

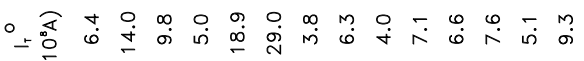

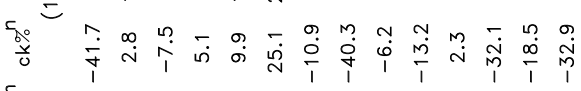

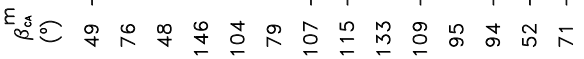

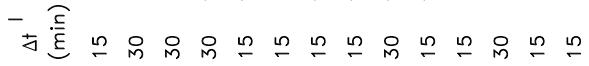

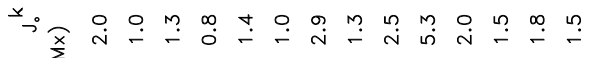

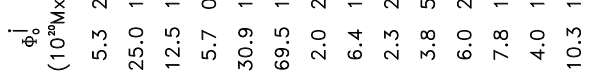

-ব大

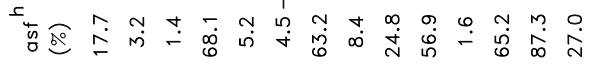

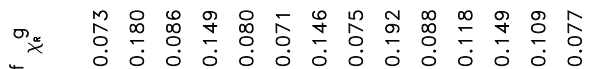

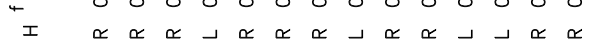

œ

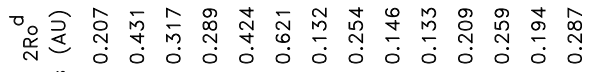

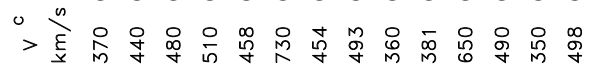

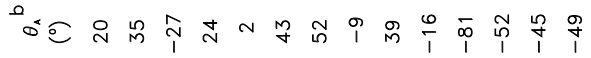

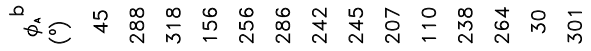

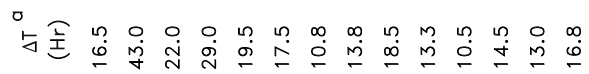

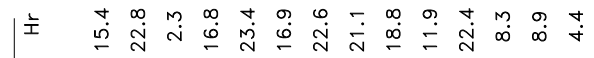

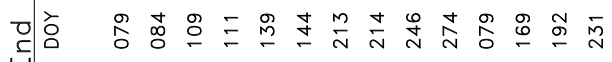

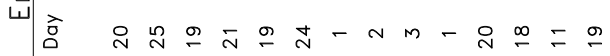

ㅇำ

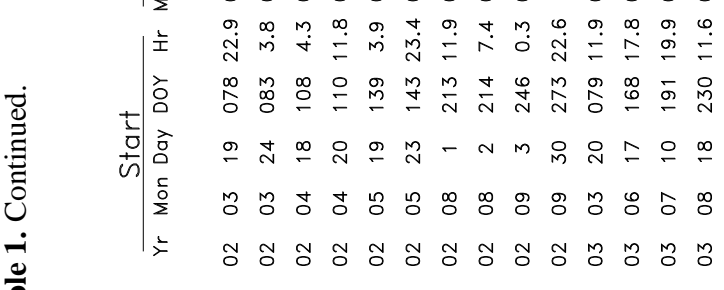

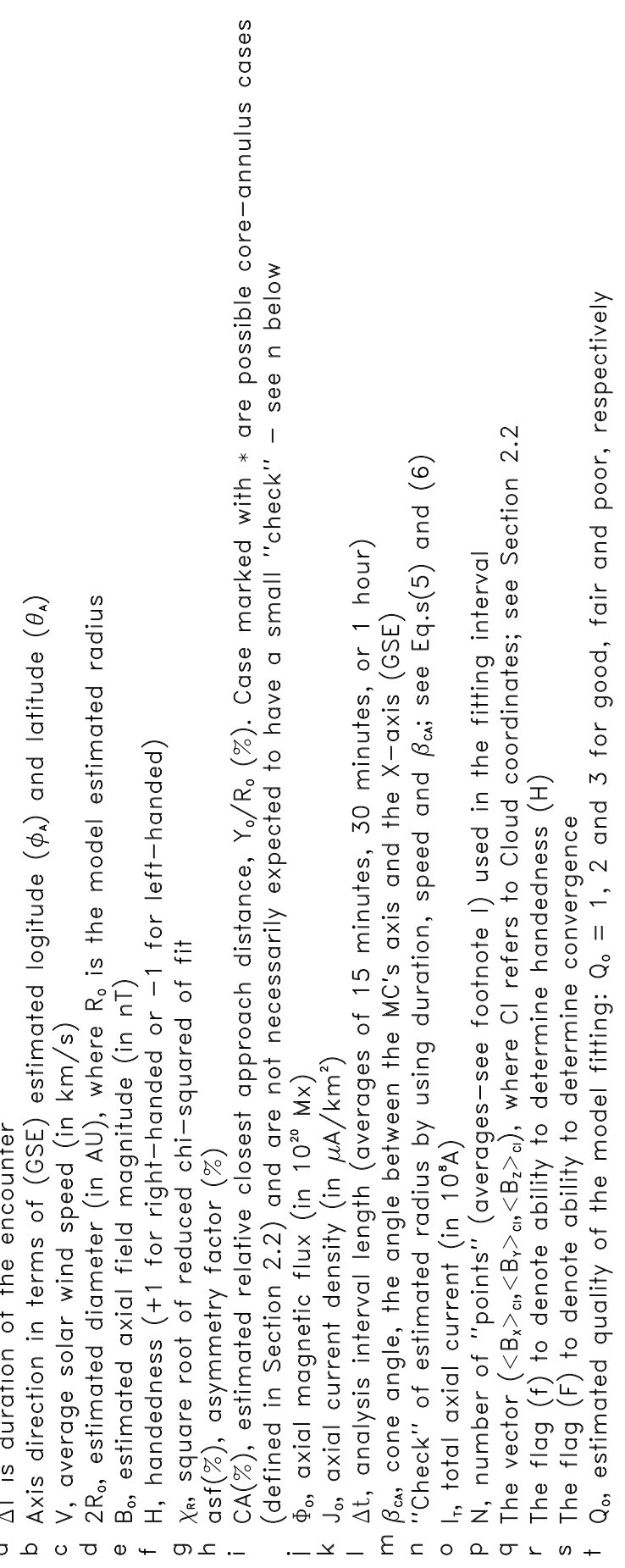


This difference in "check" is a warning that the B-interval was probably incorrect, and similarly for asf (the asymmetry factor), where asf was $27.5 \%$ for interval $\mathrm{A}$, but had a ridiculous value of $122 \%$ for interval $\mathrm{B}$, again as might be expected.

\section{Average characteristics of magnetic clouds}

The MCs studied here are from WIND data from early 1995 to August 2003, i.e. covering parts of both the quiet and active parts of the solar cycle. They were initially identified by visual examination of magnetic field and plasma data by at least two members of the WIND/MFI team based on the Burlaga (1988) definition of a MC.

\subsection{Summary of model-related magnetic cloud characteristics}

Lepping and Berdichevsky (2000) presents a preliminary statistical examination of WIND MC parameters (for only $28 \mathrm{MCs}$ ), along with those for MCs from an earlier period mostly observed by the IMP- 8 spacecraft. Table 1 is a summary listing of the full 82 WIND MCs with specific event times, durations, model fit-parameters and various related quantities; in footnotes at the bottom of the table is the particular quantity's definition. All of the quantities needed to obtain $\mathrm{Q}_{0}$ (in last col., footnote $\mathrm{r}$ ) are contained in Table 1. Table 2 gives the averages and standard deviations for most of these quantities for the full $82 \mathrm{MCs}$ for both the full set of $82 \mathrm{MCs}$ and for the $\mathrm{Q}_{0}=1,2$ set (i.e. excluding poor cases) separately. These averages may be compared to those given in the Lepping and Berdichevsky (2000) paper, which showed preliminary values for WIND MC fitparameters for the early part of the mission (1995-1998) vs. those from Lepping et al. (1990) mainly from IMP 8 (see Table 1 in that paper) for the earlier interval 1967-1982, primarily in a period of greater solar activity. Lepping and Berdichevsky (2000) show that, from the active (IMP) to the mostly quiet (WIND) period, $\mathrm{B}_{0}$ decreases by about $16 \%$ and MC speed decreases by $11 \%$. Our present Table 2 (where there is overlap in quantities) supports most of the values in Table 2 of Lepping and Berdichevsky (2000), but apparently is a little closer to the IMP set, except for the average of $\Phi_{0}$. We note that on average these MCs are one day long, 1/4 AU in diameter, show a slight preference for having axes close to Y-axis(GSE) alignment, and carry an axial current $\left(\mathrm{I}_{T}\right)$ of about a billion amps, where $\mathrm{I}_{T}=0.432 \pi \mathrm{J}_{0} R_{0}^{2}$ derived from the Lepping et al. (1990) model. The MCs have a broad range of speeds, but speed was typically around $400 \mathrm{~km} / \mathrm{s}$ on average early in WINDs mission (Lepping and Berdichevsky, 2000). It has climbed to significantly larger values recently, giving an 8.6-year-average of $453 \mathrm{~km} / \mathrm{s}$.

In Fig. 10 we display the distributions of the estimated fit-parameters and other relevant quantities comprising the following: Duration $(\Delta \mathrm{T})$, axis direction in terms of longitude and latitude $\left(\phi_{A}, \theta_{A}\right.$ in GSE coordinates), average
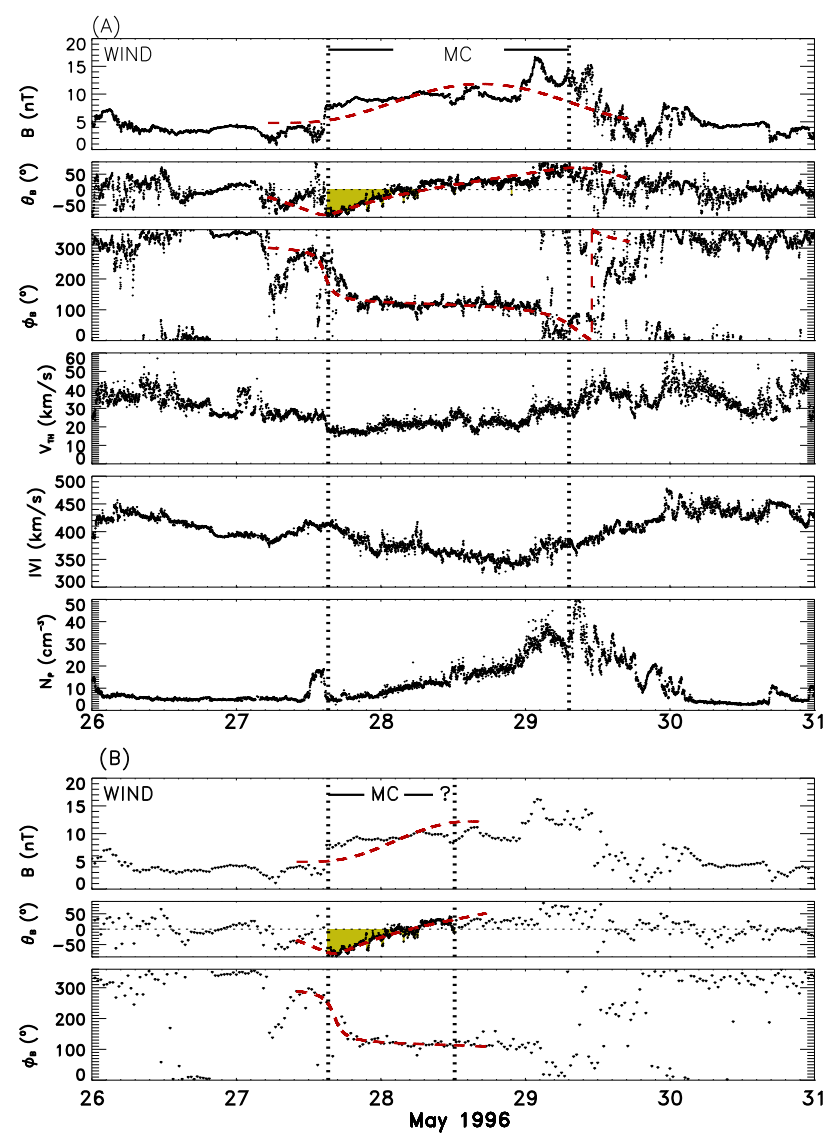

Fig. 9. Two attempts to fit the same MC: (A) Comparison between the observed field and the force-free MC model fit (smooth dashed curves) to these data in terms or field magnitude $(|\mathbf{B}|)$ and direction (longitude and latitude angles, $\phi_{\mathrm{B}}, \theta_{\mathrm{B}}$ ) (also shown: proton thermal speed, bulk speed, and density) for the MC of 27 May 1996. The fitted curves hold strictly only within the vertical dashed lines, which represent the estimated endpoints of the MC. The full interval of this $\mathrm{MC}$, where the duration was a long $40 \mathrm{~h}$, is fitted with moderately good success. (B) Now for convenience only $30 \mathrm{~min}$.- averages of the field are shown. In (B) only part of the MC was fitted with the same model, where the duration was incorrectly assumed to be $21 \mathrm{~h}$, which interestingly provided almost the same fit-parameters values as those from the (A)-interval fitting, including very nearly the same estimated radius. The model occasionally displays this robust feature. This is a $\mathrm{Q}_{0}=2 \mathrm{MC}$. The regions shaded in yellow are where the observed $\mathrm{B}_{z}$ component is southward.

MC speed $(\mathrm{V})$, diameter $\left(2 \mathrm{R}_{0}\right)$, axial field magnitude $\left(\mathrm{B}_{0}\right)$, square-root of chi-squared of the fit $\left(\chi_{R}\right)$, asymmetry factor $(\operatorname{asf}(\%))$, relative closest approach distance CA $(\%)$, axial magnetic flux $\left(\Phi_{0}\right)$, current density on the axis $\left(\mathrm{J}_{0}\right)$, axis cone angle $\left(\beta_{C A}\right)$ with respect to the $\mathrm{X}(\mathrm{GSE})$-axis, radius"check $(\%)$ ", average of the MC's field in Cloud coordinates, $\left(<\mathrm{B}_{X}>_{C l},<\mathrm{B}_{Y}>_{C l},<\mathrm{B}_{Z}>_{C l}\right)$; see Sect. 2.2 for a definition of magnetic cloud coordinates. We now discuss some features of the distributions in Fig. 10.

The $\Delta \mathrm{T}$-distribution is that of a severely skewed gaussian $(\approx$ Poisson) with a mode around $15 \mathrm{~h}$. Only one case went above $50 \mathrm{~h}$. The long durations are generally indicative of the 
Table 2. Averages and $\sigma$ 's of MC fit-parameters of Table 1.

\begin{tabular}{|c|c|c|c|c|}
\hline \multirow[b]{2}{*}{ Quantity } & \multicolumn{2}{|c|}{ Full Set $(N=82)$} & \multicolumn{2}{|c|}{$Q_{0}=1,2$ Set $(N=50)$} \\
\hline & Average & $\sigma$ & Average & $\sigma$ \\
\hline$\Delta \mathrm{T}$ (Hours) & 21.1 & 10.7 & 21.6 & 9.3 \\
\hline$\phi_{A}\left({ }^{\circ}\right)<180^{\circ}$ & 99 & 7 & 100 & 8 \\
\hline$\phi_{A}\left({ }^{\circ}\right) \geqq 180^{\circ}$ & 265 & 7 & 263 & 8 \\
\hline$\theta_{A}\left({ }^{\circ}\right)$ & 6 & 38 & 8 & 39 \\
\hline$\left|\theta_{A}\right|\left(^{\circ}\right)$ & 31 & 22 & 32 & 23 \\
\hline$\beta_{\mathrm{cA}}\left({ }^{\circ}\right)$ & 95 & 32 & 96 & 26 \\
\hline$V(\mathrm{~km} / \mathrm{s})$ & 453 & 124 & 455 & 129 \\
\hline $2 R o(A U)$ & 0.249 & 0.122 & 0.249 & 0.078 \\
\hline$B_{0}(n T)$ & 17.8 & 6.9 & 17.1 & 5.9 \\
\hline$\chi_{R}$ & 0.138 & 0.053 & 0.124 & 0.040 \\
\hline $\operatorname{asf}(\%)$ & 22.2 & 29.6 & 12.1 & 9.1 \\
\hline$|C A(\%)|$ & 43.4 & 33.3 & 36.0 & 26.3 \\
\hline$I_{T}\left(10^{8} \mathrm{~A}\right)$ & 8.57 & 5.72 & 8.37 & 4.47 \\
\hline$\Phi_{0}\left(10^{20} M x\right)$ & 10.7 & 12.8 & 9.2 & 7.2 \\
\hline$J_{0}\left(\mu A m p / \mathrm{km}^{2}\right)$ & 2.53 & 2.95 & 1.95 & 0.91 \\
\hline |check(\%)| & 29.4 & 57.1 & 15.4 & 12.1 \\
\hline $\mid<B x>I_{c L}(n T)$ & 7.20 & 4.49 & 8.25 & 4.90 \\
\hline $\mid<B y>I_{C L}(n T)$ & 2.98 & 3.10 & 2.24 & 2.15 \\
\hline $\mid<B z>I_{c L}(n T)$ & 4.19 & 3.40 & 3.88 & 2.87 \\
\hline $\mathrm{N}$ & 53.0 & 18.9 & 55.7 & 20.0 \\
\hline
\end{tabular}

large cross-sections of some of these structures, but some are a reflection of the slow speeds of some MCs, as clearly seen in Table 1. The $\phi_{A}$-distribution shows an obvious bimodal structure due to the fact that when the MC is seen at or near its "nose", globally speaking, and especially when having inclinations not far from the ecliptic plane, the axis will likely be near $\phi_{A}=90^{\circ}$ or $270^{\circ}$, as in fact we see in the histogram. But the histogram shows many cases between these modes as well. It is interesting that the $\theta_{A}$-distribution is asymmetric, strongly favoring positive values which is likely due to the phase of the Suns dipolar field; however, this suggestion must be subjected to critical analysis. The V-distribution shows a broad peak around $400 \mathrm{~km} / \mathrm{s}$, ending at about $500 \mathrm{~km} / \mathrm{s}$. As we moved into the active part of the cycle we occasionally saw faster speeds, as Table 1 shows (in the $\mathrm{V}$ column), providing the $\mathrm{V}$-distributions long tail. The $2 \mathrm{R}_{0}$-distribution, with a peak at about $0.20 \mathrm{AU}$, is similar in structure to the $\mathrm{V}$ distribution, but there is an abrupt drop around $2 \mathrm{R}_{0}=0.30 \mathrm{AU}$; this is not surprising since an expanding MC (which most of them are at $1 \mathrm{AU}$ ) would not have had enough time to expand to diameters greater than about $0.3 \mathrm{AU}$ in going from the Sun to $1 \mathrm{AU}$. At farther distances from the Sun we would expect there to be a higher percentage of larger cross-sections. The $\mathrm{B}_{0}$-distribution is rather smoothly changing from the most probable value at about $13 \mathrm{nT}$ to very high, but uncommon, values, such as were seen in the second Bastille Day MC when $\mathrm{B}_{0}$ reached a value near $50 \mathrm{nT}$ (e.g. Lepping et al., 2001; Mulligan et al., 2001). The $\chi_{R}$-distribution displays a rather restricted range of values between 0.05 and 0.2 with only a few beyond that value, to 0.3 . This is a measure of the rather narrow range of field directional variations (which $\chi_{R}$
Table 3. Magnetic Cloud $\Theta_{B}$ (or $B_{\mathrm{Z}}$ ) Categories*.

\begin{tabular}{clcl} 
Category no. & Definition & Category no. & Definition \\
1 & $\mathrm{~N} \Rightarrow \mathrm{S}$ & 11 & $\mathrm{~S} \Rightarrow \mathrm{N}$ \\
2 & $\mathrm{~N} \Rightarrow \mathrm{S}$, mostly $\mathrm{N}$ & 12 & $\mathrm{~S} \Rightarrow \mathrm{N}$, mostly $\mathrm{S}$ \\
3 & Almost all N & 13 & Almost all S \\
4 & All N & 14 & All S \\
5 & $\mathrm{~N} \Rightarrow \mathrm{S}$, mostly $\mathrm{S}$ & 15 & $\mathrm{~S} \Rightarrow \mathrm{N}$, mostly N \\
& \multicolumn{3}{l}{} \\
* The Categories were qualitatively determined by visual inspection.
\end{tabular}

measures - not variations in B-intensity, as we pointed out above) in our set. The asf(\%)-distribution is of a simple form, such as $1 /(\mathrm{K}+\mathrm{asf})^{k}$, where $\mathrm{K}$ and $\mathrm{k}$ are constants, and $\mathrm{k}$ is apparently close to unity. So a large percent of cases have relatively good symmetry. The CA(\%)-distribution is approximately normal (at least more so than any other distribution in Fig. 10, excluding those of the averages of $\mathbf{B}$ in the MCs in $\mathrm{Cl}$ coordinates). One might have expected a more uniform distribution, especially if there is no bias in solar source expulsion direction. We point out that $\mathrm{CA}(\%)$ is not an intrinsic characteristic of MCs, so its observed (but unexpected) distribution probably reflects an approximate realization of at least the following two factors: 1) any natural focusing of the path of the related ejecta toward (or possibly away from) the ecliptic plane and more likely 2) any observer-selection effect, whereby the MCs with small CA(\%) are easier to recognize than those at or near $100 \%$. The $\beta_{C A}$-distribution is a slightly skewed normal distribution where the peak is very near $90^{\circ}$ which should be expected for the type of $\phi_{A}$, $\theta_{A}$-distributions seen here and according to $\beta_{\mathrm{CA}}$ 's definition (see Eq. 3); $\beta_{C A}$ should be somewhat broadly distributed and peak near $90^{\circ}$, as it is seen to do. The distributions of $\Phi_{O}$ and $\mathrm{J}_{0}$ are of similar structure and drop off rapidly, except for a low number of cases in their long tails, i.e. there are only a small number of high values. The "check(\%)"-distribution is relatively narrow and has a peak near zero, as expected. But interestingly, when $\mid$ check $\mid<100 \%$ (i.e. reasonable values), check very much favors negative values, which means that most of the time for reasonable cases $\mathrm{R}_{\Delta \mathrm{T}} \leq \mathrm{R}_{0}$ (see Eq. 6). When $\mid$ check $\mid>100 \%$, it is observed that $\mathrm{R}_{\Delta T}>\mathrm{R}_{0}$, and then most of such cases are either core-annulus types [discussed above in Sect. 3.0 (and see Figs. 6 and 8), and for which we cannot depend on check for judging quality], or they are simply poor cases. The distributions of $\left\langle\mathrm{B}_{x}\right\rangle_{C l},\left\langle\mathrm{~B}_{y}\right\rangle_{C l}$, and $<\mathrm{B}_{z}>_{\mathrm{Cl}}$ in Cloud coordinates (see the last three panels) are all approximately normally distributed and appear to have reasonable peak values. Most of the cases where $<\mathrm{B}_{x}>_{\mathrm{Cl}}<0$ are poor cases, except where a core-annulus case is considered.

Figure 11 shows most of the quantities in Fig. 10 as a function of year for the period from 1995 to August of 2003; the last three panels of the two figures are different, which in Fig. 11 are: (panel n) handedness, $H$, (o) total axial current, $\mathrm{I}_{\mathrm{T}}$, and (p) quality, $\mathrm{Q}_{0}$. Many of the panels of Fig. 11 appear to show a rough tendency of many of the parameters to split into three regions: early, middle, and late, where the middle 

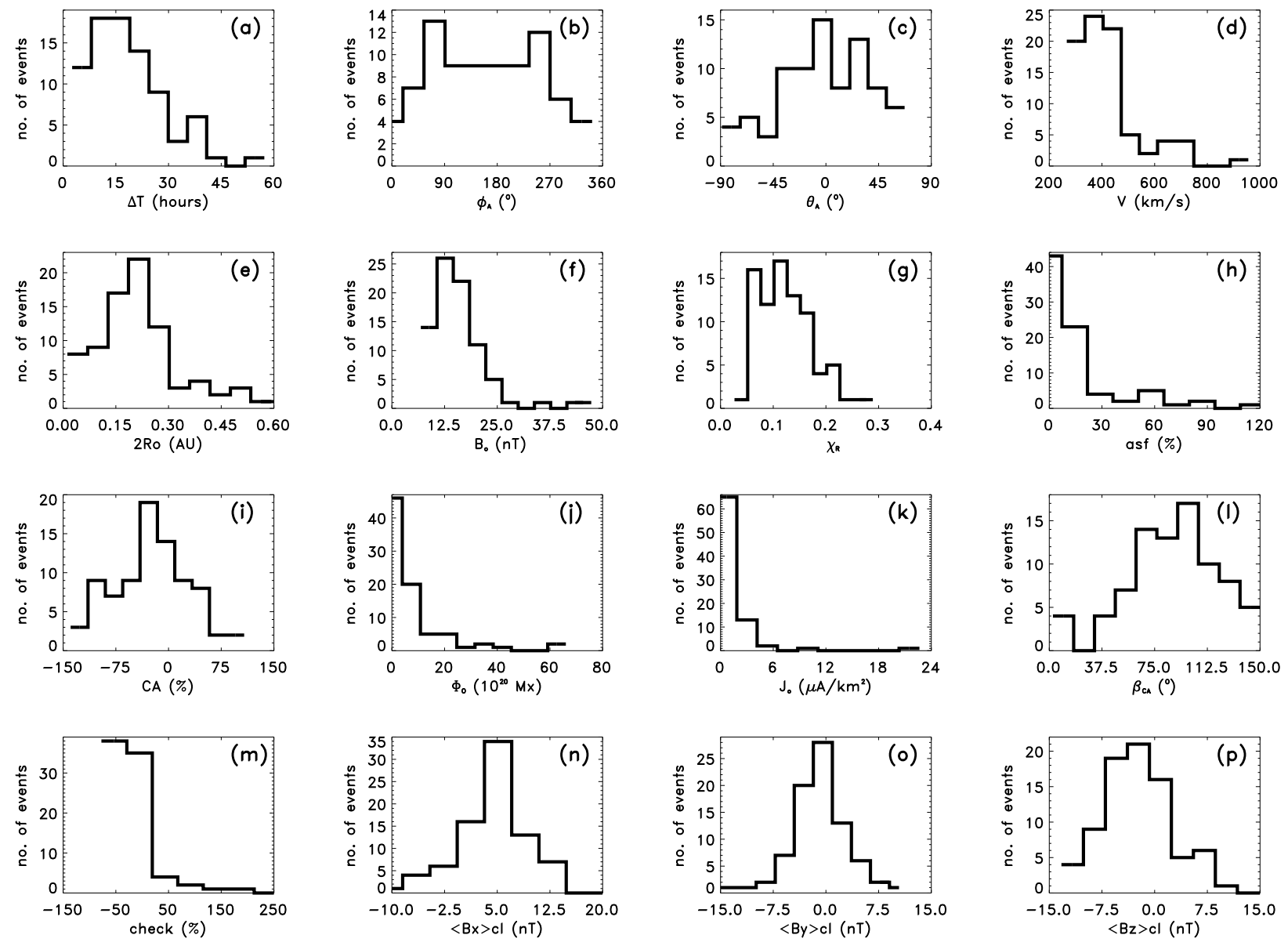

Fig. 10. Histograms of various estimated fit-parameters, by the Lepping et al. (1990) MC model, and related quantities for the 82 MCs for the period from 1995 to August 2003 given in panels (a) through (p), respectively as: Duration $(\Delta \mathrm{T})$, axis direction in terms of (GSE) longitude and latitude $\left(\phi_{\mathrm{A}}, \theta_{\mathrm{A}}\right)$, average speed $(\mathrm{V})$, diameter $\left(2 \mathrm{R}_{0}\right)$, axial field magnitude $\left(\mathrm{B}_{0}\right)$, square root of reduced chi-squared of fit $\left(\chi_{R}\right)$, asymmetry factor $(\operatorname{asf}(\%))$, relative closest approach distance $\mathrm{CA}(\%)$, axial magnetic flux $\left(\Phi_{0}\right)$, current density on the axis $\left(\mathrm{J}_{0}\right)$, axis cone angle $\left(\beta_{C A}\right)$ with respect to the $\mathrm{X}(\mathrm{GSE})$-axis, radius-check $(\%)$, average of the MC's field in Cloud coordinates, $\left(<\mathrm{B}_{X}>_{C l},<\mathrm{B}_{Y}>_{C l}\right.$, $<\mathrm{B}_{\mathrm{Z}}>_{\mathrm{Cl}}$ ); see the text for a definition of Cloud coordinates.

region (most apparent in $\phi_{\mathrm{A}}$, panel (b), where $<\phi_{\mathrm{A}}>$ tends toward $180^{\circ}$, whereas it was usually bimodal with peaks near $90^{\circ}$ and $270^{\circ}$. This middle region occurs over the interval from late 1998 to early 2000. But part (or all?) of this difference in appearance, however, is apparently due to a paucity of MCs in that time period. This apparent split is not easily quantified. In panel $(\mathrm{n})$ handedness $(\mathrm{H}$, or chirality, where +1 is $\mathrm{R}$ and -1 is $\mathrm{L}$ ) shows an apparent oscillation between one type and the other for most of the mission but is of only $\mathrm{H}=\mathrm{L}(-1)$ for the middle region. This temporal distribution is not completely understood, but clearly it depends on the vector nature of the solar source regions (see Bothmer and Rust, 1997) and on the specific origin of the MCs, where the $H=R$ cases are expected to arise in the Southern Hemisphere of the Sun and the $\mathrm{H}=\mathrm{L}$ cases from the Northern Hemisphere. The quantity that shows the clearest pattern is speed, V, in panel (d). The envelope of V markedly increases from 1995 to the end of the data, almost linearly, except for the Bastille Day event where V was near $1000 \mathrm{~km} / \mathrm{s}$.

\subsection{Classified by Category and "State"}

We find it useful to categorize MCs according to a combination of handedness $(\mathrm{H})$ and $\theta_{\mathrm{B}}$ profiles; see Fig. 4 of Lepping et al., 2005 that preliminarily examines MC profiles in these terms. The $\theta_{\mathrm{B}}$ profiles can be put into 10 different Categories, as shown in Table 3, and likewise $\mathrm{H}$ put into two. The distributions of these Categories for our $82 \mathrm{MCs}$ is shown in Table 2 of Lepping et al. (2005); also see Figs. 10 and 11 of Lepping et al. (2003b), which show pictorially how the associated MC flux ropes are oriented with respect to the Ecliptic plane to create such Categories. Therefore we have a total of $10 \times 2=20$ possible Categories. We then define a State function as

State $=$ Category $\times H$,

where $\mathrm{H}$ is +1 (Right-handed) or -1 (Left-handed) and "Category" is assigned, for example, 1 for $\mathrm{N} \rightarrow \mathrm{S}, 11$ for $\mathrm{S} \rightarrow \mathrm{N}$, 3 for almost all N, etc.; see the top part of Fig. 12 which 

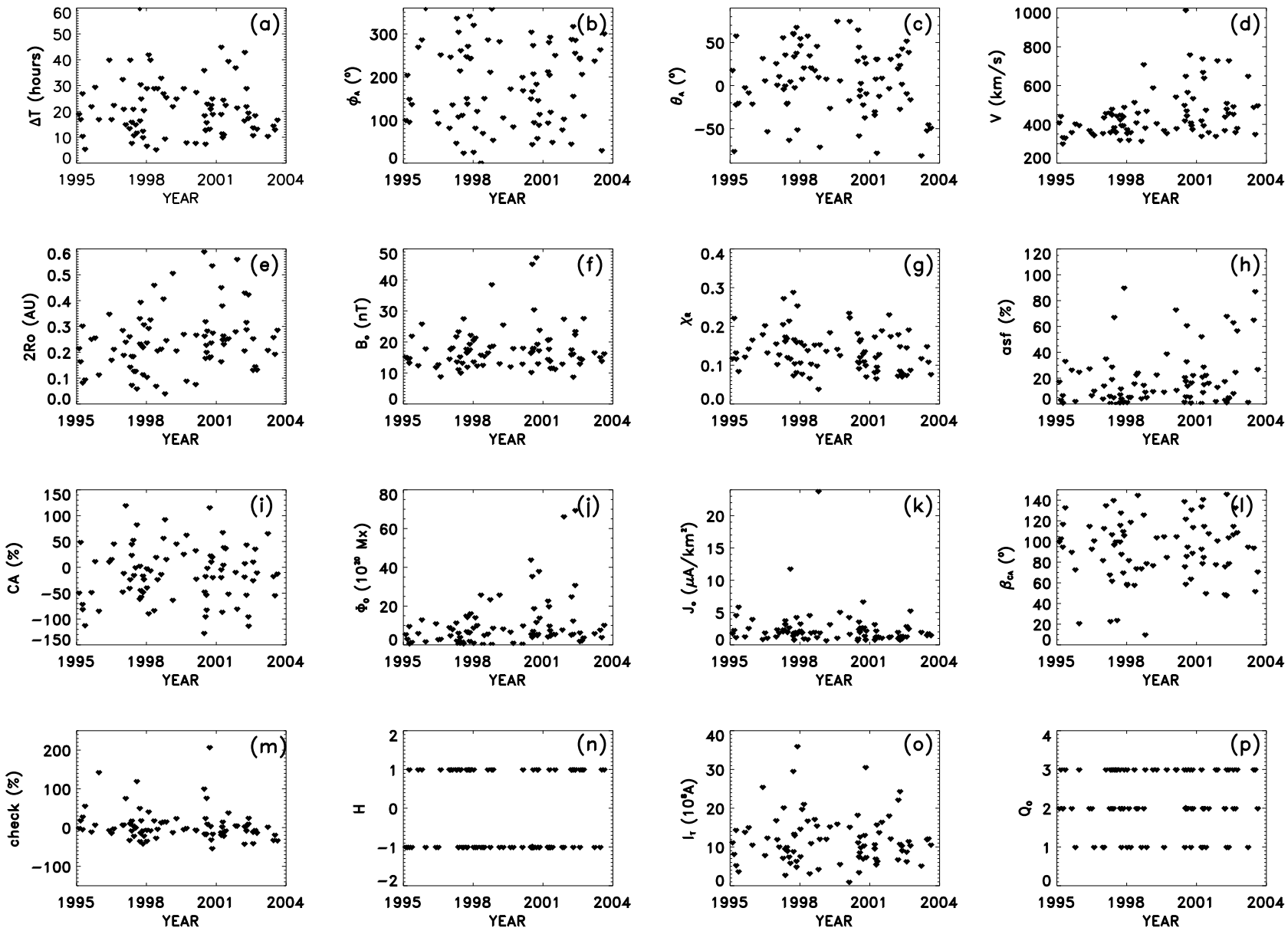

Fig. 11. Variation of various MC quantities vs. time for all 82 cases; the quantities match those of Fig. 10, except for the last three, where the histograms in Fig. 10 of $\left\langle\mathrm{B}_{X}>_{C l},<\mathrm{B}_{Y}>_{C l}\right.$, and $\left\langle\mathrm{B}_{Z}>_{C l}\right.$, respectively, are now replaced by: (n) handedness [H]; (o) total axial current $\left[\mathrm{I}_{T}\right]$; and (p) quality $\left[\mathrm{Q}_{0}\right]$.

defines 10 different Categories, 1, 2, 3, 4, 5 and 11, 12, 13, 14,15 . Hence, State ranges over -15 to $-11,-5$ to +5 and +11 to +15 , in increments of 1 . In some notation schemes an $\mathrm{S} \rightarrow \mathrm{N}$ Category with $\mathrm{H}=+1$ (right-handed) would be called an SWN MC and a SEN MC is in a $\mathrm{S} \rightarrow \mathrm{N}$ Category with $\mathrm{H}=-1$ (left-handed), similarly for the two $\mathrm{N} \rightarrow \mathrm{S}$ types; see, e.g. Mulligan et al. (1998) and Bothmer and Rust (1997). Our notation allows us to examine a larger set of Categories, covering MCs that are steeply inclined. See the bottom of Fig. 12 which gives State as a function of time and showing some obvious clustering of this quantity in at least five places; clustering here means a tightly grouped set of 3 or more MCs in a given State in a 14 month (or shorter) period, but not exclusively of that State. Most of these (4 sets) are in the \pm 11 State, i.e. from Category 11 ( $\mathrm{S} \rightarrow \mathrm{N}$ types), where two had $\mathrm{H}=-1$ and two had $\mathrm{H}=+1$, but two sets of MCs, in 1998 and around late 2000, have States of +4 (all $\mathrm{N}$ ) with $\mathrm{H}=+1$. So sometimes there was a slight tendency for the MCs to fall into sets of a particular Category with a fixed handedness, but with some small amount of intermixing of other Categories as well. Clustering of Categories 4 and 11 should not be surprising, since they have been the two most commonly occurring types of MCs, as the top of Fig. 12 shows.

Since we have the ability to transform from GSE to Cloud coordinates (as described in Sect. 2.3) and since our MC parameter fit program gives $\left|\mathrm{Y}_{0}\right|$ and the sign of $\mathrm{Y}_{0}$ in Cloud coordinates, we are able to find the closest approach vector in GSE coordinates by an inverse transformation of the vector $\boldsymbol{Y}_{0}$ (Cloud coordinates). Table 4 gives details on $\mathrm{MC} \mathrm{Q}_{0}$ and State (see Eq. 9), as well as this vector information on the closest approach of the spacecraft to the MCs axis for all 82 cases. The table gives the closest approach unit vector in GSE coordinates (yy,zz) and the actual closest approach vector (again in GSE) in $A U$ units $(Y, Z)$, and provides the scalar closest approach, $C A=\mathrm{Y}_{0} / \mathrm{R}_{0}(\%)$ (also provided in Table 1 ) for completeness; the sign on $\mathrm{Y}_{0} / \mathrm{R}_{0}(\%)$ refers to closest approach on the positive (i.e. $\left.+\mathbf{Y}_{C l}\right)$ or negative $\left(-\mathbf{Y}_{C l}\right)$ side of the MCs axis. Notice that since the MCs are assumed to be moving locally along the $\mathrm{X}$-axis(GSE), there are no $x$-components in GSE coordinates for the closest approach vectors. This ability to provide $\mathrm{CA}$ in various forms and in 
both cloud- and GSE-coordinates is to be included in the list of recent modifications to our MC fitting program, as well as those discussed in Sect. 2.3.

\subsection{Consideration of magnetic flux in clouds}

Panel (j) of Fig. 10 shows the distribution of axial magnetic fluxes, $\Phi_{0}$, for the 82 WIND MCs for the 8.6-year period of interest. When shown on a finer resolution the distribution shows a peak near $9 \times 10^{20} \mathrm{Mx}$ (or $9 \times 10^{10}$ Gauss $\mathrm{km}^{2}$ ); Table 2 shows an average value of about $10 \times 10^{20} \mathrm{Mx}$. There is obvious severe skewness in the $\Phi_{0}$-distributions. In Lepping and Berdichevsky $(2000)<\Phi_{0}>$ changed from about $13 \times 10^{20} \mathrm{Mx}$ for the "IMP" set of $18 \mathrm{MCs}(1967-1982)$ to $10 \times 10^{20} \mathrm{Mx}$ for the early WIND set of $28 \mathrm{MCs}$, consistent with our present value for the larger set of MCs. We should not be surprised that the more active period has the higher MC flux associated with it. We now make use of this information about a MCs typical axial magnetic flux in the following way, using the value of $10 \times 10^{10}$ Gauss $\mathrm{km}^{2}=\Phi_{0}(1 \mathrm{AU})$. We start by assuming that both ends of the MCs field lines are anchored at the Sun, at least for the period in which the $\mathrm{MC}$ transits from the Sun to $1 \mathrm{AU}$. If we assume complete axial flux conservation over $1 \mathrm{AU}$ and consider the value of $\Phi_{0}(1 \mathrm{AU})$ to be typical, and assume $2 \mathrm{R}_{S}=4 \times 10^{4} \mathrm{~km}$ to be a typical diameter of the MCs footprint at either end (see plate 2 of Martin and McAllister, 1997; Lepping et al., 1997b), then from

$\Phi_{0}(\operatorname{Sun})=\Phi_{0}(1 \mathrm{AU})$

or

$B_{S} \times$ Area $_{S}=B_{1} \times$ Area $_{1}$

$B_{S} \pi R_{S}^{2}=\mathrm{B}_{1} \pi \mathrm{R}_{1}^{2}=\Phi_{0}(1 \mathrm{AU})$,

where the subscript "S" refers to one of the footpoints at the Sun, and the subscript "1" refers to 1 AU. For the values of $\Phi_{0}(1 \mathrm{AU})$ and $\mathrm{R}_{S}$ given above, Eq. (12) yields a value for $\mathrm{B}_{S}$ of 80 Gauss. Such a value is not inconsistent with field intensities observed for prominence fields: values quoted for field intensities for quiescent prominences are around 5 to 30 Gauss (typically 8 Gauss), but for active region prominences at the foot of the flux tubes the field intensity has been estimated to be as high as 200 Gauss, but typically 20 to 70 Gauss are expected (Tandberg-Hanssen, 1995). So 80 Gauss is reasonably well bracketed by these values. This presumed connection of a typical MC to a solar source is therefore plausible, at least by this test. It is expected that the MCs footprint-fields will eventually disconnect, perhaps through magnetic merging. It might not occur until many days after the expulsion of the MC, but there has been insufficient study in this area to be more quantitative. Larson et al. (1997), however, have given solid evidence for at least partial field disconnection occurring, and a major change of field line topology, for the WIND MC of 18-20 October 1995 case (and see, e.g. Lepping et al., 1997a), even as early as the time when the MC reached $1 \mathrm{AU}$. Their work was based on an analysis of solar impulsive $\sim 10^{-1}-10^{2} \mathrm{keV}$ electron events measured within the MC using data from the WIND 3DP plasma instrument (Lin et al., 1995).

\section{Error estimations of magnetic cloud fit-parameters}

There are many possible sources of fit-parameter errors in modeling MCs. We list the most obvious ones here: (\#1) "noise" fluctuations in the IMF within the MC (meaning any non-MC-model perturbing fields), (\#2) incorrect choice of MC boundaries, (\#3) not accounting for systematic features in violation of the model (e.g. those due to MC expansion (Osherovich et al., 1993b; Berdichevsky et al., 2003), noncircular cross-section (Lepping et al., 1998), effects of a possible interaction of the MC with surrounding plasma, etc.), and (\#4) measurement errors in $\mathbf{B}$ and the velocity ( $\mathbf{V})$ of the MC. To these we could add the possible misidentification of a solar wind structure as a flux rope in the first place, but this may be considered to be included in category (\#2). We can generally ignore this source of error, since there are generally good means of providing assurance that the structure is a MC from the examination of the full plasma and field set together. Only the unusually short-duration cases present identification problems. But there is a possibility of incorrectly identifying combined MCs as a single MC, or the opposite, the incorrect identification of multiple MCs when the overall structure is really a single MC. We have taken pains to avoid such misidentifications, but can not guarantee that none have slipped through. In fact, we have discovered both kinds in our earlier list and corrected them. Category \#4 can almost always be ignored compared to source \#1; measurement errors are relatively very small. Modification of our model to account for the systematic features listed in source \#3 is planned for a later time. Source \#2 is usually important also, but we concentrated here only on \#1 as being the most tractable, and usually the most important, especially if care is taken to use all available means to obtain proper boundaries to solve uncertainty \#2.

If there were any doubt about the choice of the boundary positions, we would try many reasonable attempts at start/end times in the model parameter fitting, and then note the average and variation for the various output parameters, where the variations should reflect the degree of uncertainty due to this source of error. This error source may be considerable for what may be called "unstable" cases (where small estimated boundary changes give large changes in output parameter values), for which poor results are expected. The multiple trial method will not likely give a very accurate measure of this error, however, because we then do not usually have a quantitative measure of a "reasonable" attempt at start/end times. But there is usually no other choice, if the data set is restricted to a single spacecraft. (We should point out that the detailed features in the plasma parameters, especially proton temperature and proton beta, and even bulk speed (although not part of a MCs definition), are useful in choosing the proper boundaries. And the presence of field 
directional discontinuities is usually definitive in choosing boundaries, because a significant directional discontinuity (DD) should not exist within the MC, and therefore the closest DD may be the boundary. As mentioned above, sometimes a magnetic hole, i.e. a prominent dip in B (see, e.g. Burlaga, 1995; Farrugia et al., 2000; Baumgartel et al., 2003) will be seen at one or both boundaries of a MC.) If one of a series of boundary trials provided the highest Quality estimate (among $\mathrm{Q}_{0}=1,2,3$ ), that clearly would have been our choice. As stated above, source \#3 is beyond the scope of this study, but it is not completely independent of source \#1, because noise-fluctuations is a relative term, which, in fact, depends on the choice of model.

The model fit-parameters were recently examined in terms of level of fit-parameter uncertainty due to typical field fluctuation noise (source \#1), as seen generally in MCs. This is described in a recent fit-parameter error study by Lepping et al. (2003a, 2004) and implemented in a related program that interpolates between the output parameter values developed in the original Lepping et al. (1990) paper, to better enable its use. This error-interpolation program is given by the URL http://lepmfi.gsfc.nasa.gov/mfi/interpolation_mc.pro.

The Lepping et al. (2003a, 2004) fit-parameter error program was applied to the magnetic field within the interval of each of the identified 82 WIND MCs, and the interpolation process described above was employed. (See Appendix B, which provides some brief practical notes on background and use of the Lepping et al. (2003a, 2004) fit-parameter error program.) To summarize the results of the WIND fitparameter error study, Fig. 13 provides histograms of the parameter output uncertainties $(\sigma \mathrm{s})$ for the cases where $\mathrm{Q}_{0}$ is 1 or 2 , in the left column, and for $\mathrm{Q}_{0}=3$ in the right column, all for the following parameters: $\left|\theta_{A}\right|, \beta$ (cone), $\theta_{A}$, $\mathrm{CA}\left(=\mathrm{Y}_{0} / \mathrm{R}_{0}\right)$, asf $(\%), \mathrm{R}_{0}$, and $\mathrm{B}_{0}$, where the error-cone-angle $(\beta($ cone $))$ represents the statistically estimated angle between the exact $M C$ axis and the estimated MC axis; $\beta$ (cone) should not be confused with the angle $\beta_{C A}$, which is that angle between the estimated MC axis and $\mathbf{X}_{G S E}$ (see Eq. 3). Note that Handedness $(\mathrm{H})$ is not included, since $\mathrm{H}$ is always correct, unless the f-flag gives a NOT OK, as seen in Table 1. Table 5 gives the individual fit-parameter uncertainties $(\sigma \mathrm{s})$ for the specific MCs for the sets $\mathrm{Q}_{0}=1$ or 2 , with the premise that some of the parameter $\sigma \mathrm{s}$ for the $\mathrm{Q}_{0}=3$ cases are too poorly estimated to be quantitatively reliable, as the far right portions of most histograms in the right column of Fig. 13 (and their rmss) indicate. Specifically, the parameters given in Table 5 are: $\sigma \mathrm{B}_{0}, \sigma \mathrm{R}_{0}, \sigma$ asf $(\%), \sigma \mathrm{CA}\left(\equiv \sigma\left\{\mathrm{Y}_{0} \times 100 / \mathrm{R}_{0}\right\}\right)$, $\sigma \theta_{A}, \sigma \beta$ (cone), $\sigma\left|\theta_{A}\right|$, as well as $\mathrm{Q}_{0}$ just for comparison, and in that order.

The mean and rms values for each histogram in Fig. 13 are listed to the right of each panel. It is evident that the mean of $\sigma[\mathrm{X}]$ (where $\mathrm{X}$ is any one of $\mathrm{B}_{0}, \mathrm{R}_{0}$, etc.) is higher for all parameters for the $\mathrm{Q}_{0}=3$ cases with respect to the $\mathrm{Q}_{0}=1,2$ cases, but not always markedly so, and just barely so for $\sigma$ [asf]. However, for $\sigma$ [CA\%] there is a big difference between the two distributions and their means; CA is generally much more poorly determined for the $\mathrm{Q}_{0}=3$ cases.
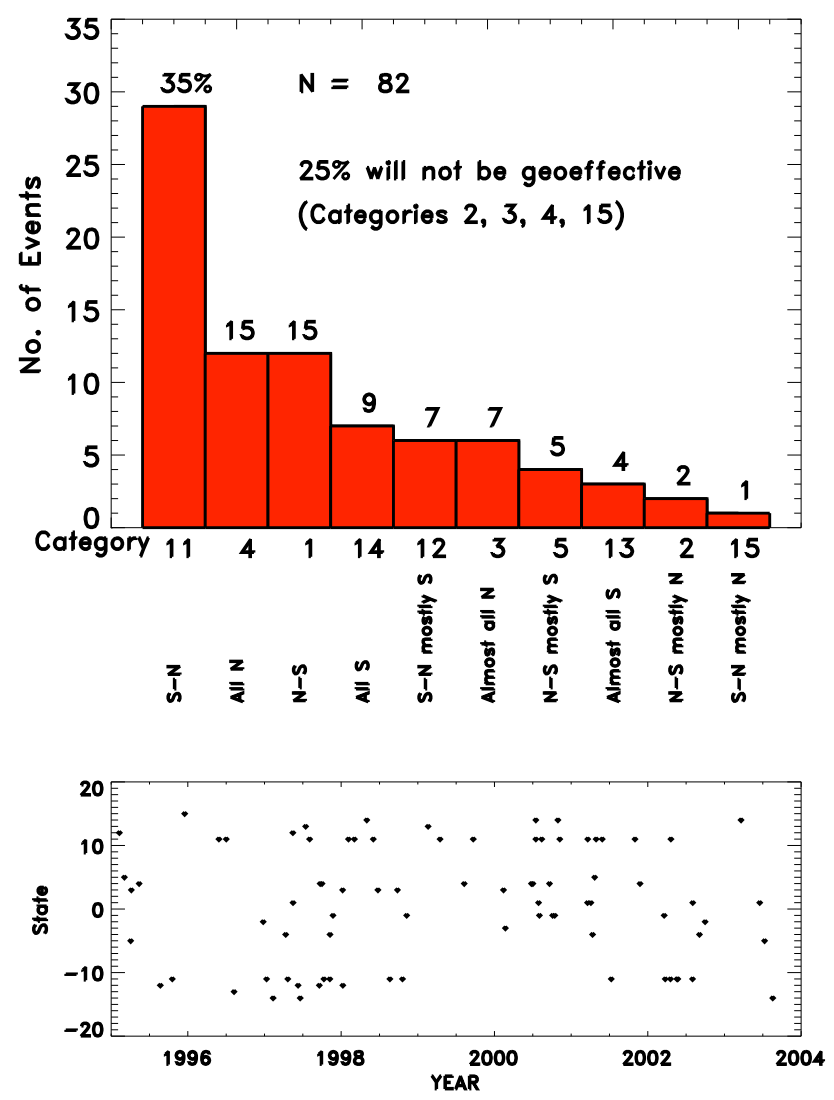

Fig. 12. Histogram of MCs separated into various Categories defined in Table 3 (from Lepping et al., 2005). Bottom: State of the MCs vs. time for the period from 1995 to August 2003, where State=Category H H (see Eq. (10) and text). See Table 3 for definitions of various MC categories. The State function takes into account handedness, axial polarity, and the attitude of the MC axis.

As was pointed out in Lepping et al. (2003a), CA is usually the most poorly determined parameter of all of the seven fit-parameters in the first place, so this big difference in the two distributions should not be surprising. At the bottom of Fig. 13 we also see a dramatic difference in the distributions and their means for $\sigma\left[\mathrm{B}_{0}\right]$ between the $\mathrm{Q}_{0}=3$ cases and the $\mathrm{Q}_{0}=1,2$ cases. Obviously for some $\mathrm{Q}_{0}=3$ cases $\mathrm{B}_{0}$ is quite poorly determined. Usually $\mathrm{B}_{0}$ is one of the best determined parameter for the $\mathrm{Q}_{0}=1$ or 2 cases.

\section{Magnetic cloud-like structures}

We developed a program to automatically identify MCs (Lepping et al., 2005). When applied to WIND data from launch to about August 2003, the program not only identified most $(\approx 88 \%)$ of those events that were previously identified as MFI-MCs, which we list in a Table 1 (called set 1 here), but it found many other similar events, which we designate magnetic cloud-like (MCL) structures. The full set found by the automatic identification program is called set 2 ; there are 185 events in this set. The structures in set 2 (sometimes 
Table 4. Closest approach vectors and magnetic cloud state values.

See footnotes at bottom of page 22 .

\begin{tabular}{|c|c|c|c|c|c|c|c|c|c|c|c|}
\hline \multicolumn{5}{|c|}{ Start Time ${ }^{a}$} & \multirow[b]{2}{*}{$Y_{0} / R_{0}(\%)^{b}$} & \multicolumn{2}{|c|}{$\mathrm{CA}\left(\right.$ unit) ${ }^{\mathrm{C}}$} & \multicolumn{2}{|c|}{$C A(G S E)^{d}$} & \multirow[b]{2}{*}{$Q_{0}{ }^{\ominus}$} & \multirow[b]{2}{*}{$s^{f}$} \\
\hline Yr & Mon & Day & Doy & $H R$ & & yy & $\mathbf{z z}$ & $Y(A U)$ & $Z(A U)$ & & \\
\hline 95 & 02 & 08 & 039 & 5.8 & -49 & 0.31 & -0.95 & 0.017 & -0.050 & 2 & -12 \\
\hline 95 & 03 & 04 & 063 & 10.8 & 49 & 0.99 & -0.10 & 0.040 & -0.004 & 3 & -5 \\
\hline 95 & 04 & 03 & 093 & 7.8 & -71 & -0.38 & -0.93 & -0.040 & -0.100 & 2 & 5 \\
\hline 95 & 04 & 06 & 096 & 7.3 & -81 & 0.95 & -0.31 & 0.032 & -0.010 & 2 & -3 \\
\hline 95 & 05 & 13 & 133 & 10.9 & -112 & -0.47 & -0.88 & -0.025 & -0.047 & 3 & -4 \\
\hline 95 & 08 & 22 & 234 & 21.3 & -48 & -0.03 & 1.00 & -0.002 & 0.060 & 2 & 12 \\
\hline 95 & 10 & 18 & 291 & 19.8 & 12 & 0.15 & -0.99 & 0.002 & -0.015 & 1 & 11 \\
\hline 95 & 12 & 16 & 350 & 5.3 & -84 & -1.00 & -0.00 & -0.048 & -0.000 & 3 & -15 \\
\hline 96 & 05 & 27 & 148 & 15.3 & 11 & -0.59 & 0.81 & -0.011 & 0.016 & 2 & -11 \\
\hline 96 & 07 & 01 & 183 & 17.3 & 16 & -0.10 & 0.99 & -0.001 & 0.014 & 2 & -11 \\
\hline 96 & 08 & 07 & 220 & 12.3 & 46 & 0.81 & -0.58 & 0.040 & -0.029 & 1 & 13 \\
\hline 96 & 12 & 24 & 359 & 2.8 & -47 & 0.44 & -0.90 & 0.030 & -0.060 & 1 & 2 \\
\hline 97 & 01 & 10 & 010 & 5.3 & -11 & 0.02 & 1.00 & 0.000 & 0.010 & 1 & 11 \\
\hline 97 & 02 & 10 & 041 & 3.4 & 120 & -0.27 & 0.96 & -0.039 & 0.139 & 3 & 14 \\
\hline 97 & 04 & 11 & 101 & 5.6 & -40 & 0.90 & -0.44 & 0.047 & -0.023 & 2 & 4 \\
\hline 97 & 04 & 21 & 111 & 14.5 & -23 & -0.18 & 0.98 & -0.003 & 0.016 & 3 & 11 \\
\hline 97 & 05 & 15 & 135 & 9.1 & 24 & 0.00 & 1.00 & 0.000 & 0.022 & 2 & -12 \\
\hline 97 & 05 & 16 & 136 & 6.1 & 45 & -0.66 & -0.75 & -0.011 & -0.012 & 3 & -1 \\
\hline 97 & 06 & 09 & 160 & 2.3 & 53 & 0.56 & -0.83 & 0.027 & -0.041 & 2 & 12 \\
\hline 97 & 06 & 19 & 170 & 5.1 & -15 & -0.34 & 0.94 & -0.003 & 0.009 & 3 & 14 \\
\hline 97 & 07 & 15 & 196 & 8.8 & 0 & N/A & N/A & 0.000 & 0.000 & 3 & -13 \\
\hline 97 & 08 & 03 & 215 & 14.1 & 83 & -0.21 & 0.98 & -0.005 & 0.024 & 3 & -11 \\
\hline 97 & 09 & 18 & 261 & 0.5 & -61 & 0.89 & 0.46 & 0.090 & 0.046 & 3 & 12 \\
\hline 97 & 09 & 22 & 265 & 0.8 & 3 & -0.96 & 0.27 & -0.003 & 0.001 & 2 & -4 \\
\hline 97 & 10 & 01 & 274 & 16.3 & -44 & 0.71 & -0.70 & 0.062 & -0.061 & 2 & -4 \\
\hline 97 & 10 & 10 & 283 & 23.8 & -57 & -0.03 & 1.00 & -0.002 & 0.065 & 1 & 11 \\
\hline 97 & 11 & 07 & 311 & 15.8 & -16 & 0.66 & 0.75 & 0.012 & 0.013 & 2 & 11 \\
\hline 97 & 11 & 08 & 312 & 4.9 & -48 & 0.99 & 0.12 & 0.027 & 0.003 & 2 & 4 \\
\hline 97 & 11 & 22 & 326 & 15.8 & -23 & -0.81 & 0.59 & -0.029 & 0.021 & 3 & 1 \\
\hline 98 & 01 & 07 & 007 & 3.3 & -2 & 0.96 & -0.29 & 0.002 & -0.001 & 1 & -3 \\
\hline 98 & 01 & 08 & 008 & 14.9 & -39 & 0.86 & 0.51 & 0.018 & 0.010 & 3 & 12 \\
\hline 98 & 02 & 04 & 035 & 4.5 & -89 & 0.14 & -0.99 & 0.018 & -0.130 & 2 & -11 \\
\hline 98 & 03 & 04 & 063 & 14.3 & -6 & 0.41 & -0.91 & 0.004 & -0.009 & 1 & -11 \\
\hline 98 & 05 & 02 & 122 & 12.3 & -83 & 1.00 & -0.01 & 0.191 & -0.002 & 3 & -14 \\
\hline
\end{tabular}

called the "joint" set) should at least resemble actual MCs, since the "automatic" program uses criteria that were based on MC plasma and field characteristics determined from the study of the earlier visually identified cases of MCs, i.e. set 1 , in the first place. Strictly speaking, then the MCL set (called set 3 here) is defined as the difference-set (set 2-set 1), and it is even bigger than set $1\left(\mathrm{~N}_{\mathrm{MFI}}=103\right)$. As Fig. 14 shows, the occurrence frequency of the joint set (set 2), in fact, better matches that of the sunspot cycle than set 1 , obviously indicating the joint set's better association with ejecting so- lar sources than set 1 . However, many cases in the MCL set are less intense (i.e. lower $<\mathrm{B}>$ ) and have relatively shorter durations than those in set 1 , and rarely do they represent good flux ropes through force free parameter modeling. Nevertheless, many of the MCL structures are geoeffective having long periods of negative $\mathrm{B}_{z}$, being somewhat similar to most bona fide MCs in that respect. Some of their unusual characteristics may be the result of distant spacecraft passages (with respect to the MC axes) of actual MCs, but some may be non-MC ejecta. The start and end times of 
Table 4. Continued.

See footnotes at bottom of page 22 .

\begin{tabular}{|c|c|c|c|c|c|c|c|c|c|c|c|}
\hline \multicolumn{5}{|c|}{ Start Time a } & \multirow[b]{2}{*}{$Y_{0} / R o(\%)^{b}$} & \multicolumn{2}{|c|}{$C A\left(\right.$ unit) ${ }^{c}$} & \multicolumn{2}{|c|}{$C A(G S E)^{d}$} & \multirow[b]{2}{*}{$Q_{0}^{\theta}$} & \multirow[b]{2}{*}{$s^{f}$} \\
\hline$\overline{Y r}$ & Mon & Day & Doy & $\overline{H R}$ & & yy & 22 & $Y(A U)$ & $Z(A U)$ & & \\
\hline 98 & 06 & 02 & 153 & 10.6 & 20 & -0.60 & 0.80 & -0.004 & 0.006 & 2 & -11 \\
\hline 98 & 06 & 24 & 175 & 16.8 & -23 & 0.62 & -0.78 & 0.015 & -0.019 & 2 & -3 \\
\hline 98 & 08 & 20 & 232 & 10.3 & -13 & 0.32 & 0.95 & 0.004 & 0.013 & 1 & 11 \\
\hline 98 & 09 & 25 & 268 & 10.3 & 57 & -0.89 & -0.46 & -0.104 & -0.053 & 2 & -3 \\
\hline 98 & 10 & 19 & 292 & 5.1 & 93 & -1.00 & -0.10 & -0.019 & -0.002 & 3 & 11 \\
\hline 98 & 11 & 08 & 312 & 23.8 & 16 & 0.96 & 0.27 & 0.019 & 0.005 & 1 & 1 \\
\hline 99 & 02 & 18 & 049 & 14.3 & -63 & 0.14 & 0.99 & 0.023 & 0.158 & 3 & -13 \\
\hline 99 & 04 & 16 & 106 & 20.3 & 46 & 0.45 & 0.89 & 0.022 & 0.042 & 3 & -11 \\
\hline 99 & 08 & 09 & 221 & 10.8 & 26 & -1.00 & 0.03 & -0.035 & 0.001 & 1 & -4 \\
\hline 99 & 09 & 21 & 264 & 21.1 & 63 & -0.10 & 0.99 & -0.003 & 0.028 & 3 & -11 \\
\hline 00 & 02 & 12 & 043 & 17.1 & 33 & 0.67 & -0.75 & 0.008 & -0.009 & 3 & -3 \\
\hline 00 & 02 & 21 & 052 & 9.8 & -22 & 1.00 & -0.05 & 0.029 & -0.002 & 3 & 3 \\
\hline 00 & 06 & 24 & 176 & 8.3 & -127 & 0.74 & -0.67 & 0.278 & -0.251 & 3 & -4 \\
\hline 00 & 07 & 01 & 183 & 8.8 & -47 & 0.93 & 0.36 & 0.058 & 0.022 & 1 & -4 \\
\hline 00 & 07 & 15 & 197 & 6.8 & -95 & -0.99 & -0.14 & -0.084 & -0.012 & 2 & -14 \\
\hline 00 & 07 & 15 & 197 & 21.1 & -17 & 0.82 & -0.57 & 0.022 & -0.016 & 2 & -11 \\
\hline 00 & 07 & 28 & 210 & 21.1 & -53 & -0.21 & 0.98 & -0.013 & 0.059 & 2 & -1 \\
\hline 00 & 08 & 01 & 214 & 0.1 & -82 & -0.18 & 0.98 & -0.015 & 0.081 & 3 & 1 \\
\hline 00 & 08 & 12 & 225 & 6.1 & -1 & 0.05 & -1.00 & 0.000 & -0.001 & 2 & -11 \\
\hline 00 & 09 & 18 & 262 & 1.9 & 116 & 0.99 & -0.17 & 0.103 & -0.018 & 3 & -4 \\
\hline 00 & 10 & 03 & 277 & 17.1 & 23 & -0.61 & 0.79 & -0.013 & 0.017 & 1 & 1 \\
\hline 00 & 10 & 13 & 287 & 18.4 & 11 & 0.80 & 0.60 & 0.011 & 0.008 & 2 & 1 \\
\hline 00 & 10 & 28 & 302 & 23.3 & 20 & -0.44 & 0.90 & -0.023 & 0.048 & 3 & -14 \\
\hline 00 & 11 & 06 & 311 & 23.1 & -19 & -0.17 & -0.99 & -0.004 & -0.026 & 2 & -11 \\
\hline 01 & 03 & 19 & 078 & 23.3 & -19 & -0.78 & 0.63 & -0.012 & 0.010 & 1 & -1 \\
\hline 01 & 03 & 20 & 079 & 17.8 & -4 & -0.50 & -0.87 & -0.005 & -0.008 & 3 & -11 \\
\hline 01 & 04 & 04 & 094 & 20.9 & -86 & 0.14 & 0.99 & 0.023 & 0.162 & 1 & -1 \\
\hline 01 & 04 & 12 & 102 & 7.9 & 68 & -0.82 & -0.58 & -0.069 & -0.049 & 2 & 4 \\
\hline 01 & 04 & 22 & 112 & 0.9 & 5 & 0.98 & -0.19 & 0.007 & -0.001 & 2 & -5 \\
\hline 01 & 04 & 29 & 119 & 1.9 & 39 & -0.57 & 0.82 & -0.026 & 0.037 & 2 & -11 \\
\hline 01 & 05 & 28 & 148 & 11.9 & 37 & 0.27 & 0.96 & 0.013 & 0.045 & 1 & -11 \\
\hline 01 & 07 & 10 & 191 & 17.3 & -51 & 0.17 & 0.99 & 0.011 & 0.064 & 2 & 11 \\
\hline 01 & 10 & 31 & 304 & 21.3 & -9 & -0.05 & -1.00 & -0.001 & -0.013 & 3 & -11 \\
\hline 01 & 11 & 24 & 328 & 15.8 & -80 & 0.52 & -0.85 & 0.118 & -0.191 & 3 & -4 \\
\hline
\end{tabular}

the events in set 3 (MCL set) can be found at the URL of http://lepmfi.gsfc.nasa.gov/mfi/MCL1.html.

Plans are to examine these against other lists of ejecta, including ICMEs, the interplanetary counterpart to coronal mass ejections (see, e.g. Kahler, 1987; Gosling, 1990, 1997).

With some modifications the Lepping et al. (2005) program, now used for automatically and objectively identifying MCs generally, may also be used in a real-time mode for predicting the point where IMF- $\mathrm{B}_{z}$ goes to a minimum late in a $\mathrm{MC}$ from information within the early part of the MC for $N \rightarrow S$ cases. These are expected to be the most probable type of MCs in the near future (starting about year 2007), as discussed by Lepping et al. (2005). [And see Bothmer and Rust (1997), concerning the field configurations of MCs with respect to the solar cycle, and Chen et al. (1997), who also developed a scheme for predicting solar wind structures and degree of their geoeffectiveness, based on solar wind measurements made immediately prior to the prediction time, but with no special concern for whether they were MCs or not.] With such real-time information, forecasting of minimum 
Table 4. Continued.

\begin{tabular}{|c|c|c|c|c|c|c|c|c|c|c|c|}
\hline \multicolumn{5}{|c|}{ Start Time ${ }^{a}$} & \multirow[b]{2}{*}{$Y_{0} / R o(\%)^{b}$} & \multicolumn{2}{|c|}{$\mathrm{CA}$ (unit) $^{\mathrm{C}}$} & \multicolumn{2}{|c|}{$C A(G S E)^{d}$} & \multirow[b]{2}{*}{$Q_{0}^{\theta}$} & \multirow[b]{2}{*}{$s^{f}$} \\
\hline Yr & Mon & Day & Doy & HR & & yy & 22 & $Y(A U)$ & $Z(A U)$ & & \\
\hline 02 & 03 & 19 & 078 & 22.9 & -18 & 0.46 & -0.89 & 0.009 & -0.017 & 2 & 1 \\
\hline 02 & 03 & 24 & 083 & 3.8 & 8 & -0.59 & -0.81 & -0.010 & -0.014 & 2 & 11 \\
\hline 02 & 04 & 18 & 108 & 4.3 & -53 & -0.61 & 0.80 & -0.051 & 0.067 & 1 & 11 \\
\hline 02 & 04 & 20 & 110 & 11.8 & 44 & -0.74 & 0.67 & -0.047 & 0.043 & 3 & -11 \\
\hline 02 & 05 & 19 & 139 & 3.9 & -95 & 0.04 & 1.00 & 0.007 & 0.201 & 1 & 11 \\
\hline 02 & 05 & 23 & 143 & 23.4 & -113 & 0.70 & 0.72 & 0.244 & 0.252 & 3 & 11 \\
\hline 02 & 08 & 01 & 213 & 11.9 & -25 & 0.82 & 0.57 & 0.014 & 0.009 & 3 & 11 \\
\hline 02 & 08 & 02 & 214 & 7.4 & 11 & 0.17 & -0.99 & 0.002 & -0.014 & 2 & -1 \\
\hline 02 & 09 & 03 & 246 & 0.3 & 36 & -0.87 & -0.49 & -0.023 & -0.013 & 2 & 4 \\
\hline 02 & 09 & 30 & 273 & 22.6 & -10 & -0.29 & -0.96 & -0.002 & -0.006 & 3 & 2 \\
\hline 03 & 03 & 20 & 079 & 11.9 & 66 & 0.99 & -0.13 & 0.068 & -0.009 & 1 & -14 \\
\hline 03 & 06 & 17 & 168 & 17.8 & -17 & -0.79 & 0.61 & -0.017 & 0.014 & 3 & -1 \\
\hline 03 & 07 & 10 & 191 & 19.9 & -54 & -0.89 & -0.45 & -0.047 & -0.023 & 3 & 5 \\
\hline 03 & 08 & 18 & 230 & 11.6 & -12 & -0.80 & 0.60 & -0.014 & 0.010 & 2 & 14 \\
\hline
\end{tabular}

\section{Footnotes :}

a Start time in Year, Month, Day of Month, Day of Year, Hour

$b Y_{0} / R_{0}$ (in \%) (a scalar quantity); this is along the $Y$-axis in Cloud coordinates

c CA (unit) is $Y_{0} / \mid Y_{0} l$, a unit vector, in GSE coordinates, where the $X$-component $(x x)$ is zero

d CA (GSE) is $Y_{0}$ expressed in GSE coordinates, in units of AU

e Quality, Qo

$f$ State is defined by $\mathrm{Eq}(10)$ [State $=$ Category $\times \mathrm{H}$, where $\mathrm{H}$ is handedness and Category is defined in Table 3 (e.g., $N \rightarrow$ or $S \rightarrow N$, etc.)]

$D_{s t}$ (and its point of occurrence), for the expected type of $\mathrm{N} \rightarrow \mathrm{S} \mathrm{MCs}$, is possible many hours before it occurs, because of the large size of most MCs and also because of the approximately 1-h lead-time from spacecraft at L1 to Earth, such as provided by ACE and WIND (presently).

\section{Some particular properties of magnetic clouds}

In this section we review some findings by the WIND MFI team in their general and specific examinations of WIND MCs either not given above or given only briefly. A few items are a review of important properties determined by others, for completeness.

\subsection{Particular properties of magnetic clouds}

At 1 AU $56 \%$ of MCs drove interplanetary shocks, i.e. 46 out of 82 MCs (if we include 5 upstream pressure pulses apparently caused by the MC as driver and appearing as shocks in formation) (Lepping et al., 2002). And 10\% of the total MCs had an interplanetary shock inside (e.g. Lepping et al., 1997a; Collier et al., 2001, 2005); it is not yet clear why so many MCs have these internal shocks.

There were two very short duration MCs: both were estimated to be small: 1) the MC of 13 May 1995 with a $R_{0}$ of
0.048 and duration of $5.5 \mathrm{~h}$ (with $\mathrm{Q}_{0}$ of 3 ), and 2) the MC of 2 June 1998 with an $R_{0}$ of 0.035 and duration of $5.3 \mathrm{~h}$ (with $\mathrm{Q}_{0}$ of 2). Some others with short durations did not necessarily have small $\mathrm{R}_{0}$ s, such as the MC of 20 March 2003 with an $\mathrm{R}_{0}$ of 0.101 and duration of $10.5 \mathrm{~h}$, but the $|\mathrm{CA}|$ was $70 \%$ and the speed was $650 \mathrm{~km} / \mathrm{s}$ (with a $\mathrm{Q}_{0}$ of 1 ). There are other good examples of small flux ropes in the solar wind (e.g. Moldwin et al., 1999) that are apparently not MCs for many reasons, as argued by Moldwin et al.

There were at least 4 MCs with a core-annulus structure (as defined by right side of Fig. 6), occurring on: 1) 18 September $1997\left(\mathrm{Q}_{0}=3\right)$, 2) 4 February $1998\left(\mathrm{Q}_{0}=2\right)$, already discussed (see Fig. 8), 3) 8 November $1998\left(\mathrm{Q}_{0}=1\right)$, and 4) 24 June $2000\left(Q_{0}=3\right)$. Actually case (3) appears to be an annulus-only case. These four cases are marked by an * in the ck\% column of Table 1; often the ck\% value for such cases is very large, although not among the largest of such values, which are explained in other ways.

Any specific MC may be part of multiple or complex ejecta (Burlaga et al., 2002; Farrugia and Berdichevsky, 2004). There was no attempt to address this issue here, and likely some of our MCs were, indeed, part of such complexes. 

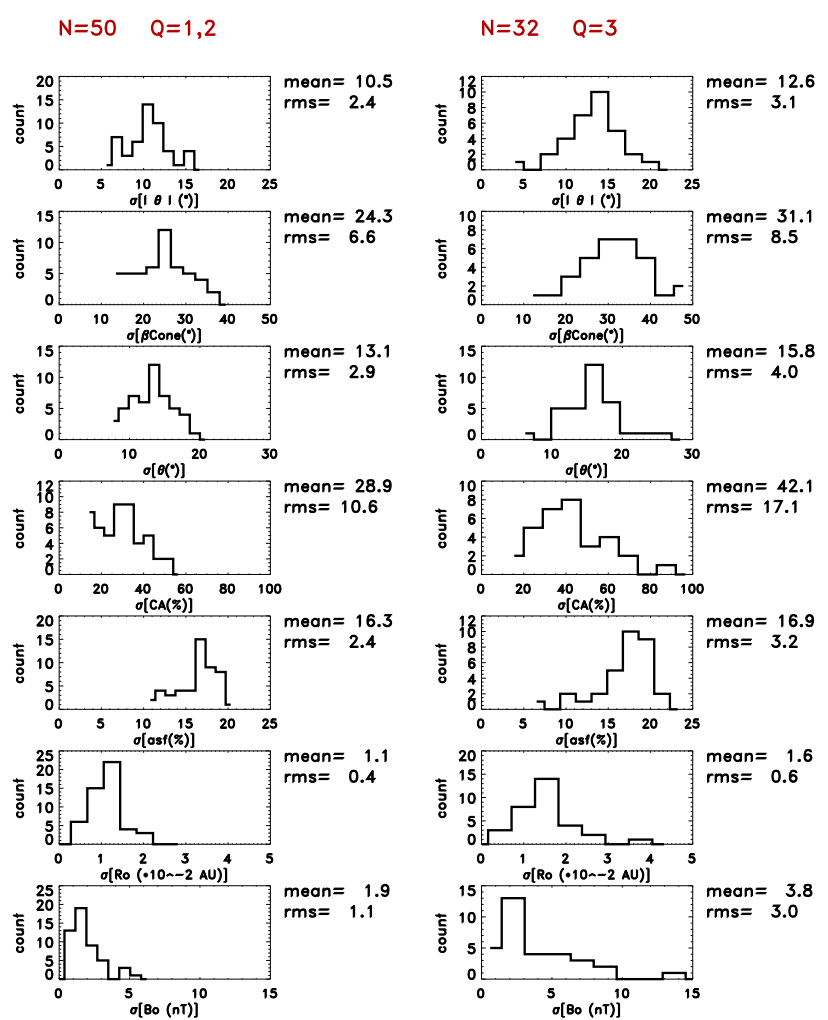

Fig. 13. Histograms of the uncertainties $(\sigma \mathrm{s})$ for various MC parameters (in the order $\left|\theta_{A}\right|, \beta_{C A}, \theta_{A}, \mathrm{CA}\left(=\mathrm{Y}_{0} / \mathrm{R}_{0}\right.$, in $\left.\%\right)$, asf(in $\%), \mathrm{R}_{0}$, and $\left.\mathrm{B}_{0}\right)$, where the combined $\mathrm{Q}_{0}=1,2$ cases $(\mathrm{N}=50)$ are in the first column and the $\mathrm{Q}_{0}=3$ cases $(\mathrm{N}=32)$ are in the second column, as noted. For any given case the designations of mean and rms hold for the specific histogram shown to their left, and they have the same units as that histogram, as shown below it. An rms for a given histogram provides a measure of the spread in the parameter uncertainties across a particular set of MCs; as expected, the $\mathrm{Q}_{0}=3$ cases show larger rmss than the $\mathrm{Q}_{0}=1,2$ cases.

Unusual composition is observed in MCs, as in ICMEs (e.g. Steinberg et al. (1997) where often $\mathrm{He}^{++}$enhancements were observed). Also see McComas et al. (1998) on ACE measurements of composition with respect to CMEs.

There is a fairly high incidence of MCs occurring at sector boundaries (Crooker and Intriligator, 1996; Crooker et al., 1998a,b), at least as measured by the high incidence of near $180^{\circ}$ changes in $\phi_{B}$ in the WIND magnetic field data. These authors cogently explain the reason for this high correlation between MCs and sector boundaries.

The frequency of occurrence of bona fide WIND MCs is given by the diamonds in the lower part of Fig. 14. They clearly do not correlate well with the Sunspot number (given by triangles in Fig. 14). But the joint set (given by xxx, and comprised of WIND MCs plus the automatically found set, MCLs, resembling actual MCs) fairly well correlates with Sunspot number, with a correlation coefficient of 0.80 . This must be investigated further.

Relatively uniform speed decreases across most MCs (and associated MC-expansions) at $1 \mathrm{AU}$ are well known. In the

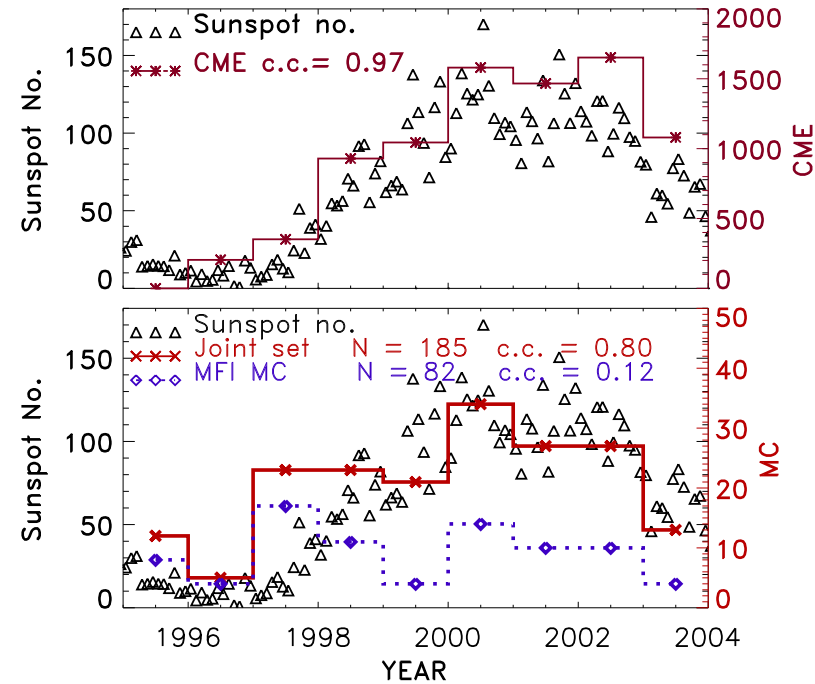

Fig. 14. (Top) Monthly sunspot number (SSN, triangles) and number of CMEs (***) (Gopalswamy et al., 1998) vs. time over the period of 1995 through 2003; the CMEs are yearly-averaged. The CMEs very well track the SSNs, as expected with a linear correlation coefficient, c.c. $=0.97$. (Bottom) SSN (triangles) vs. time, and yearly averaged occurrence of WIND MCs (dots-diamonds) and joint set (MC+MCL) of events (solid-xs) vs. time for the same time period; MCL refers to magnetic cloud-like structures that are determined by a program developed by Lepping et al. (2005) (see Sections 2.1 and 6.0). In this panel it is evident that the joint set much better correlates with SSN than with the (MFI) MC set alone. In fact, the linear correlation coefficient for WIND MCs vs. SSN was only c.c. $=0.12$, but for MCLs vs. SSN it was c.c. $=0.80$.

WIND set of MCs $76 \%$ had such uniform speed decreases, if 9 (out of the full 82) borderline cases are included; here a borderline case means the speed decreased across the MC but not necessarily in a very uniform way. Hence, at least to $1 \mathrm{AU}$ most MCs are expanding, and probably many do so well beyond $1 \mathrm{AU}$ (e.g. Skoug et al., 2000).

As exemplified by Fig. 4, magnetic holes are sometimes observed at MC boundaries usually helping in the determination of the MC boundary or boundaries (e.g. Burlaga, 1995, and Farrugia et al., 2000). In the WIND set a preliminary examination finds that in about $60 \%$ of all cases there is a magnetic hole at either the front or rear boundary or both, even if they were sometimes shallow or somewhat broad in appearance. In other words, 31 cases clearly had no magnetic holes, front or rear; a plasma data gap in one case did not allow any determination. Few cases were as clear as those holes shown in Fig. 4.

There is a strong tendency for the handedness $(\mathrm{H})$ of the field in a magnetic cloud to be correlated with a given hemisphere of the Sun, independent of solar cycle, with left-handed MCs being associated with the Northern Hemisphere and right-handed with the Southern Hemisphere, just as found for solar filamentary structure (Rust,1994 and references therein; also see Rust and Kumar, 1994; and Bothmer, 2003). 


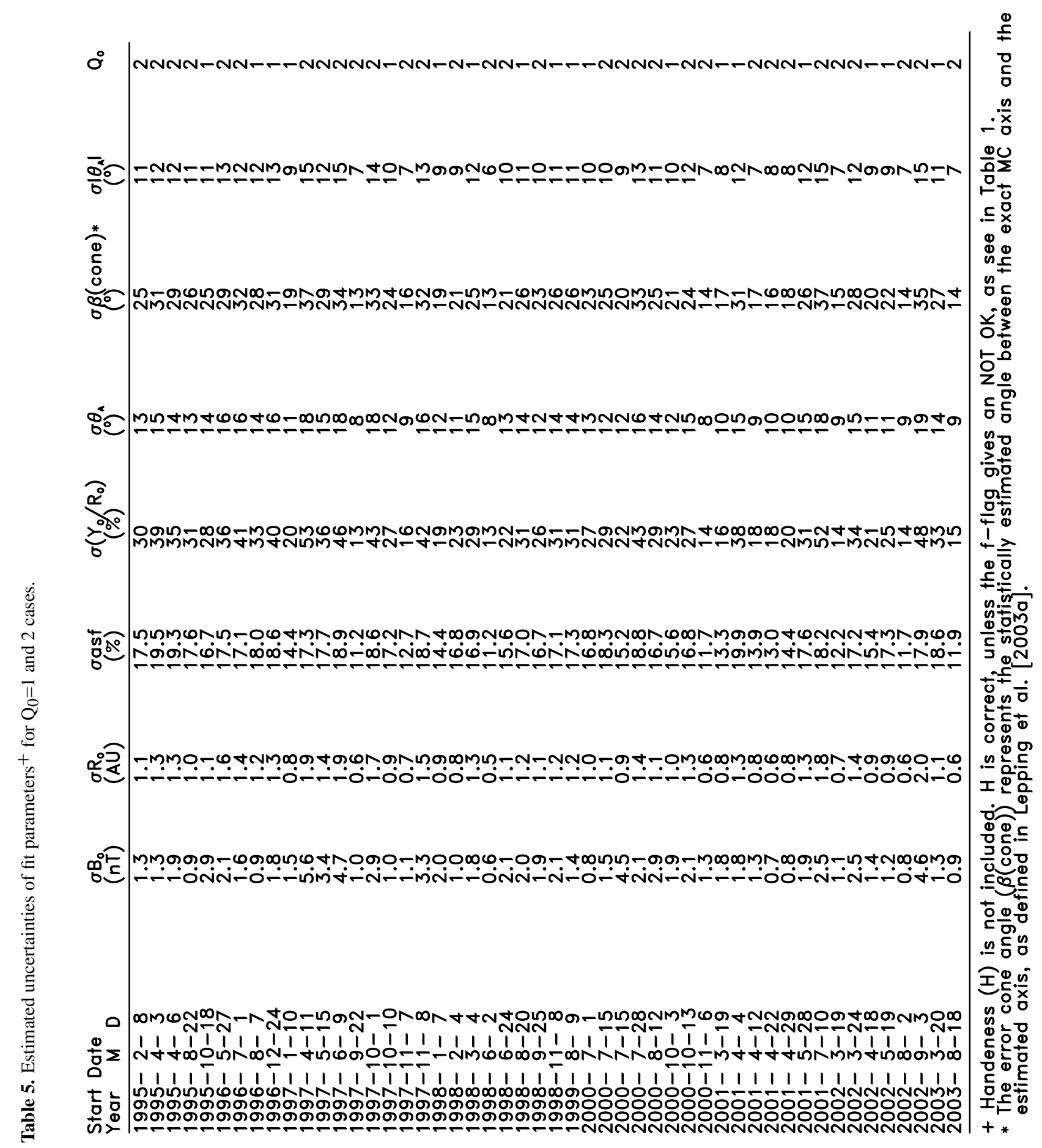


7.2 What MC parameters tend to be approximately invariant at $1 \mathrm{AU}$ ?

We ask, is there a tendency for the Sun to produce MCs with a narrow range of $\mathrm{B}_{0} \mathrm{~s}$, or diameters, and/or other parameters (e.g. total axial current $\left(\mathrm{I}_{T}\right), \mathrm{J}_{0}, \Phi_{0}$, and $\mathrm{B}_{0}^{2} / 8 \pi$ ), as measured by $\sigma /$ Mean and $\sigma$ Median/Median (see footnote of Table 6A for a definition), which are relative standard deviations (r.s.d.s)? And further, what is the specific values of these ratios for each parameter for a large number of cases? In Tables 6A, B we show computed values for these ratios for these particular $\mathrm{MC}$ parameters $\left(\mathrm{B}_{0}\right.$, Diameter, etc.) for the full set of $\mathrm{N}=82 \mathrm{MCs}$ (in Table 6A) and for the best cases, where $\mathrm{Q}_{0}=1,2$ (in Table $6 \mathrm{~B}$ ), both showing a clear trend among the parameters. The trends for the two different ratios is the same within the full set and similar for the $\mathrm{Q}_{0}=1,2$ set. For the apparently more reliable $\mathrm{Q}_{0}=1,2$ set, it is clear that the r.s.d.s for $\mathrm{B}_{0}$ and diameter are rather narrow, but for $\mathrm{B}_{0}^{2} / 8 \pi$ and $\Phi_{0}$ they are broad, and for total axial current $\left(\mathrm{I}_{T}\right)$ and $\mathrm{J}_{0}$ they are intermediate in value. Hence, there is a tendency for the Sun to keep the axial magnetic field and size of a MC more invariant than the MCs axial- $\Phi_{0}$, and/or $\mathrm{B}_{0}^{2} / 8 \pi$ (with total current and $\mathrm{J}_{0}$ as intermediate cases) - based on our model (Lepping et al., 1990) for the 8.6-years period of interest. Some of the reason for this is obviously due to the manner in which parameter errors propagate.

\section{Summary and discussion}

MCs usually have the field structure of very large magnetic flux ropes (with average durations of $21 \mathrm{~h}$ ), with strong magnetic fields, and containing relatively cool internal proton plasma. All of the many MCs considered here $(\mathrm{N}=82)$ were analyzed according to a relatively simple static force free cylindrically symmetric MC model (Lepping et al., 1990). The relevant seven model-parameters are presented in Sect. 2.2. Section 2.3 describes recent modifications to the program to help assess the "quality" $\left(\mathrm{Q}_{0}\right)$ of the models parameter-fitting for any MC; also a scheme was developed to estimate the uncertainties on the fit-parameters, which was implemented for the good or fair quality cases. Examples of how boundaries are chosen were given, and there was discussion of the difficulties in determined these boundaries, especially when there are apparent inconsistencies between requirements of the MC fit model (for a given magnetic field data set) and the plasma data, such as when indicated by proton temperature, plasma $\beta$, and speed gradient. Examples of MC parameter fitting to the magnetic field, according to the model of Lepping et al. (1990) (given by red dashed curves), are shown in Figs. 1, 3, 4, 7, 8, and 9. Table 1 gives the overall summary of the basic fit-parameters for all 82 cases.

A by-product of the modeling is its ability to estimate a MC's axial magnetic flux $\left(\Phi_{0}\right)$, the axial current density $\left(\mathbf{J}_{0}\right)$, and total axial current $\left(\mathrm{I}_{T}\right)$, carried by a $\mathrm{MC}$ at the observing spacecraft's distance from the Sun. All such estimates have been made for WIND, and a comprehensive set of various
MC derived parameter values, including these by-products, and $\mathrm{Q}_{0}$, are also shown in Table 1 . We have extrapolated the axial magnetic flux back to the Sun, for a few observed MCs, obtaining acceptable consistency with the expected values of the flux of the solar magnetic arcades in the lower corona (Lepping et al., 1997b).

Table 2 and Figs. 10 and 11 give a comprehensive overview of our MC modeling results in terms of parameter distributions. We here summarize a few basic determined properties: on average the WIND MCs are just under one day long, are 1/4 AU in diameter, have a broad distribution of axial directions with a slight preference for alignment with the Y-axis(GSE), have axial fluxes of $10^{21} \mathrm{Mx}$, have axial current densities of about $2 \mu \mathrm{A} / \mathrm{km}^{2}$, and carry a total axial current $\left(\mathrm{I}_{T}\right)$ of about a billion amps. Their speeds were typically around $400 \mathrm{~km} / \mathrm{s}$ for the first four years of WINDs mission (Lepping and Berdichevsky, 2000), but they have climbed to significantly larger values recently with some values between 600 and $800 \mathrm{~km} / \mathrm{s}$; the 8.6 -year-average is $\langle\mathrm{V}\rangle=450 \mathrm{~km} / \mathrm{s}$. Some of these values are expected to change as the MC is observed at distances other than at $1 \mathrm{AU}$, as indeed, they have been observed to do (Skoug et al., 2000). Table 5 provides uncertainty estimates for the $\mathrm{Q}_{0}=1,2 \mathrm{MCs}$ for many of the key MC fit-parameters of Table 1 based on a Monte Carlo scheme (Lepping et al., 2003a, 2004). Model MC parameteruncertainties are summarized in histograms for $\mathrm{N}=82$ cases separated according to $\mathrm{Q}_{0}$-values in Fig. 13.

We classified the full set of MCs not only according to $\mathrm{Q}_{0}$, but also by Category (i.e. profiles of $\mathrm{S} \rightarrow \mathrm{N}, \mathrm{N} \rightarrow \mathrm{S}$, etc.; see Table 3) and State (see Eq. (10) and Fig. 12), which requires knowledge of $\mathrm{H}$. (For a comprehensive understanding of MCs in general, State is important, among other physical quantities.) However, for geomagnetic storm forecasting, knowing Category, speed, and density are crucial, but knowing State is less important. For example, for an $\mathrm{N} \rightarrow \mathrm{S}$ case (Category 1), we can predict $\mathrm{B}_{z}$ in the latter part of the MC, where $\mathrm{B}_{z}$ is mostly southward, based on observations from the earlier part of the MC, in order to then forecast the resulting storm $D_{s t}$ and its timing (e.g. Lepping et al., 2005). Hence, knowing the particular Category of a MC may aid in carrying out $D_{s t}$ forecasting. In summary, we have classified MCs according to $\mathrm{Q}_{0}$, Category, and State, each having its own purpose. But for study of the expansion-field of a $\mathrm{MC}$, classification according to $\mathrm{CA}\left(=\left|\mathbf{Y}_{0}\right| / \mathrm{R}_{0}\right)$ may also be necessary, since the plasma radial speed in the MC frame of reference should depend on the spacecrafts CA (measured from the $\mathrm{MC}$ axis) at which the plasma speed was measured in the MC frame of reference; this frame of reference is described in the end of Sect. 2.2. Closest approach vectors of the observing spacecraft for the full set of MCs are given in Table 4; these may be especially useful for solar studies.

Recently we developed a program to automatically identify MCs (Lepping et al., 2005). When applied to the first 8.6 years of WIND data the program identified $88 \%$ of those events that were previously identified through visual inspection as MCs. It also found many other similar events, i.e. "false positives", which we designate magnetic cloud-like 
Table 6A. Parameter variation measured by $\sigma /$ Mean and $\sigma$ Median/Median for all 82 cases.

\begin{tabular}{lrrrrrrr} 
Quantity & Mean & Median & \multicolumn{1}{c}{$\sigma$} & $\sigma$ Median & $\sigma /$ Mean & $\sigma$ Median/Med & Trend \\
\hline $\mathrm{B}_{0}(\mathrm{nT})$ & 17.8 & 16.6 & 6.87 & 6.97 & 0.386 & 0.420 & $\downarrow$ \\
Diameter $(\mathrm{AU})$ & 0.249 & 0.238 & 0.122 & 0.123 & 0.491 & 0.516 & $\downarrow$ \\
$\mathrm{I}_{\mathrm{T}}\left(10^{8} \mathrm{Amps}\right)$ & 8.57 & 7.05 & 5.71 & 5.92 & 0.666 & 0.839 & $\downarrow$ \\
$\mathrm{B}_{0}{ }^{2} / 8 \pi(\mathrm{nPa})$ & 0.145 & 0.110 & 0.142 & 0.146 & 0.98 & 1.33 & $\downarrow$ \\
$\mathrm{J}_{0}\left(\mu \mathrm{Amp} / \mathrm{km}^{2}\right)$ & 2.53 & 1.90 & 2.89 & 2.96 & 1.14 & 1.56 & $\downarrow$ \\
$\Phi_{0}\left(10^{20} \mathrm{Mx}\right)$ & 10.7 & 6.50 & 12.7 & 13.4 & 1.19 & 2.06 & $\downarrow$ \\
\hline
\end{tabular}

* $\sigma$ Median ( $\equiv \sigma \mathrm{Med}$ ) is analogous to $\sigma$, where Median replaces the Mean in the computation.

Table 6B. Parameter variation measured by $\sigma /$ Mean and $\sigma$ Median/Median for $\mathrm{Q}_{0}=1,2$ cases.

\begin{tabular}{lrrrrrrr} 
Quantity & Mean & Median & \multicolumn{1}{c}{$\sigma$} & $\sigma$ Median & $\sigma /$ Mean & $\sigma$ Median/Med & Trend $^{+}$ \\
\hline Diameter $(\mathrm{AU})$ & 0.249 & 0.249 & 0.078 & 0.078 & 0.313 & 0.313 & $\downarrow$ \\
$\mathrm{B}_{\mathrm{o}}(\mathrm{nT})$ & 17.1 & 16.6 & 5.91 & 5.94 & 0.345 & 0.358 & $\downarrow$ \\
$J_{0}\left(\mu \mathrm{Amp} / \mathrm{km}^{2}\right)$ & 1.95 & 1.90 & 0.913 & 0.915 & 0.468 & 0.481 & $\downarrow$ \\
$\mathrm{I}_{\mathrm{T}}\left(10^{8} \mathrm{Amps}\right)$ & 8.37 & 7.28 & 4.47 & 4.60 & 0.534 & 0.632 & $\downarrow$ \\
$\mathrm{B}_{0}{ }^{2} / 8 \pi(\mathrm{nPa})$ & 0.130 & 0.110 & 0.117 & 0.119 & 0.899 & 1.09 & $\downarrow$ \\
$\Phi_{0}\left(10^{20} \mathrm{Mx}\right)$ & 9.21 & 6.70 & 7.24 & 7.67 & 0.786 & 1.15 & $\downarrow$ \\
\hline
\end{tabular}

+ Trend holds for $\sigma$ Med/Med (and approximately for $\sigma /$ Mean).

(MCL) structures. They differ from bona fide MCs from our point of view, because they were not properly fitted by the Lepping et al. (1990) MC model, nor are they likely to be well fitted by any reasonable flux rope model. Their somewhat unusual characteristics may be the result of distant spacecraft passages (with respect to the axes) of actual MCs, but some may be non-MC ejecta. However, all are well worth investigating, especially with respect to possible solar sources, and to see how they compare to ICME lists of events, such as that by Cane and Richardson (2003).

For a summary of "particular properties", we point out that the ratio $\sigma /$ Mean for the derived quantities takes the order (from min to max) as that shown here: $2 \mathrm{R}_{0} \mathrm{~B}_{0}, \mathrm{~J}_{0}$, $\mathrm{I}_{T}, \mathrm{~B}_{0}^{2} / 8 \pi$ (see Table $6 \mathrm{~B}$ for the $\mathrm{Q}_{0}=1,2$ cases). We determined that in $56 \%$ of the total cases WIND MCs drove interplanetary shocks (including a small percentage of upstream pressure pulses), and shocks internal to MCs have been observed about $10 \%$ of the time (Collier et al., 2005). Of our 82 cases there were several MCs that had short durations, with two shortest cases having about $5 \mathrm{~h}$ duration. There were four core-annulus cases (marked by * in the $\mathrm{ck} \% \mathrm{col}$. of Table 1). Occasionally magnetic holes are seen at MC boundaries usually making the identification of the boundaries much easier. The reason for this association is not yet agreed upon. Also it is possible that any specific MC may be part of multiple or complex ejecta (Burlaga et al., 2002; Farrugia and Berdichevsky, 2004). Just as for ICMEs, there is sometimes unusual composition at or near MCs (e.g. Steinberg et al. (1997) where $\mathrm{He}^{++}$solar wind enhancements were observed); also see McComas et al. (1998) on unusual ACE CME composition. Crooker and Intriligator (1996) and Crooker et al. (1998a, b) call attention to the high incidence of MCs occurring at or near sector boundaries.

Other models have been used to fit the (local) defining parameters of MCs with various degrees of success (see Riley et al. (2004) for a review of these and some comparisons of the models' features). Some of these models obtained a better fit of the field intensity in a MC/flux rope than the Lepping et al. (1990) model; for other recent MC/flux rope models see $\mathrm{Hu}$ and Sonnerup (2001, 2002); Hidalgo et al. (2002); Hidalgo (2003); Li et al. (2001); Mulligan et al. (1999); and Vandas et al. (2005a, b). The cylindrical force free flux rope model for MCs has been challenged via comparison with spheroidal models (spherical, oblate, and prolate) by Vandas et al. (1993). In particular, they found that for 14 structures considered, primarily from IMP data, the spheroidal model fits were of comparable accuracy to the cylindrical model fits, but the estimated diameters were generally larger for the spheroidal models. Farrugia et al. (1995) compared the generic flux rope model to spheromak models, in order to ascertain which type gives the more accurate description of actual MCs and found that flux rope models are more consistent with plasma thermodynamic constraints, according to the findings of Osherovich et al. (1993a). 
We plan to modify our MC fitting model to account for internal expansion (e.g. Osherovich et al., 1993b; Berdichevsky et al., 2003), non-circular cross-section (e.g. Lepping et al., 1998), and interaction with the surrounding plasma, and probably in that order. However, we will still view the fundamental MC structure as a distorted (and in some cases severely distorted) cylindrical flux rope, and we plan to continue with a production type of operation, in which any general MC may be processed; this eliminates time consuming tailoring to each case. The results of this new model, as applied to the same WIND data set of 8.6 years, could be compared to the results of the Lepping et al. (1990) model shown here, where it is expected that the field magnitude fit to the observations, at least, would be markedly improved.

\section{Appendix A: Criteria for estimating quality of magnetic cloud fit}

We choose to quantify the quality $\left(\mathrm{Q}_{0}\right)$ of the model parameter-fit of a given magnetic cloud (MC) into three possibilities, $\mathrm{Q}_{0}=1,2,3$, for good, fair, and poor, respectively, given below in terms of magnetic field quantities resulting from use of the MC model (Lepping et al., 1990). However, for the sake of compactness we often refer to Quality as a measure of the MC per se, where it is understood that it is the quality of the MC parameter-fit that is being estimated.

We first describe the characteristics of those MC's that fall into the $\mathrm{Q}_{0}=3$ (poor) category. This category arises from satisfying any one of the following $\mathrm{Q}_{0}=3$ criteria: $\mid$ check $|\geq 55 \%,| \mathrm{CA} \mid \geq 97 \%,\left\langle\mathrm{~B}_{X}>_{\mathrm{Cl}} \leq-1.5 \mathrm{nT}\right.$, either the f-flag or the F-flag=NOT OK, Diameter $\geq 0.45 \mathrm{AU}$, asf $\geq$ $40 \%$, Cone angle $\left(\beta_{C A}\right) \leq 25^{\circ}$ or $\beta_{C A} \geq 155^{\circ}$, and $\chi_{R} \geq$ 0.215. Notice that $\chi_{R}=0.215$ corresponds to a MC field noise level $\mathrm{v}$ of $4.0 \mathrm{nT}$, according to Lepping et al. (2003a, 2004), and this is the highest MC noise level that they found acceptable. The remaining cases, comprising designated " $\mathrm{Q}_{0}=1$ or 2 ", are next examined to differentiate the "good" cases $\left(\mathrm{Q}_{0}=1\right)$ from the "fair" $\left(\mathrm{Q}_{0}=2\right)$ ones. The $\mathrm{Q}_{0}=1$ cases must satisfy all of the following criteria: $\mid$ check $\mid \leq$ $20 \%,\left|<\mathrm{B}_{Y}>_{C l}\right| \leq 3.0 \mathrm{nT}$, asf $\leq 30 \%, 45^{\circ} \leq \beta_{C A} \leq 135^{\circ}$, and $\chi_{R} \leq 0.165$. These are the " $\mathrm{Q}_{0}=1$ set". Notice that $\chi_{\mathrm{R}}=0.165$ corresponds to a MC field noise level $\mathrm{v}$ of $3.0 \mathrm{nT}$, according to Lepping et al. (2003a; 2004). The remaining cases within set 1,2 , i.e. those not satisfying the $\mathrm{Q}_{0}=1$ criteria, are put into category $\mathrm{Q}_{0}=2$.

Obviously there are many ways that a $\mathrm{MC}$ can achieve a $\mathrm{Q}_{0}=3$ quality, so there is no typical $\mathrm{Q}_{0}=3 \mathrm{MC}$. However $\chi_{R}$ and asf are usually the two most important parameters in judging MC quality. The quality criteria (meaning for all $\mathrm{Q}_{0}=1,2,3$ ) were derived from our experience in the application of the Lepping et al. (1990) model and partly from a desire to be consistent with the results of the error study by Lepping et al. (2003a; 2004). Before this formal definition of $\mathrm{Q}_{0}$, MC fitting quality had been assigned somewhat subjectively, but guided mainly by the values of $\chi_{R}$ and asf. These present criteria represent a distinct improvement in assessing quality consistently. It should be stressed that, by our criteria (magnetic field quantities only), a MC may well satisfy the original Burlaga et al. (1981) definition of a MC and still not have good flux rope structure by the Lepping et al. (1990) model, and therefore not qualify for a $\mathrm{Q}_{0}$ of 1 or 2 evaluation.

\section{Appendix B: Notes on the fit-parameter error program}

Estimated errors in MC fit-parameters based on uncertainty due to typical field fluctuation noise within a MC (i.e. source $\# 1$ in Sect. 5.0) for each case are based on the three independent variables $\mathrm{CA}, \beta_{C A}$, and magnetic field (fluctuation) noise level, called $v$ (in $\mathrm{nT}$ ), as assumed by Lepping et al. (2003a); also see the correction described by Lepping et al. (2004). The results of the error analysis presented in Lepping et al. (2003a) provides useful "error-parameter curves", in terms of CA, $\beta_{C A}, v$, given by Figs. 7a, b, c (and see Table 2) in that paper. For practical application some smoothing of the data shown in those figures and described by the interpolation program discussed in Sect. 5.0 here, was necessary. This required linear fits to the curves (with respect to $\mathrm{CA}$ and $\beta_{C A}$ ) and quadratic fits (with respect to $v$ ). This provided a broad range of useful mid-region interpolations for these independent variables. Also, we had to extrapolate to $30^{\circ}$ for the cone angle $\beta_{C A}$ (but now this angle is based on a modification of Eq. (3) (i.e. now $\beta_{C A}=\mid \cos \theta_{A}$ $\left.\cos \phi_{A} \mid\right)$, where the absolute value is applied) before any interpolations were carried out. The extrapolation to $\beta_{C A}=30^{\circ}$ was a simple linear one, because it was based on only two points $\left(\beta_{C A}=60^{\circ}\right.$ and $90^{\circ}$ from Lepping et al., 2003a). Because of the $\beta_{C A}$ angle extrapolation to $30^{\circ}$ and the use of various mirror symmetries in the $\beta_{C A}$-quadrants (by applying the absolute value to Eq. 3), we extend coverage to the five angles $\left(30^{\circ}, 60^{\circ}, 90^{\circ}, 120^{\circ}\right.$, and $\left.150^{\circ}\right)$, before interpolation. It is also important to note that the magnetic field noise level, $v$ (in nT), is obtained from the chi-squared of the MC fit and use of Fig. 6 in Lepping et al. (2003a, 2004). Lepping et al. (2003a) also describe how to make an adjustment to the uncertainty associated with $\mathrm{B}_{0}$, according to a simple proportion with respect to the $\mathrm{B}_{0}$-value arbitrarily chosen in the original statistical study, $\mathrm{B}_{0}$ ( (statistical $)=16.4 \mathrm{nT}$; i.e. corrected- $\sigma_{B_{0}}=\sigma_{B_{0}} \times$ actual $\mathrm{B}_{0} / 16.4 \mathrm{nT}$. Final remark: notice that $\beta_{C A}$ is equivalent to $\mathrm{PhiE}$ (the estimated axial longitude, shown for $60^{\circ}$ and $90^{\circ}$ ) in the main Table 1 and Figs. $7 \mathrm{a}, \mathrm{b}$, c of Lepping et al. (2003a).

Acknowledgements. We thank the WIND/MFI and SWE teams, and in particular, K. Ogilvie, the SWE principal investigator, for the care they employ in producing the plasma and field data used in part of this work. DBB and CCW acknowledge NSF support under Space Weather Grant ATM 0208414. CCW is supported partially by a NASA Living with a Star Program under NASA Grants number NAG-12527 (with RPL) and NAG-512467. DBB thanks NASA for support under Grant number NAG-02035.

Topical Editor T. Pulkkinen thanks M. Vandas and another referee for their help in evaluating this paper. 


\section{References}

Antiochos, S. K. and DeVore, C. R.: The role of magnetic reconnection in solar activity, in: Sun- Earth Plasma Connections, Geophys. Monogr. Ser., edited by: Burch, J. L., Carovillano, R. L., and Antiochos, S. K, AGU, Washington, D.C., Vol. 109, 113120, 1999.

Barnden, L. R.: The large scale magnetic configuration associated with Forbush decreases, in: Proceedings of the Conference on Cosmic Plasma Physics, held at ESRIN, Frascati, Italy, 20-24 September, 1971, edited by: Schindler, K., Plenum Press, New York, 351-358, 1972.

Baumgartel, K., Sauer, K., and Dubinin, E.: Towards understanding magnetic holes: Hybrid simulations, J. Geophys. Res., SSC-4, doi:10.1029/2003GL017373, 2003.

Berdichevsky, D., Richardson, I., Thompson, B. J., Reames, D., MacDowall, R., Plunkett, S. P., Michels, D. J., Kaiser, M. L., Lepping, R. P., Ogilvie, K. W., and Stone, R. G.: Examples of fast solar wind transients, their sources and the forecast of possible geomagnetic impact, Geofisica Interacional, 39, 1, 5-11, 2000.

Berdichevsky, D. B., Farrugia, C. J., Thompson, B. J., Lepping, R. P., Reames, D. V., Kaiser, M. L., Steinberg, J. T., Plunkett, S. P., and Michels, D. J.: Halo-Coronal mass ejections near the 23rd solar minimum: Lift-off at the Sun, interplanetary tracking, and in-situ observations at 1 AU, Ann. Geophys., 20, 891-916, 2002, SRef-ID: 1432-0576/ag/2002-20-891.

Berdichevsky, D. B., Lepping, R. P., and Farrugia, C. J.: Geometric considerations of the evolution of magnetic flux ropes, Phys. Rev., E 67, 036405, 1-8, 2003.

Bothmer, V.: Sources of magnetic helicity over the solar cycle, in: Solar variability as an input to the Earth's environment, International Solar Cycle Studies (ISCS) Symposium, 23-28 June 2003, Tatranská Lomnica, Slovak Republic, edited by: Wilson, A., ESA SP-535, Noordwijk: ESA Publications Division, ISBN 92-9092-845-X, 419-428, 2003.

Bothmer, V. and Rust, D. M.: The field configuration of magnetic clouds and the solar cycle, in: Coronal Mass Ejections, Geophys. Monogr. Ser., Vol. 99, edited by: Crooker, N., Joselyn, A., and Feynman, J., AGU, Washington, D.C., 139-146, 1997.

Bothmer, V. and Schwenn, R.: Magnetic cloud observations by the Helios spacecraft, Solar Wind Seven, edited by: Marsch, E. and Schwenn, R., Pergamon Press, Oxford, England, 599-602, 1992.

Bothmer, V. and Schwenn, R.: Eruptive prominences as sources of magnetic clouds in the solar wind, Proceedings of the II Soho Workshop at Elba, Italy, 1993, Space Sci. Rev., 70, 215-220, 1994.

Bothmer, V. and Schwenn, R.: The structure of magnetic clouds in the solar wind, Ann. Geophys., 16, 1-24, 1998,

\section{SRef-ID: 1432-0576/ag/1998-16-1.}

Burlaga, L. F.: Magnetic clouds: Constant alpha forcefree configurations, J. Geophys. Res., 93, 7217-7224, 1988.

Burlaga, L. F.: Interplanetary Magnetohydrodynamics, Oxford Univ. Press, New York, 89-114, 1995.

Burlaga, L. F., Sittler Jr., E. C., Mariani, F., and Schwenn, R.: Magnetic loop behind an inter- planetary shock: Voyager, Helios, and IMP-8 observations, J. Geophys. Res., 86, 6673-6684, 1981.

Burlaga, L. F., Behannon, K. W., and Klein, L .W.: Compound streams, magnetic clouds, and major geomagnetic storms, J. Geophys. Res, 92, 5725-5734, 1987.

Burlaga, L. F., Lepping, R. P., and Jones, J. A.: Global configuration of a magnetic cloud, in: Physics of Magnetic Flux Ropes,
Geophys. Monogr. Ser., edited by: Russell, C. T., Priest, E. R., and Lee, L. C., AGU, Washington, D.C., Vol. 58, 373-377, 1990.

Burlaga, L. F., Fitzenreiter, R. J., Lepping, R. P., Ogilvie, K. W., Szabo, A., Lazarus, A. J., Steinberg, J., Gloeckler, G., Howard, R. A., Michels, D. J., Farrugia, C. J., Lin, R. P., and Larson, D. E.: A magnetic cloud containing prominence material: January 1997, J. Geophys. Res., 103, 277-286, 1998.

Burlaga, L. F., Ness, N. F., Richardson, J. D., and Lepping, R. P.: The Bastille Day Shock and Merged Interaction Region at 63 AU: Voyager 2 Observations, Solar Phys., 204, 399-410, 2001.

Burlaga, L. F., Plunkett, S., and St. Cyr, C.: Successive CMEs and complex ejecta, J. Geophys. Res., 107, 1266-1277, 2002.

Cane, H. V. and Richardson, I. G.: Interplanetary coronal mass ejections in the near-Earth solar wind during 1996-2002, J. Geophys. Res., 108(A4), 1156, doi:10.1029/2002JA009817, 2003.

Cane, H. V., Richardson, I. G., and von Rosenvinge, T. T.: Cosmic ray decreases: 1964-1994, J. Geophys. Res., 101, 21 561$21572,1996$.

Chen, J.: Theory of prominence eruption and propagation: Interplanetary consequences, J. Geophys. Res., 101, 27 499-27 520, 1996.

Chen, J., Cargill, P. J., and Palmadesso, P. J.: Predicting solar wind structures and their geoeffectiveness, J. Geophys. Res., 102, 14 701-14 720, 1997.

Collier, M. R., Szabo, A., Farrell, W., Slavin, J. A., Lepping, R. P., Hamilton D. C., Gloeckler, G., Ho, G., Bochsler, P., Larson, and Ofman, D. L.: Reconnection remnants in the magnetic cloud of 18-19 October, 1995: A shock, monochromatic wave, heat flux drop out, and energetic ion beam, J. Geophys. Res., 106, 15985 $16000,2001$.

Collier, M. R., Lepping, R. P., and Berdichevsky, D.: A statistical study of interplanetary shocks and pressure pulses internal to magnetic clouds, NASA-GSFC internal document, 2005.

Crooker, N. U. and Intriligator, D. S.: A magnetic cloud as a distended flux-rope occlusion in the heliospheric current sheet, J. Geophys. Res., 101, 24 343-24348, 1996.

Crooker, N. U., Gosling, J. T., and Kahler, S. W.: Magnetic clouds at sector boundaries, J. Geophys. Res., 103, 301-306, 1998a.

Crooker, N. U., McAllister, A. H., Fitzenreiter, R. J., Linker, J. A., Larson, D. E., Lepping, R. P., Szabo, A., Steinberg, J. T., Lazarus, A. J., Mikic, Z., and Lin, R. P.: Sector boundary transformation by an open magnetic cloud, J. Geophys. Res., 103, 26859-26868, 1998b.

Farrugia, C. J.: Recent work on modeling the global field line topology of interplanetary magnetic clouds, Geophys. Monogr. Ser., 99, edited by: Crooker, N., Joselyn, J., and Feynman, J., AGU, Washington D.C., 177-188, 1997.

Farrugia, C. J. and Berdichevsky, D. B.: Evolutionary signatures in complex ejecta and their driven shocks, Ann. Geophys., 22, 3679-3698, 2004,

SRef-ID: 1432-0576/ag/2004-22-3679.

Farrugia, C. J., Burlaga, L. F., Freeman, P., Lepping, R. P., and Osherovich, V.: A comparative study of expanding force-free constant alpha magnetic configurations with application to magnetic clouds, in: Solar Wind Seven, edited by: Marsch, E. and Schwenn, R., Pergamon New York, 611-614, 1992.

Farrugia, C. J., Osherovich, V. A., and Burlaga, L. F: The magnetic flux rope versus the spheromak as models for interplanetary magnetic clouds, J. Geophys. Res, 100, 12 293-12 306, 1995.

Farrugia, C. J., Burlaga, L. F., and Lepping, R. P.: Magnetic Clouds and the Quiet-storm Effect at Earth, in: Magnetic Storms, Geo- 
phys. Monogr. Ser., 98, edited by: Tsurutani, B. T., Gonzales, W. D., and Kamide, Y., AGU, Washington, D.C., 91-106, 1997.

Farrugia, C. J., Vasquez, B. J., Torbert, R. B., Janoo, L., Richardson, I. G., Reiner, L. M., Berdichevsky, D., Burlaga, L. F., Ogilvie, K. W., Osherovich, V. A., Fitzenreiter, R. J., Szabo, A., and Lazarus, A. J.: Multi-instrument study of the December 1996 magnetic cloud and associated interplanetary disturbances, in: The Solar Wind - Magnetosphere System-3, 1999, edited by: Biernart, H. K., Farrugia, C. J., Vogl, D., Austrian Academy of Sciences, Austria, 99-109, 2000.

Gopalswamy, N., Hanaoka, Y., Kosugi, T., Lepping, R. P., Steinberg, J. T., Plunkett, S., Howard, R. A., Thompson, B. J., Gurman. J., Ho, G., Nitta, N., and Hudson, H. S.: On the relationship between coronal mass ejections and magnetic clouds, Geophys. Res. Lett., 25, 2485-2488, 1998.

Gopalswamy, N., Lara, A., Lepping, R. P., Kaiser, M. L., Berdichevsky, D., and St. Cyr, O. C.: Interplanetary acceleration of coronal mass ejections, Geophys. Res. Lett., 27, 145-148, 2000.

Goldstein, H.: On the field configuration in magnetic clouds, in: Solar Wind Five, edited by: Neugebauer, M., NASA Conf. Publ., 2280, 731-733, 1983.

Goldstein, R., Neugebauer, M., and Clay, D.: A statistical study of CME plasma flows, J. Geophys. Res., 103, 4761-4766, 1998.

Gosling, J. T.: Coronal mass ejections and magnetic flux ropes in interplanetary space, in: Physics of Magnetic Flux Ropes, Geophys. Monogr. Ser., 58, edited by: Russell, C. T., Priest, E. R., and Lee, L. C., AGU, Washington, D.C., 343-364, 1990.

Gosling, J. T.: Coronal mass ejections: an overview, in: coronal Mass Ejections, Geophys. Monogr. Ser., Vol. 99, edited by: Crooker, N., Joselyn, J., and Feynman, J., AGU, Washington D. C., 9-16, 1997.

Gosling, J. T.: Coronal mass ejections, in: The 26th International Cosmic Ray Conference, edited by: Dingus, B. L., Kieda, D., and Salamon, M., AIP Conf. Proc., 561, 59-79, 2000.

Gosling, J. T., Pizzo, V., and Bame, S. J.: Anomalously low proton temperatures in the solar wind following interplanetary shock waves: Evidence for magnetic bottles?, J. Geophys. Res., 78, 2001-2009, 1973.

Hidalgo, M. A., Cid, C., Vinas, A. F., and Sequeiros, J.: A non-force-free approach to the topology of magnetic clouds in the solar wind, J. Geophys. Res, 107 (A1), doi:10.1029/2001JA900100, 2002.

Hidalgo, M. A.: A study of the expansion and distortion of the cross section of magnetic clouds in the interplanetary medium, J. Geophys. Res, 108 (A8), doi:10.1029/2002JA009818, 2003.

Hirshberg, J., Bame, S., and Robbins, D. E.: Solar flares and solar helium enrichments: July 1965-1967, Solar Phys., 23, 467-486, 1972.

$\mathrm{Hu}$, Q. and Sonnerup, B. U. O.: Reconstruction of magnetic flux ropes in the solar wind, Geophys. Res. Lett., 28(3), 467-470, 2001.

$\mathrm{Hu}$, Q. and Sonnerup, B. U. O.: Reconstruction of magnetic flux ropes in the solar wind: Orientations and configurations, J. Geophys. Res, 107 (A7), doi:10.1029/2001JA000293, 2002.

Kahler, S.: Coronal mass ejections, Rev. of Geophys., 25, 663-675, 1987.

Kamide, Y., Mc Pherron, R. L., Gonzalez, W. D., et al.: Magnetic storms: Current understanding and outstanding questions, in: Magnetic Storms, Geophys. Monogr. Ser., 98, edited by: Tsurutani, B. T., Gonzalez, W. D., Kamide, Y., and Arballo, J. K., AGU, Washington D.C., 1-19, 1997.
Klein, L. and Burlaga, L. F.: Interplanetary magnetic clouds at 1 AU, J. Geophys. Res., 87, 613-624, 1982.

Kumar, A. and Rust, D. M.: Interplanetary magnetic clouds, helicity conservation, and current-core flux ropes, J. Geophys. Res., 101, $15667-15684,1996$.

Larson, D. E, Lin, R. P., McTiernan, J., McFadden, J. P., Ergun, R. E., McCarthy, M., Rème, H., Sanderson, T. R., Kaiser, M., Lepping, R. P., and Mazur, J.: Tracing the topology of the 1820 October 1995, magnetic cloud with $\sim 0.1-10^{2} \mathrm{keV}$ electrons, Geophys. Res. Lett., 24, 1911-1914, 1997.

Lepping, R. P. and Berdichevsky, D.: Interplanetary magnetic clouds: Sources, properties, modeling, and geomagnetic relationship, Research Signpost, Recent Res. Devel. Geophysics, 3, 77-96, 2000

Lepping, R. P., Jones, J. A., and Burlaga, L. F. : Magnetic field structure of interplanetary magnetic clouds at $1 \mathrm{AU}, \mathrm{J}$. Geophys. Res., 95, 11 957-11 965, 1990.

Lepping, R. P., Acuña, M. H., and Burlage, L. F.: The WIND magnetic field investigation, The Global Geospace Mission, Space Sci. Rev. 71, 207-229, 1995.

Lepping, R. P., Burlaga, L. F., Szabo, A., Ogilvie, K. W., Mish, W. H., Vassiliadis, D., Lazarus, A. J., Steinberg, J. T., Farrugia, C. J., Janoo, L., and Mariani, F.: The Wind magnetic cloud and events of 18-20 October, 1995: Interplanetary properties and as triggers for geomagnetic activity, J. Geophys. Res., 102, 14 049-14063, 1997a.

Lepping, R. P., Szabo, A., DeForest, C. E., and Thompson, B. J.: Magnetic flux in modeled magnetic clouds at $1 \mathrm{AU}$ and some specific comparisons to associated photospheric flux, Proceedings of the 31st ESLAB Symposium on Correlated Phenomena at the Sun, in the Heliosphere, and in Geospace, ESA SP-415, 163-170, 1997b.

Lepping, R. P., Berdichevsky, D., Szabo, A., Goodman, M., and Jones, J.: Modification of magnetic cloud model: Elliptical cross-section, AGU EOS Transactions (SH11A-05), 79, F696, 1998.

Lepping, R. P., Berdichevsky, D. B., Burlaga, L. F., Lazarus, A. J., Kasper, J., Desch, M. D, Wu, C.-C., Reames, D. V., Singer, H., Smith, C., and Ackerson, K.: The Bastille Day magnetic clouds and upstream shocks at earth: Interplanetary observations, Solar Phys., 204, 287-305, 2001.

Lepping, R. P., Berdichevsky, D., Szabo, A., Lazarus A. J., and Thompson, B. J.: Upstream shocks and interplanetary magnetic cloud speed and expansion: SUN, WIND and Earth observations, Space Weather Study using Multipoint Techniques,edited by: Lyu, L.-H., Proceedings of the COSPAR Colloquium in Pacific Green Bay, Taiwan, 27-29 September 2000, Pergamon Press, 87-96, 2002.

Lepping, R. P., Berdichevsky, D., and Ferguson, T.: Estimated Errors in Magnetic Cloud Model Fit-Parameters with Force Free Cylindrically Symmetric Assumptions, J. Geophys. Res., 108(A10), 1356, doi:10.1029/2002JA009657, 2003a.

Lepping, R. P., Berdichevsky, D. B., and Wu, C.-C.: Sun-Earth electrodynamics: The solar wind connection, Recent Res. Devel. Astrophys., Research Signpost, 1, ISBN: 81-271-0004-8, 139-171, 2003b.

Lepping, R. P., Berdichevsky, D. B., Szabo, A., Arqueros, C., and Lazarus, A. J.: Profile of a generic magnetic cloud at $1 \mathrm{AU}$ for the quiet solar phase: WIND observations: Solar Phys., 212, 425444, 2003c.

Lepping, R. P., Berdichevsky, D., and Ferguson, T.: Correction (to paper: Estimated errors in magnetic cloud model fit- 
parameters with force free cylindrically symmetric assumptions by the same authors), J. Geophys. Res., 109, A07101, doi:10.1029/2004JA010517, 2004.

Lepping, R. P., Wu, C.-C., and Berdichevsky, D. B.: Automated identification of magnetic clouds and cloud-like regions at $1 \mathrm{AU}$ : Occurrence rate and type categorization, Ann. Geophys., 23, 2687-2704, 2005,

SRef-ID: 1432-0576/ag/2005-23-2687.

Li, Y., Luhmann, J. G., Mulligan, T., Hoeksema, J. T., Arge, C. N., Plunkett, S. P., and St. Cyr, O. C.: Earthward directed CMEs seen in large-scale coronal magnetic field changes, SOHO LASCO coronagraph and solar wind, J. Geophys. Res., 106, 25 103$25120,2001$.

Lin, R. P., Anderson, K. A., Ashford, S., Carlson, C. W., et al.: A three-dimensional plasma and energetic particle investigation for the WIND spacecraft, Space Sci. Rev., 71, 125-153, 1995.

Lundquist, S.: Magnetohydrostatic fields, Ark. Fys., 2, 361-365, 1950.

Martin, S. F. and McAllister, A. H.: Predicting the sign of magnetic helicity in erupting filaments and coronal mass ejections, in: Coronal Mass Ejections, Geophys. Monogr. Ser., Vol. 99, edited by: Crooker, N., Joselyn, J., and Feynman, J., AGU, Washington D.C., 127-138, 1997.

Marubashi, K.: Structure of the interplanetary magnetic clouds and their solar origins, Adv. Space Res., 6(6), 335-338, 1986.

Marubashi, K.: Interplanetary magnetic flux ropes and solar filaments, in: Coronal Mass Ejections, Geophys. Monogr. Ser., Vol. 99, edited by: Crooker, N., Joselyn, J., and Feynman, J., AGU, Washington D.C., 147-156, 1997.

McComas, D. J., Bame, S. J., Barker, P. L., et al.: An unusual coronal mass ejection: First Solar Wind Electron, Proton, Alpha Monitor (SWEPAM) results from the Advanced Composition Explorer, Geophys. Res. Lett., 25, 4289-4292, 1998.

Moldwin, M. B., Ford, S., Lepping, R. P., Slavin, J. A., and Szabo, A.: Small-scale magnetic flux ropes in the solar wind, Geophys. Res. Lett., 27(\#1), 57-60, 1999.

Mulligan, T., Russell, C. T., and Luhmann, J. G.: Solar cycle evolution of the structure of magnetic clouds in the inner heliosphere, Geophys. Res. Lett., 25, 2959-2962, 1998.

Mulligan, T., Russell, C. T., Anderson, G. J., Lohr, D. A., Toth, B. A., Zanetti, L. J., Acuna, M. H., Lepping, R. P., Gosling, J. T., and Luhmann, J. G.: Flux rope modeling of multiple spacecraft observations of interplanetary coronal mass ejections over variable separation distances, SH42A-02, abstract, EOS Transactions, AGU 1999 Spring meeting, S265, 1999.

Mulligan, T., Russell, C. T., Anderson, G. J., and Acuña, M. H.: Multiple spacecraft flux rope modeling of the Bastille Day magnetic cloud, Geophys. Res. Lett., 29,(23), 4417-4420, 2001.

Ogilvie, K. W., Chorney, D.-J., Fitzenreiter, R. J., et al.: SWE, A comprehensive plasma instrument for the WIND spacecraft, The Global Geospace Mission, Space Sci. Rev., 71, 55-77, 1995.

Osherovich, V. A. and Burlaga, L. F.: Magnetic clouds, in: Coronal Mass Ejections, Geophys. Monogr. Ser., 99, edited by: Crooker, N., Joselyn, J., and Feynman, J., AGU, Washington D.C., 157$168,1997$.

Osherovich, V. I., Farrugia, C. J., Burlaga, L. F., Lepping, R. P., Fainberg, J., and Stone, R. G.: Polytropic relationship for interplanetary magnetic clouds, J. Geophys. Res., 98, 15 331-15342, 1993a.

Osherovich, V. I., Farrugia, C. J., and Burlaga, L. F.: Dynamics of aging magnetic clouds, Adv. Space Res., 13(6), 57-62, 1993b.

Priest, E.: The equilibrium of magnetic flux ropes (Tutorial lecture), in: Physics of Magnetic Flux Ropes, Geophys. Monogr. Ser., Vol. 58, edited by: Russell, C. T., Priest, E. R., and Lee, L. C., AGU, Washington D.C., 1-22, 1990.

Richardson, I. G. and Cane, H.: The fraction of interplanetary coronal mass ejections that are in magnetic clouds: Evidence for a solar cycle variation, Geophys. Res. Lett., 31, L18804, doi:10.1029/2004GL020958, 2006.

Riley, P., Linker, J. A., Lionello, R., Mikic, Z., Odstrcil, D., Hidalgo, M. A., Cid, C., Hu, Q., Lepping, R. P., Lynch, B. J., and Rees, A.: Fitting Flux Ropes to a Global MHD Solution: A Comparison of Techniques, J. Atmos. S.-P., 66, 15-16, 1321-1331, 2004.

Rust, D. M.: Spawning and shedding of helical magnetic fields in the solar atmosphere: Geophys. Res. Lett., 21, 241-244, 1994.

Rust, D. M.: The Solar Stereo Mission, in: Sun-Earth Plasma Connections, Geophys. Monogr. Ser., 109, edited by: Burch, J. L., Carovillano, R. L., and Antiochos, S. K., AGU, Washington D.C., 213-224, 1999.

Rust, D. M and Kumar, A.: Helical magnetic fields in filaments, Solar Phys., 155, 69-98, 1994.

Schwenn, R.: Mass ejections from the sun and their interplanetary counterparts, in Solar Wind Eight, edited by: Winterhalter, D., Gosling, J. T., Habbal, S. R., Kurth, W. S., and Neugebauer, M., AIP Press, Conference Proceeding 328, Woodbury, N.Y., 426429, 1996.

Shimazu, H. and Marubashi, K.: New method of detecting interplanetary flux ropes, J. Geophys. Res., 105, 2365-2373, 2000.

Skoug, R. M., Feldman, W. C., Gosling, J. T., McComas, D. J., Reisenfeld, D. B., Smith, C. W., Lepping, R. P., and Balogh, A.: Radial variation of solar wind electrons inside a magnetic cloud observed at 1 and 5 AU, J. Geophys. Res., 105, 27 269-27 275, 2000.

Steinberg, J. T., Lazarus, A. J., Ogilvie, K. W., Fitzenreiter, R. J., Lepping, R. P., and Szabo, A.: $\mathrm{He}^{++}$enhancements in the solar wind during large transient events as seen from the wind spacecraft, Supplement to AGU, EOS Transactions, 78, 46, F539 (SH22A-01), 1997.

Tandberg-Hanssen, E.: The nature of solar prominences, Kluwer Academic Pub., Dordrecht, Holland, 1995.

Tsurutani, B. T. and Gonzalez, W. D.: The interplanetary causes of magnetic storms: A review, in: Magnetic Storms, Geophys. Monogr. Ser., 98, edited by: Tsurutani, B. T., Gonzalez, W. D., Kamide, Y., and Arballo, J. K., AGU, Washington D.C., 77-89, 1997.

Vandas, M, Fischer, S., Pelant, P., and Geranios, A.: Spheroidal models of magnetic clouds and their comparison with spacecraft measurements, J. Geophys. Res., 98, 11 467-11 475, 1993.

Vandas, M., Romashets, E. P., and Watari, S.: Magnetic clouds of oblate shapes, Planet Space Sci., 53, 19- 24, 2005a.

Vandas, M., Romashets, E. P., Watari, S., Geranios, A., Antoniadou, E., and Zacharopoulou, O.: Comparison of force-free flux rope models with observations of magnetic clouds, Adv. Space Res., in press, 2005b.

Vrsňak, B., Ruždjak, V., and Rompolt, B.: Stability of prominences exposing helical-like patterns, Solar Phys., 136, 151-167, 1991.

Webb, D. F., Lepping, R. P., Burlaga, L. F., DeForest, C. E., Larson, D. E., Martin, S. F., Plunkett, S. P., and Rust, D. M.: The origin and development of the May 1997 magnetic cloud, J. Geophys. Res., 105, 27 251-27 259, 2000.

Webb, D. F., Crooker, N. U., Plunkett, S. P., and St. Cyr, O. C.: The solar sources of geoeffective structures, in: Space Weather, Geophys. Monogr. Ser., Vol. 125, edited by: Song, P., Singer, 
H. J., and Siscoe, G. L., AGU, Washington D.C., 123-141, 2001. Wilson, R. M.: On the behavior of the $D_{s t}$ geomagnetic index in the vicinity of magnetic cloud passages at Earth, J. Geophys. Res., 95, 215-219, 1990.

Wu, C.-C. and Lepping, R. P.: The effects of magnetic clouds on the occurrences of geomagnetic storms: The first four years of WIND, J. Geophys. Res., 107 (A10), 1314-1321, doi:10.1029/2001JA000,161, 2002a.

Wu, C.-C. and Lepping, R. P.: Effect of Solar Wind Velocity on Magnetic Cloud-Associated Magnetic Storm Intensity, J. Geophys. Res., 107(A11), 1346, doi:10.1029/2002JA009396, 2002b.
Wu, C.-C. and Lepping, R. P. : Relationships for predicting magnetic cloud related geomagnetic storm intensity: J. Atmos. SolarTerr. Phys., 67, 283-291, doi:10.1016/2004.07.040, 2005.

Wu, C.-C., Liou, K., Lepping, R. P., and Meng, C.-I.: Identification of substorms within storms, J. Atmos. Solar-Terr. Phys., 66, 125132, 2004.

Zurbuchen, T. and Richardson, I. G.: In situ solar wind and magnetic field signatures of interplanetary coronal mass ejections, Space Sci. Rev., in press, 2006. 